\title{
Synthesis and Characterization of Zirconium and Iron Complexes Containing Substituted Indenyl Ligands: Evaluation of Steric and Electronic Parameters.
}

Christopher A. Bradley, Samuel Flores-Torres, Emil Lobkovsky and Paul J. Chirik*

Department of Chemistry and Chemical Biology

Baker Laboratory, Cornell University Ithaca, NY 14853 


\section{Table of Contents}

Experimental procedures for the preparation of substituted indenyl ligands. $\quad$ S3

Atom labeling scheme and X-ray crystallographic data for $\mathbf{1 - \mathbf { C l } _ { \mathbf { 2 } }} \quad$ S7

Atom labeling scheme and X-ray crystallographic data for $\mathbf{2 - \mathbf { C l } _ { 2 }} \quad \mathrm{S} 15$

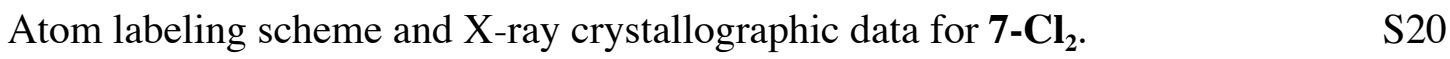

Atom labeling scheme and X-ray crystallographic data for $\mathbf{9 -} \mathbf{C l}_{\mathbf{2}} . \quad$ S26

Atom labeling scheme and X-ray crystallographic data for 3-(CO) $)_{2} \quad$ S31

$\begin{array}{ll}\text { Atom labeling scheme and X-ray crystallographic data for } \mathbf{1 3} & \text { S37 }\end{array}$

Atom labeling scheme and X-ray crystallographic data for rac-17 $\quad$ S41

Representative IR spectra recorded in a $\mathrm{KBr}$ pellet $\quad$ S45

Eyring plot for $\mathbf{1 3}$ from variable temperature NMR data recorded in toluene- $d_{8} . \quad$ S46

${ }^{1} \mathrm{H}$ NMR spectrum of $\mathrm{rac} / \mathrm{meso} \mathbf{- 1 7}$ in toluene- $d_{8}$ as a function of temperature. $\quad \mathrm{S} 47$

Low temperature ${ }^{1} \mathrm{H}$ NMR spectra of $\mathbf{1 4} \quad$ S48 
Preparation of $\mathrm{Li}\left[\mathrm{C}_{9} \mathrm{H}_{6}-\left(\mathrm{SiMe}_{2} \mathrm{Ph}\right)\right]$. A $1 \mathrm{~L}$ round-bottomed flask was charged with $10.10 \mathrm{~g}$ (40.3 mmol) of $\mathrm{C}_{9} \mathrm{H}_{7}-\left(\mathrm{SiMe}_{2} \mathrm{Ph}\right)$ and the resulting solution chilled in the cold well for 30 minutes. To the light yellow solution, $28.0 \mathrm{~mL}$ (44.8 mmol) of $1.6 \mathrm{M}{ }^{n} \mathrm{BuLi}$ was added and the reaction was stirred overnight depositing a white solid. The resulting lithium salt was collected by filtration on a medium porosity frit yielding $9.39 \mathrm{~g}(91 \%)$ of $\mathrm{Li}\left[\mathrm{C}_{9} \mathrm{H}_{6}-\left(\mathrm{SiMe}_{2} \mathrm{Ph}\right)\right]$.

Preparation of $\mathbf{C}_{9} \mathbf{H}_{6}-\mathbf{1 , 3}-\left(\mathrm{SiMe}_{2} \mathrm{Ph}\right)_{2}$. A $500 \mathrm{~mL}$ round bottomed flask was charged with $9.39 \mathrm{~g}(36.6 \mathrm{mmol})$ of $\mathrm{Li}\left[\mathrm{C}_{9} \mathrm{H}_{6}-\left(\mathrm{SiMe}_{2} \mathrm{Ph}\right)\right]$ and dissolved in THF. The resulting solution was chilled in a liquid nitrogen cooled cold well for 30 minutes, and $6.1 \mathrm{~mL}$ (36.6 mmol) of $\mathrm{SiMe}_{2} \mathrm{PhCl}$ was added. The reaction mixture was stirred overnight and the solvent removed in vacuo. The resulting yellow oil was extracted into pentane and filtered through Celite. Subsequent solvent removal afforded $11.76 \mathrm{~g}(84 \%)$ of a yellow oil identified as predominantly one isomer of $\mathrm{C}_{9} \mathrm{H}_{6}-1,3-\left(\mathrm{SiMe}_{2} \mathrm{Ph}\right)_{2} .{ }^{1} \mathrm{H}$ NMR (benzene- $\left.d_{6}\right)$ : $\square$

$=0.0\left(\mathrm{~s}, 3 \mathrm{H}, \mathrm{Si} M e_{2} \mathrm{Ph}\right), 0.05(\mathrm{~s}, 3 \mathrm{H}, \mathrm{SiMe} 2 \mathrm{Ph}), 0.46\left(\mathrm{~s}, 3 \mathrm{H}, \mathrm{Si} M e_{2} \mathrm{Ph}\right), 0.47(\mathrm{~s}, 3 \mathrm{H}$, $\mathrm{Si}_{2} \mathrm{Ph}$ ), 3.65 (d, $\left.2 \mathrm{~Hz}, 1 \mathrm{H}, \mathrm{Cp}\right), 6.83$ (d, $2 \mathrm{~Hz}, 1 \mathrm{H}, \mathrm{Cp}$ ), 7.05-7.18 (br m, 6H, Ind/Ph), 7.24 (m, 2H, Ind/Ph), 7.30 (m, 2H, Ind/Ph), 7.44 (m, 2H, Ind/Ph), 7.51 (m, 2H, Ind/Ph). ${ }^{13} \mathrm{C}$ NMR (benzene- $\left.d_{6}\right): \square=-4.76\left(\mathrm{Si} e_{2} \mathrm{Ph}\right),-4.16\left(\mathrm{Si} M e_{2} \mathrm{Ph}\right),-1.99\left(\mathrm{Si} e_{2} \mathrm{Ph}\right),-1.88$ $\left(\mathrm{Si} M e_{2} \mathrm{Ph}\right), 48.45,122.92,123.27$ (Cp), 123.90, 125.35, 127.61, 127.95, 129.32, 129.57, 131.20, 134.76, 137.16, 138.69, 140.24, 146.17, 147.49, 147.89 (Ind/Ph). 
Preparation of $\mathrm{Li}\left[\mathrm{C}_{9} \mathrm{H}_{5}-\mathbf{1 , 3}-\left(\mathrm{SiMe}_{2} \mathrm{Ph}\right)_{2}\right]$. This molecule was prepared in an identical manner to $\mathrm{Li}\left[\mathrm{C}_{9} \mathrm{H}_{6}-1-\left(\mathrm{SiMe}_{2} \mathrm{Ph}\right)\right]$ using $11.76 \mathrm{~g}(30.6 \mathrm{mmol})$ of $\mathrm{C}_{9} \mathrm{H}_{6}-1,3-\left(\mathrm{SiMe}_{2} \mathrm{Ph}\right)_{2}$ and $21.0 \mathrm{~mL}(33.6 \mathrm{mmol})$ of $1.6 \mathrm{M}$ "BuLi yielding $6.50 \mathrm{~g}(54 \%)$ of $\mathrm{Li}^{\mathrm{n}}\left[\mathrm{C}_{9} \mathrm{H}_{5}-1,3-\left(\mathrm{SiMe}_{2} \mathrm{Ph}\right)_{2}\right]$ as an off-white solid.

Preparation of $\mathbf{C}_{9} \mathbf{H}_{7}-\left(\mathrm{SiMe}_{2}{ }^{\mathrm{t}} \mathrm{Bu}\right)$. This molecule was prepared in an identical manner to $\mathrm{C}_{9} \mathrm{H}_{6}-1,3-\left(\mathrm{SiMe}_{2} \mathrm{Ph}\right)_{2}$ with $10.54 \mathrm{~g}(86.3 \mathrm{mmol})$ of $\mathrm{Li}\left[\mathrm{C}_{9} \mathrm{H}_{7}\right]$ and $13.01 \mathrm{~g}(86.3 \mathrm{mmol})$ of $\mathrm{SiMe}_{2}{ }^{\mathrm{t}} \mathrm{BuCl}$. Subsequent workup afforded $17.2 \mathrm{~g}(84 \%)$ of a yellow liquid identified as multiple isomers of $\mathrm{C}_{9} \mathrm{H}_{7}-\left(\mathrm{SiMe}_{2}{ }^{\mathrm{t}} \mathrm{Bu}\right.$ ). ${ }^{1} \mathrm{H}$ NMR (benzene- $d_{6}$ ): (Major isomer) $\square=0.37$ (s, 3H, SiMe ${ }_{2}{ }^{\mathrm{t}} \mathrm{Bu}$ ), 0.80 (s, 3H, SiMe ${ }_{2}{ }^{\mathrm{t}} \mathrm{Bu}$ ), 0.86 (s, 9H, SiMe ${ }_{2}{ }^{\mathrm{t}} \mathrm{Bu}$ ), 3.57 (br s, 1H, Cp), 7.06 (br s, 1H, Cp), 7.20 (t, $10 \mathrm{~Hz}, 1 \mathrm{H}$, Ind), 7.30 (t, $10 \mathrm{~Hz}, 1 \mathrm{H}$, Ind), 7.50 (d, $10 \mathrm{~Hz}, 1 \mathrm{H}$, Ind), $7.76\left(\mathrm{~d}, 10 \mathrm{~Hz}, 1 \mathrm{H}\right.$, Ind). (Minor Isomer) $\mathrm{Z}=-0.41$ (s, 3H, SiMe ${ }_{2}{ }^{\mathrm{B}} \mathrm{Bu}$ ), $0.10(\mathrm{~s}, 3 \mathrm{H}$, $\mathrm{Si} e_{2}{ }^{\mathrm{t}} \mathrm{Bu}$ ), 1.07 (s, 9H, $\mathrm{Si} M e_{2}{ }^{\mathrm{t}} \mathrm{Bu}$ ), 3.14 (br s, 1H, Cp), 7.06 (br s, $\left.1 \mathrm{H}, \mathrm{Cp}\right), 7.18(\mathrm{~m}, 1 \mathrm{H}$, Ind), $7.46(\mathrm{~d}, 10 \mathrm{~Hz}, 1 \mathrm{H}$, Ind), 7.62 (d, $10 \mathrm{~Hz}, 1 \mathrm{H}$, Ind). One Ind resonance not located. ${ }^{13} \mathrm{C}$ NMR (benzene- $\left.d_{6}\right)$ : (Major isomer) $\square=-4.88\left(\mathrm{SiMe}{ }_{2}{ }^{\mathrm{t}} \mathrm{Bu}\right),-4.77\left(\mathrm{Si} M e_{2}{ }^{\mathrm{t}} \mathrm{Bu}\right), 18.20$ $\left.\left(\mathrm{SiMe}_{2} \mathrm{CMe}_{3}\right), 27.37\left(\mathrm{SiMe}_{2} \mathrm{CMe}\right)_{3}\right), 47.15,123.47,123.55$ (Cp), 123.57, 125.12, 128.97, 147.01, 148.33, 148.61 (Ind). (Minor Isomer) $\mathrm{Z}=-7.72\left(\mathrm{SiMe}{ }_{2}{ }^{\mathrm{t}} \mathrm{Bu}\right),-5.07\left(\mathrm{SiMe}{ }_{2}{ }^{\mathrm{B}} \mathrm{Bu}\right)$, $17.60\left(\mathrm{SiMe}_{2} \mathrm{CMe}_{3}\right), 27.21\left(\mathrm{SiMe}_{2} \mathrm{CMe}_{3}\right), 34.47,121.50123 .30$ (Cp), 123.87, 124.57, 125.30, 126.41, 136.42, 139.04 (Ind).

Preparation of $\mathrm{Li}\left[\mathrm{C}_{9} \mathrm{H}_{6}-\left(\mathrm{SiMe}_{2}{ }^{\mathrm{t}} \mathrm{Bu}\right)\right]$. This molecule was prepared in an identical manner to $\mathrm{Li}\left[\mathrm{C}_{9} \mathrm{H}_{6}-1-\left(\mathrm{SiMe}_{2} \mathrm{Ph}\right)\right]$ with $12.32 \mathrm{~g}(53.5 \mathrm{mmol})$ of $\mathrm{C}_{9} \mathrm{H}_{7}-\left(\mathrm{SiMe}_{2}{ }^{t} \mathrm{Bu}\right)$ and 36 $\mathrm{mL}$ (57.6 mmol) of 1.6 M ${ }^{\mathrm{n}} \mathrm{BuLi}$ yielding $8.84 \mathrm{~g}(72 \%)$ of an off-white solid. 
Preparation of $\mathrm{C}_{9} \mathbf{H}_{6}-1,3-\left(\mathrm{SiMe}_{2}{ }^{\mathrm{t}} \mathrm{Bu}\right)_{2}$. This molecule was prepared in a manner similar to $\mathrm{C}_{9} \mathrm{H}_{6}-1,3-\left(\mathrm{SiMe}_{2} \mathrm{Ph}\right)_{2}$ using $8.84 \mathrm{~g}(37.4 \mathrm{mmol})$ of $\mathrm{Li}\left[\mathrm{C}_{9} \mathrm{H}_{6}-\left(\mathrm{SiMe}_{2}{ }^{\mathrm{t}} \mathrm{Bu}\right)\right]$ and $5.64 \mathrm{~g}$ (37.4 mmol) of $\mathrm{SiMe}_{2}{ }^{\mathrm{t}} \mathrm{BuCl}$. Subsequent workup afforded $11.64 \mathrm{~g}(90 \%)$ of a waxy red solid identified as an equimolar mixture of two isomers of $\mathrm{C}_{9} \mathrm{H}_{6}-1,3-\left(\mathrm{SiMe}_{2}{ }^{\mathrm{t}} \mathrm{Bu}\right)_{2} \cdot{ }^{1} \mathrm{H}$ NMR (benzene- $d_{6}$ ): $\square=-0.47$ (s, $3 \mathrm{H}, \operatorname{Si} M e_{2}{ }^{\mathrm{t}} \mathrm{Bu}$ ), 0.00 (s, 3H, SiMe ${ }_{2}^{\mathrm{t}} \mathrm{Bu}$ ), 0.32 (s, $6 \mathrm{H}$, $\left.\mathrm{Si}_{2} e_{2}{ }^{\mathrm{B}} \mathrm{Bu}\right), 0.46$ (s, 3H, SiMe ${ }_{2}{ }^{\mathrm{t}} \mathrm{Bu}$ ), 0.54 (s, 3H, $\mathrm{Si} M e_{2}{ }^{\mathrm{t}} \mathrm{Bu}$ ), 0.81 (s, 9H, $\left.\mathrm{SiMe}_{2}{ }^{\mathrm{t}} \mathrm{Bu}\right), 1.01$ (s, 9H, $\left.\mathrm{SiMe}_{2}{ }^{t} \mathrm{Bu}\right), 1.07$ (s, 18H, $\left.\mathrm{SiMe}_{2}{ }^{t} \mathrm{Bu}\right), 3.52(\mathrm{~s}, 1 \mathrm{H}, \mathrm{Cp}), 7.01$ (s, 1H, Cp), 7.11 (m, 3H, Cp/Ind), 7.23 (m, 3H, Cp/Ind). 7.45 (d, 8 Hz, 1H, Ind), 7.71 (d, 8 Hz, 1H, Ind), 8.01 (m, 2H, Ind). One SiMe $e_{2}{ }^{\mathrm{B} u}$ resonance not located. ${ }^{13} \mathrm{C}$ NMR (benzene- $d_{6}$ ): $\square=-7.76$ $\left(\mathrm{Si} M e_{2}{ }^{\mathrm{t}} \mathrm{Bu}\right),-4.90\left(\mathrm{Si} M e_{2}{ }^{\mathrm{B}} \mathrm{Bu}\right),-4.71\left(\mathrm{Si} M e_{2}{ }^{\mathrm{t}} \mathrm{Bu}\right),-3.62\left(\mathrm{Si} M e_{2}{ }^{\mathrm{t}} \mathrm{Bu}\right),-3.25\left(\mathrm{Si} M e_{2}{ }^{\mathrm{t}} \mathrm{Bu}\right)$, $17.59\left(\mathrm{SiMe}_{2} \mathrm{CMe}_{3}\right), 18.20\left(\mathrm{SiMe}_{2} C \mathrm{Me}_{3}\right), 18.77\left(\mathrm{SiMe}_{2} \mathrm{CMe}_{3}\right), 27.21\left(\mathrm{SiMe}_{2} \mathrm{CMe}_{3}\right), 27.35$ $\left(\mathrm{SiMe}_{2} \mathrm{CMe}_{3}\right), 27.50\left(\mathrm{SiMe}_{2} \mathrm{CMe}_{3}\right), 47.14,100.64,118.12,123.12,123.47,123.55$ (Cp), $125.12,128.54,133.75,137.32,139.04,147.01,148.34,148.69$. Four Ind resonances not located.

Preparation of $\mathbf{K}\left[\mathrm{C}_{9} \mathbf{H}_{5}-\mathbf{1 , 3}-\left(\mathrm{SiMe}_{2}{ }^{\mathrm{t}} \mathrm{Bu}\right)_{2}\right]$. This molecule was prepared in a similar manner to $\mathrm{Li}\left[\mathrm{C}_{9} \mathrm{H}_{6}-1-\left(\mathrm{SiMe}_{2} \mathrm{Ph}\right)\right]$ with $5.37 \mathrm{~g}(15.6 \mathrm{mmol})$ of $\mathrm{C}_{9} \mathrm{H}_{6}-1,3-\left(\mathrm{SiMe}_{2}{ }^{\mathrm{B}} \mathrm{Bu}\right)_{2}$ and $3.11 \mathrm{~g}(15.6 \mathrm{mmol})$ of $\mathrm{K}\left[\mathrm{N}\left(\mathrm{SiMe}_{3}\right)_{2}\right]$ yielding $2.99 \mathrm{~g}(50 \%)$ of a light pink solid identified $\mathrm{K}_{2}\left[\mathrm{C}_{9} \mathrm{H}_{5}-1,3-\left(\mathrm{SiMe}_{2}{ }^{\mathrm{B}} \mathrm{Bu}\right)_{2}\right]$. Any traces of free amine was removed in vacuo by drying for several hours on a high vacuum line. 
Preparation of $\mathrm{C}_{9} \mathrm{H}_{6}-1-\left(\mathrm{SiMe}_{3}\right)-3-\left(\mathrm{CMe}_{3}\right)$. This molecule was prepared in an identical manner to $\mathrm{C}_{9} \mathrm{H}_{6}-1,3-\left(\mathrm{SiMe}_{2} \mathrm{Ph}\right)_{2}$ with $4.42 \mathrm{~g}(24.8 \mathrm{mmol})$ of $\mathrm{Li}\left[\mathrm{C}_{9} \mathrm{H}_{6}-\mathrm{CMe}_{3}\right]$ and $3.20 \mathrm{~mL}$ (25.0 mmol) of $\mathrm{Me}_{3} \mathrm{SiCl}$ yielding $6.01 \mathrm{~g}(99 \%)$ of a yellow oil identified as predominantly one isomer of $\mathrm{C}_{9} \mathrm{H}_{6}-1-\left(\mathrm{SiMe}_{3}\right)-3-\left(\mathrm{CMe}_{3}\right) .{ }^{1} \mathrm{H}$ NMR (benzene- $\left.d_{6}\right)$ : $\square=-0.22$ (s, 9H, SiMe $), 1.33$ (s, 9H, CMe 3 ), 3.65 (d, 4 Hz, 1H, Cp), 6.15 (d, 4 Hz, 1H, Cp), 7.10 (t, $4 \mathrm{~Hz}, 1 \mathrm{H}$, Ind), 7.17 (t, $4 \mathrm{~Hz}, 1 \mathrm{H}$, Ind), 7.34 (d, $4 \mathrm{~Hz}, 1 \mathrm{H}$, Ind), 7.62 (d, $4 \mathrm{~Hz}, 1 \mathrm{H}$, Ind). ${ }^{13} \mathrm{C}$ NMR (benzene- $\left.d_{6}\right):-\left[=-2.57(\mathrm{SiMe}), 29.95\left(\mathrm{CMe}_{3}\right), 33.31\left(\mathrm{CMe}_{3}\right), 43.91,122.53\right.$, 123.35 (Cp), 123.51, 124.64, 127.85, 143.21, 147.58, 151.09 (Ind).

Preparation of $\mathrm{Li}\left[\mathrm{C}_{9} \mathrm{H}_{5}-\mathbf{- 1}-\left(\mathrm{SiMe}_{3}\right)-3-\left(\mathrm{CMe}_{3}\right)\right]$. This molecule was prepared in an identical manner to $\mathrm{Li}\left[\mathrm{C}_{9} \mathrm{H}_{6}-1-\left(\mathrm{SiMe}_{2} \mathrm{Ph}\right)\right]$ with $6.01 \mathrm{~g}$ (24.6 mmol) of $\mathrm{C}_{9} \mathrm{H}_{6}-1-\left(\mathrm{SiMe}_{3}\right)-3-$ $\left(\mathrm{CMe}_{3}\right)$ and $17.0 \mathrm{~mL}(27.2 \mathrm{mmol})$ of $1.6 \mathrm{M}{ }^{\mathrm{n}} \mathrm{BuLi}$ yielding $5.60 \mathrm{~g}(91 \%)$ of an off-white solid. 
(a)

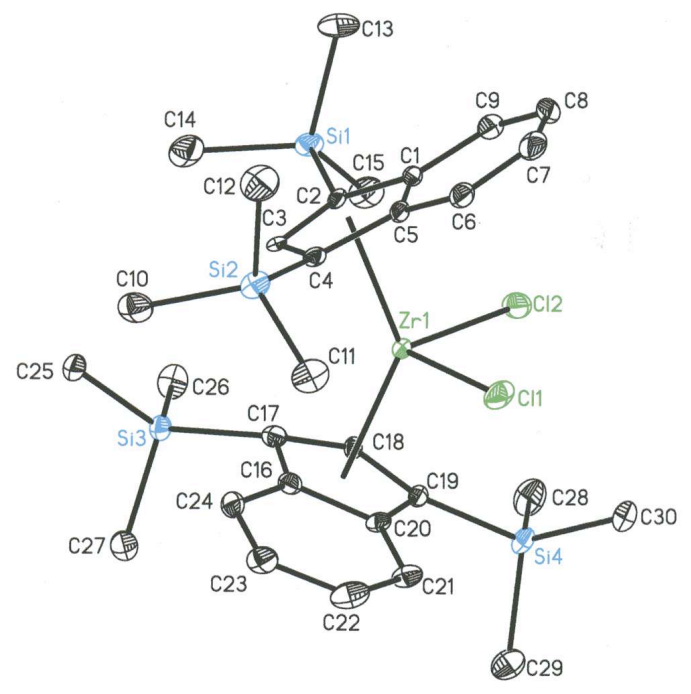

(b)

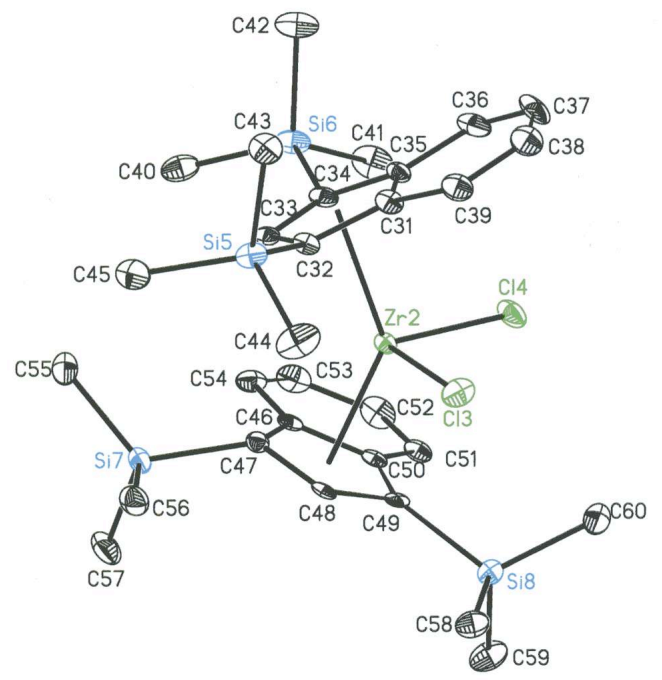

Figure S1. Atom labeling scheme for the solid state structure of $\mathbf{1 - C l}$. 
Table S1. Crystal data and structure refinement for $\mathbf{1 -} \mathbf{C l}_{\mathbf{2}}$.

Identification code

Empirical formula

Formula weight

Temperature

Wavelength

Crystal system

Space group

Unit cell dimensions

Volume

Z

Density (calculated)

Absorption coefficient

$\mathrm{F}(000)$

Crystal size

Theta range for data collection

Index ranges

Reflections collected

Independent reflections

Completeness to theta $=21.97^{\circ}$

Absorption correction

Max. and min. transmission

Refinement method

Data / restraints / parameters

Goodness-of-fit on $\mathrm{F}^{2}$

Final $\mathrm{R}$ indices $[\mathrm{I}>2 \operatorname{sigma}(\mathrm{I})]$

$\mathrm{R}$ indices (all data)

Largest diff. peak and hole
1-Cl

C30 H46 Cl2 Si4 Zr

681.15

173(2) K

$0.71073 \AA$

Triclinic

P-1

$\begin{array}{ll}\mathrm{a}=10.2583(10) \AA & \square=85.801(3)^{\circ} . \\ \mathrm{b}=17.7662(18) \AA & \square=85.434(3)^{\circ} . \\ \mathrm{c}=19.364(2) \AA & \square=87.321(3)^{\circ} .\end{array}$

3505.5(6) $\AA^{3}$

4

$1.291 \mathrm{Mg} / \mathrm{m}^{3}$

$0.621 \mathrm{~mm}^{-1}$

1424

$0.40 \times 0.30 \times 0.10 \mathrm{~mm}^{3}$

1.06 to $21.97^{\circ}$.

$-10<=\mathrm{h}<=9,-18<=\mathrm{k}<=18,-20<=\mathrm{l}<=13$

19189

$8350[\mathrm{R}($ int $)=0.0611]$

$97.4 \%$

\section{SADABS}

0.9405 and 0.6894

Full-matrix least-squares on $\mathrm{F}^{2}$

8350 / 0 / 667

1.056

$\mathrm{R} 1=0.0546, \mathrm{wR} 2=0.1082$

$\mathrm{R} 1=0.0827, \mathrm{wR} 2=0.1181$

0.635 and -0.418 e. $\AA^{-3}$ 
Table S2. Bond lengths $[\AA]$ and angles $\left[^{\circ}\right]$ for $\mathbf{1}-\mathbf{C l}_{\mathbf{2}}$.

\begin{tabular}{|c|c|c|c|}
\hline $\operatorname{Zr}(1)-\mathrm{Cl}(1)$ & $2.4032(13)$ & $C(5)-C(6)$ & $1.402(6)$ \\
\hline $\mathrm{Zr}(1)-\mathrm{Cl}(2)$ & $2.4239(13)$ & $C(6)-C(7)$ & $1.336(7)$ \\
\hline $\operatorname{Zr}(1)-C(18)$ & $2.484(4)$ & $\mathrm{C}(7)-\mathrm{C}(8)$ & $1.413(7)$ \\
\hline $\operatorname{Zr}(1)-C(3)$ & $2.496(4)$ & $\mathrm{C}(8)-\mathrm{C}(9)$ & $1.360(7)$ \\
\hline $\operatorname{Zr}(1)-C(19)$ & $2.507(4)$ & $C(16)-C(24)$ & $1.422(6)$ \\
\hline $\mathrm{Zr}(1)-\mathrm{C}(2)$ & $2.511(4)$ & $C(16)-C(20)$ & $1.452(6)$ \\
\hline $\operatorname{Zr}(1)-C(4)$ & $2.557(4)$ & $C(16)-C(17)$ & $1.439(6)$ \\
\hline $\operatorname{Zr}(1)-C(17)$ & $2.564(5)$ & $\mathrm{C}(17)-\mathrm{C}(18)$ & $1.421(6)$ \\
\hline $\operatorname{Zr}(1)-C(1)$ & $2.637(5)$ & $C(18)-C(19)$ & $1.409(6)$ \\
\hline $\operatorname{Zr}(1)-C(16)$ & $2.638(4)$ & $C(19)-C(20)$ & $1.433(6)$ \\
\hline $\operatorname{Zr}(1)-C(20)$ & $2.641(5)$ & $C(20)-C(21)$ & $1.401(6)$ \\
\hline $\operatorname{Zr}(1)-C(5)$ & $2.657(4)$ & $C(21)-C(22)$ & $1.358(6)$ \\
\hline $\operatorname{Si}(1)-C(14)$ & $1.847(5)$ & $C(22)-C(23)$ & $1.415(7)$ \\
\hline $\mathrm{Si}(1)-\mathrm{C}(13)$ & $1.856(5)$ & $C(23)-C(24)$ & $1.361(6)$ \\
\hline $\operatorname{Si}(1)-C(15)$ & $1.861(5)$ & $\operatorname{Zr}(2)-\mathrm{Cl}(4)$ & $2.4100(13)$ \\
\hline $\mathrm{Si}(1)-\mathrm{C}(2)$ & $1.870(4)$ & $\mathrm{Zr}(2)-\mathrm{Cl}(3)$ & $2.4148(13)$ \\
\hline $\operatorname{Si}(2)-C(11)$ & $1.857(5)$ & $\operatorname{Zr}(2)-C(48)$ & $2.485(4)$ \\
\hline $\mathrm{Si}(2)-\mathrm{C}(10)$ & $1.853(5)$ & $\operatorname{Zr}(2)-C(33)$ & 2.493(4) \\
\hline $\mathrm{Si}(2)-\mathrm{C}(12)$ & $1.867(6)$ & $\operatorname{Zr}(2)-C(32)$ & $2.500(5)$ \\
\hline $\mathrm{Si}(2)-\mathrm{C}(4)$ & $1.877(4)$ & $\operatorname{Zr}(2)-C(49)$ & $2.537(4)$ \\
\hline $\mathrm{Si}(3)-\mathrm{C}(27)$ & $1.860(5)$ & $\operatorname{Zr}(2)-C(47)$ & $2.544(4)$ \\
\hline $\mathrm{Si}(3)-\mathrm{C}(25)$ & $1.864(5)$ & $\mathrm{Zr}(2)-\mathrm{C}(34)$ & $2.570(5)$ \\
\hline $\mathrm{Si}(3)-\mathrm{C}(26)$ & $1.873(5)$ & $\operatorname{Zr}(2)-C(31)$ & $2.610(5)$ \\
\hline $\operatorname{Si}(3)-C(17)$ & $1.894(5)$ & $\operatorname{Zr}(2)-C(46)$ & $2.621(4)$ \\
\hline $\mathrm{Si}(4)-\mathrm{C}(30)$ & $1.863(5)$ & $\operatorname{Zr}(2)-C(50)$ & $2.635(4)$ \\
\hline $\mathrm{Si}(4)-\mathrm{C}(28)$ & $1.863(5)$ & $\operatorname{Zr}(2)-C(35)$ & $2.669(5)$ \\
\hline $\mathrm{Si}(4)-\mathrm{C}(29)$ & $1.875(5)$ & $\operatorname{Si}(5)-C(43)$ & $1.864(5)$ \\
\hline $\mathrm{Si}(4)-\mathrm{C}(19)$ & $1.887(4)$ & $\operatorname{Si}(5)-C(44)$ & $1.862(5)$ \\
\hline $\mathrm{C}(1)-\mathrm{C}(5)$ & $1.409(6)$ & $\operatorname{Si}(5)-C(45)$ & $1.859(5)$ \\
\hline $\mathrm{C}(1)-\mathrm{C}(9)$ & $1.418(6)$ & $\operatorname{Si}(5)-C(32)$ & $1.884(4)$ \\
\hline $\mathrm{C}(1)-\mathrm{C}(2)$ & $1.453(6)$ & $\operatorname{Si}(6)-C(41)$ & $1.856(5)$ \\
\hline $\mathrm{C}(2)-\mathrm{C}(3)$ & $1.433(6)$ & $\operatorname{Si}(6)-C(40)$ & $1.859(5)$ \\
\hline $\mathrm{C}(3)-\mathrm{C}(4)$ & $1.415(6)$ & $\operatorname{Si}(6)-C(34)$ & $1.881(5)$ \\
\hline $\mathrm{C}(4)-\mathrm{C}(5)$ & $1.449(6)$ & $\operatorname{Si}(6)-C(42)$ & $1.876(5)$ \\
\hline
\end{tabular}




\begin{tabular}{|c|c|c|c|}
\hline $\operatorname{Si}(7)-C(55)$ & $1.857(5)$ & $\mathrm{Cl}(2)-\operatorname{Zr}(1)-\mathrm{C}(19)$ & $82.65(10)$ \\
\hline $\operatorname{Si}(7)-C(56)$ & $1.858(5)$ & $\mathrm{C}(18)-\mathrm{Zr}(1)-\mathrm{C}(19)$ & $32.79(13)$ \\
\hline $\operatorname{Si}(7)-C(57)$ & $1.872(5)$ & $C(3)-\operatorname{Zr}(1)-C(19)$ & $135.38(14)$ \\
\hline $\operatorname{Si}(7)-C(47)$ & $1.882(5)$ & $\mathrm{Cl}(1)-\mathrm{Zr}(1)-\mathrm{C}(2)$ & $129.59(11)$ \\
\hline $\mathrm{Si}(8)-\mathrm{C}(58)$ & $1.855(5)$ & $\mathrm{Cl}(2)-\mathrm{Zr}(1)-\mathrm{C}(2)$ & $85.38(10)$ \\
\hline $\operatorname{Si}(8)-C(59)$ & $1.867(5)$ & $C(18)-\operatorname{Zr}(1)-C(2)$ & $106.86(14)$ \\
\hline $\operatorname{Si}(8)-C(60)$ & $1.865(5)$ & $C(3)-\operatorname{Zr}(1)-C(2)$ & $33.26(13)$ \\
\hline $\operatorname{Si}(8)-C(49)$ & $1.876(5)$ & $C(19)-\operatorname{Zr}(1)-C(2)$ & $138.88(14)$ \\
\hline$C(31)-C(39)$ & $1.418(7)$ & $\mathrm{Cl}(1)-\mathrm{Zr}(1)-\mathrm{C}(4)$ & $89.00(10)$ \\
\hline$C(31)-C(35)$ & $1.413(6)$ & $\mathrm{Cl}(2)-\mathrm{Zr}(1)-\mathrm{C}(4)$ & $130.70(10)$ \\
\hline $\mathrm{C}(31)-\mathrm{C}(32)$ & $1.446(6)$ & $C(18)-\operatorname{Zr}(1)-C(4)$ & $131.90(14)$ \\
\hline$C(32)-C(33)$ & $1.412(6)$ & $C(3)-\operatorname{Zr}(1)-C(4)$ & $32.49(14)$ \\
\hline$C(33)-C(34)$ & $1.425(6)$ & $C(19)-\operatorname{Zr}(1)-C(4)$ & $146.39(14)$ \\
\hline$C(34)-C(35)$ & $1.446(6)$ & $C(2)-\operatorname{Zr}(1)-C(4)$ & $55.37(14)$ \\
\hline $\mathrm{C}(35)-\mathrm{C}(36)$ & $1.434(6)$ & $\mathrm{Cl}(1)-\mathrm{Zr}(1)-\mathrm{C}(17)$ & $127.92(11)$ \\
\hline$C(36)-C(37)$ & $1.361(7)$ & $\mathrm{Cl}(2)-\mathrm{Zr}(1)-\mathrm{C}(17)$ & $113.54(10)$ \\
\hline $\mathrm{C}(37)-\mathrm{C}(38)$ & $1.394(7)$ & $\mathrm{C}(18)-\mathrm{Zr}(1)-\mathrm{C}(17)$ & $32.65(13)$ \\
\hline C(38)-C(39) & $1.355(7)$ & $C(3)-\operatorname{Zr}(1)-C(17)$ & $80.39(14)$ \\
\hline$C(46)-C(54)$ & $1.402(6)$ & $C(19)-\operatorname{Zr}(1)-C(17)$ & $55.01(14)$ \\
\hline$C(46)-C(50)$ & $1.424(6)$ & $C(2)-\operatorname{Zr}(1)-C(17)$ & $95.47(14)$ \\
\hline $\mathrm{C}(46)-\mathrm{C}(47)$ & $1.452(6)$ & $C(4)-\operatorname{Zr}(1)-C(17)$ & $100.04(14)$ \\
\hline $\mathrm{C}(47)-\mathrm{C}(48)$ & $1.409(6)$ & $\mathrm{Cl}(1)-\mathrm{Zr}(1)-\mathrm{C}(1)$ & $98.47(10)$ \\
\hline C(48)-C(49) & $1.414(6)$ & $\mathrm{Cl}(2)-\mathrm{Zr}(1)-\mathrm{C}(1)$ & $77.72(10)$ \\
\hline$C(49)-C(50)$ & $1.442(6)$ & $C(18)-\operatorname{Zr}(1)-C(1)$ & $135.84(14)$ \\
\hline$C(50)-C(51)$ & $1.410(6)$ & $C(3)-\operatorname{Zr}(1)-C(1)$ & $52.67(14)$ \\
\hline$C(51)-C(52)$ & $1.355(7)$ & $C(19)-\operatorname{Zr}(1)-C(1)$ & $159.07(14)$ \\
\hline$C(52)-C(53)$ & $1.410(7)$ & $C(2)-\operatorname{Zr}(1)-C(1)$ & $32.67(14)$ \\
\hline \multirow[t]{2}{*}{$C(53)-C(54)$} & $1.362(7)$ & $C(4)-\operatorname{Zr}(1)-C(1)$ & $53.04(13)$ \\
\hline & & $C(17)-\operatorname{Zr}(1)-C(1)$ & $127.79(14)$ \\
\hline $\mathrm{Cl}(1)-\mathrm{Zr}(1)-\mathrm{Cl}(2)$ & $96.86(5)$ & $\mathrm{Cl}(1)-\mathrm{Zr}(1)-\mathrm{C}(16)$ & $96.92(10)$ \\
\hline $\mathrm{Cl}(1)-\mathrm{Zr}(1)-\mathrm{C}(18)$ & $123.48(11)$ & $\mathrm{Cl}(2)-\mathrm{Zr}(1)-\mathrm{C}(16)$ & $134.21(10)$ \\
\hline $\mathrm{Cl}(2)-\operatorname{Zr}(1)-\mathrm{C}(18)$ & $83.71(10)$ & $\mathrm{C}(18)-\mathrm{Zr}(1)-\mathrm{C}(16)$ & $52.63(14)$ \\
\hline $\mathrm{Cl}(1)-\mathrm{Zr}(1)-\mathrm{C}(3)$ & $121.46(10)$ & $C(3)-\operatorname{Zr}(1)-C(16)$ & $89.88(14)$ \\
\hline $\mathrm{Cl}(2)-\mathrm{Zr}(1)-\mathrm{C}(3)$ & $118.56(10)$ & $\mathrm{C}(19)-\operatorname{Zr}(1)-\mathrm{C}(16)$ & $53.69(14)$ \\
\hline$C(18)-\operatorname{Zr}(1)-C(3)$ & $106.43(14)$ & $C(2)-\operatorname{Zr}(1)-C(16)$ & $117.11(14)$ \\
\hline $\mathrm{Cl}(1)-\operatorname{Zr}(1)-\mathrm{C}(19)$ & $90.93(10)$ & $C(4)-\operatorname{Zr}(1)-C(16)$ & $92.97(14)$ \\
\hline
\end{tabular}




\begin{tabular}{|c|c|}
\hline $\mathrm{C}(17)-\mathrm{Zr}(1)-\mathrm{C}(16)$ & $32.08(13)$ \\
\hline $\mathrm{C}(1)-\mathrm{Zr}(1)-\mathrm{C}(16)$ & $142.08(14)$ \\
\hline $\mathrm{Cl}(1)-\operatorname{Zr}(1)-\mathrm{C}(20)$ & $76.30(10)$ \\
\hline $\mathrm{Cl}(2)-\operatorname{Zr}(1)-\mathrm{C}(20)$ & $112.60(10)$ \\
\hline$C(18)-\operatorname{Zr}(1)-C(20)$ & $52.72(14)$ \\
\hline$C(3)-\operatorname{Zr}(1)-C(20)$ & $121.42(14)$ \\
\hline$C(19)-\operatorname{Zr}(1)-C(20)$ & $32.20(13)$ \\
\hline$C(2)-\operatorname{Zr}(1)-C(20)$ & $148.03(14)$ \\
\hline $\mathrm{C}(4)-\mathrm{Zr}(1)-\mathrm{C}(20)$ & $116.32(14)$ \\
\hline $\mathrm{C}(17)-\operatorname{Zr}(1)-\mathrm{C}(20)$ & $53.61(14)$ \\
\hline $\mathrm{C}(1)-\mathrm{Zr}(1)-\mathrm{C}(20)$ & $168.72(13)$ \\
\hline$C(16)-\operatorname{Zr}(1)-C(20)$ & $31.93(14)$ \\
\hline $\mathrm{Cl}(1)-\mathrm{Zr}(1)-\mathrm{C}(5)$ & $76.93(10)$ \\
\hline $\mathrm{Cl}(2)-\mathrm{Zr}(1)-\mathrm{C}(5)$ & $102.00(10)$ \\
\hline$C(18)-\operatorname{Zr}(1)-C(5)$ & $158.40(14)$ \\
\hline$C(3)-\operatorname{Zr}(1)-C(5)$ & $52.45(14)$ \\
\hline$C(19)-\operatorname{Zr}(1)-C(5)$ & $167.38(14)$ \\
\hline$C(2)-\operatorname{Zr}(1)-C(5)$ & $53.67(14)$ \\
\hline$C(4)-\operatorname{Zr}(1)-C(5)$ & $32.18(13)$ \\
\hline$C(17)-\operatorname{Zr}(1)-C(5)$ & $130.85(14)$ \\
\hline$C(1)-\operatorname{Zr}(1)-C(5)$ & $30.86(13)$ \\
\hline$C(16)-\operatorname{Zr}(1)-C(5)$ & $123.63(14)$ \\
\hline$C(20)-\operatorname{Zr}(1)-C(5)$ & $138.17(14)$ \\
\hline $\mathrm{C}(14)-\mathrm{Si}(1)-\mathrm{C}(13)$ & $106.9(3)$ \\
\hline C(14)-Si(1)-C(15) & $111.1(2)$ \\
\hline$C(13)-\operatorname{Si}(1)-C(15)$ & $108.1(2)$ \\
\hline $\mathrm{C}(14)-\mathrm{Si}(1)-\mathrm{C}(2)$ & $107.0(2)$ \\
\hline$C(13)-\operatorname{Si}(1)-C(2)$ & $108.6(2)$ \\
\hline $\mathrm{C}(15)-\mathrm{Si}(1)-\mathrm{C}(2)$ & $114.8(2)$ \\
\hline$C(11)-\operatorname{Si}(2)-C(10)$ & $113.4(2)$ \\
\hline $\mathrm{C}(11)-\mathrm{Si}(2)-\mathrm{C}(12)$ & 107.7(3) \\
\hline $\mathrm{C}(10)-\mathrm{Si}(2)-\mathrm{C}(12)$ & 107.2(3) \\
\hline C(11)-Si(2)-C(4) & $112.6(2)$ \\
\hline $\mathrm{C}(10)-\mathrm{Si}(2)-\mathrm{C}(4)$ & $109.5(2)$ \\
\hline$C(12)-\operatorname{Si}(2)-C(4)$ & $106.0(2)$ \\
\hline $\mathrm{C}(27)-\mathrm{Si}(3)-\mathrm{C}(25)$ & $108.0(2)$ \\
\hline
\end{tabular}

\begin{tabular}{|c|c|}
\hline $\mathrm{C}(27)-\mathrm{Si}(3)-\mathrm{C}(26)$ & $108.3(2)$ \\
\hline C(25)-Si(3)-C(26) & $112.0(2)$ \\
\hline $\mathrm{C}(27)-\mathrm{Si}(3)-\mathrm{C}(17)$ & $103.3(2)$ \\
\hline $\mathrm{C}(25)-\mathrm{Si}(3)-\mathrm{C}(17)$ & $115.0(2)$ \\
\hline $\mathrm{C}(26)-\mathrm{Si}(3)-\mathrm{C}(17)$ & $109.6(2)$ \\
\hline $\mathrm{C}(30)-\mathrm{Si}(4)-\mathrm{C}(28)$ & $111.9(2)$ \\
\hline $\mathrm{C}(30)-\mathrm{Si}(4)-\mathrm{C}(29)$ & $108.0(2)$ \\
\hline$C(28)-\operatorname{Si}(4)-C(29)$ & $109.2(2)$ \\
\hline $\mathrm{C}(30)-\mathrm{Si}(4)-\mathrm{C}(19)$ & $114.3(2)$ \\
\hline $\mathrm{C}(28)-\mathrm{Si}(4)-\mathrm{C}(19)$ & $107.5(2)$ \\
\hline $\mathrm{C}(29)-\mathrm{Si}(4)-\mathrm{C}(19)$ & $105.6(2)$ \\
\hline C(5)-C(1)-C(9) & 119.1(4) \\
\hline$C(5)-C(1)-C(2)$ & $109.5(4)$ \\
\hline $\mathrm{C}(9)-\mathrm{C}(1)-\mathrm{C}(2)$ & $131.4(4)$ \\
\hline$C(5)-C(1)-Z r(1)$ & $75.3(3)$ \\
\hline $\mathrm{C}(9)-\mathrm{C}(1)-\mathrm{Zr}(1)$ & $122.5(3)$ \\
\hline $\mathrm{C}(2)-\mathrm{C}(1)-\mathrm{Zr}(1)$ & $68.9(2)$ \\
\hline $\mathrm{C}(3)-\mathrm{C}(2)-\mathrm{C}(1)$ & $104.4(4)$ \\
\hline $\mathrm{C}(3)-\mathrm{C}(2)-\mathrm{Si}(1)$ & $126.8(3)$ \\
\hline $\mathrm{C}(1)-\mathrm{C}(2)-\mathrm{Si}(1)$ & $125.3(3)$ \\
\hline$C(3)-C(2)-\operatorname{Zr}(1)$ & $72.8(2)$ \\
\hline $\mathrm{C}(1)-\mathrm{C}(2)-\mathrm{Zr}(1)$ & $78.5(3)$ \\
\hline $\operatorname{Si}(1)-C(2)-\operatorname{Zr}(1)$ & $130.0(2)$ \\
\hline$C(4)-C(3)-C(2)$ & $111.6(4)$ \\
\hline$C(4)-C(3)-Z r(1)$ & $76.1(3)$ \\
\hline$C(2)-C(3)-\operatorname{Zr}(1)$ & $73.9(2)$ \\
\hline$C(3)-C(4)-C(5)$ & $105.7(4)$ \\
\hline $\mathrm{C}(3)-\mathrm{C}(4)-\mathrm{Si}(2)$ & $127.6(3)$ \\
\hline $\mathrm{C}(5)-\mathrm{C}(4)-\mathrm{Si}(2)$ & $123.1(3)$ \\
\hline$C(3)-C(4)-Z r(1)$ & $71.4(2)$ \\
\hline$C(5)-C(4)-\operatorname{Zr}(1)$ & $77.7(3)$ \\
\hline $\operatorname{Si}(2)-C(4)-Z r(1)$ & $133.2(2)$ \\
\hline$C(6)-C(5)-C(1)$ & $120.4(4)$ \\
\hline$C(6)-C(5)-C(4)$ & $130.9(4)$ \\
\hline$C(1)-C(5)-C(4)$ & $108.6(4)$ \\
\hline$C(6)-C(5)-Z r(1)$ & $125.4(3)$ \\
\hline
\end{tabular}




\begin{tabular}{|c|c|}
\hline$C(1)-C(5)-Z r(1)$ & $73.8(3)$ \\
\hline$C(4)-C(5)-Z r(1)$ & $70.1(2)$ \\
\hline$C(7)-C(6)-C(5)$ & $119.2(5)$ \\
\hline$C(6)-C(7)-C(8)$ & $121.6(5)$ \\
\hline $\mathrm{C}(9)-\mathrm{C}(8)-\mathrm{C}(7)$ & $120.8(5)$ \\
\hline $\mathrm{C}(8)-\mathrm{C}(9)-\mathrm{C}(1)$ & $118.8(5)$ \\
\hline$C(24)-C(16)-C(20)$ & 119.1(4) \\
\hline$C(24)-C(16)-C(17)$ & $131.8(4)$ \\
\hline$C(20)-C(16)-C(17)$ & $108.6(4)$ \\
\hline$C(24)-C(16)-\operatorname{Zr}(1)$ & $127.0(3)$ \\
\hline$C(20)-C(16)-Z r(1)$ & $74.1(3)$ \\
\hline$C(17)-C(16)-\operatorname{Zr}(1)$ & $71.1(3)$ \\
\hline $\mathrm{C}(18)-\mathrm{C}(17)-\mathrm{C}(16)$ & $105.4(4)$ \\
\hline C(18)-C(17)-Si(3) & $121.5(3)$ \\
\hline $\mathrm{C}(16)-\mathrm{C}(17)-\mathrm{Si}(3)$ & $123.4(3)$ \\
\hline $\mathrm{C}(18)-\mathrm{C}(17)-\mathrm{Zr}(1)$ & $70.6(2)$ \\
\hline$C(16)-C(17)-Z r(1)$ & $76.8(3)$ \\
\hline $\operatorname{Si}(3)-C(17)-\operatorname{Zr}(1)$ & $145.0(2)$ \\
\hline C(19)-C(18)-C(17) & $111.7(4)$ \\
\hline$C(19)-C(18)-\operatorname{Zr}(1)$ & $74.5(2)$ \\
\hline $\mathrm{C}(17)-\mathrm{C}(18)-\mathrm{Zr}(1)$ & $76.8(3)$ \\
\hline $\mathrm{C}(18)-\mathrm{C}(19)-\mathrm{C}(20)$ & $106.6(4)$ \\
\hline C(18)-C(19)-Si(4) & $124.3(3)$ \\
\hline C(20)-C(19)-Si(4) & $127.2(3)$ \\
\hline$C(18)-C(19)-\operatorname{Zr}(1)$ & $72.7(2)$ \\
\hline$C(20)-C(19)-Z r(1)$ & $79.0(3)$ \\
\hline $\operatorname{Si}(4)-C(19)-\operatorname{Zr}(1)$ & $126.0(2)$ \\
\hline $\mathrm{C}(21)-\mathrm{C}(20)-\mathrm{C}(19)$ & $133.0(4)$ \\
\hline $\mathrm{C}(21)-\mathrm{C}(20)-\mathrm{C}(16)$ & $119.5(4)$ \\
\hline$C(19)-C(20)-C(16)$ & $107.5(4)$ \\
\hline$C(21)-C(20)-Z r(1)$ & $124.4(3)$ \\
\hline$C(19)-C(20)-\operatorname{Zr}(1)$ & $68.8(2)$ \\
\hline$C(16)-C(20)-Z r(1)$ & $73.9(3)$ \\
\hline $\mathrm{C}(22)-\mathrm{C}(21)-\mathrm{C}(20)$ & $119.1(5)$ \\
\hline$C(21)-C(22)-C(23)$ & $122.3(5)$ \\
\hline $\mathrm{C}(24)-\mathrm{C}(23)-\mathrm{C}(22)$ & $120.7(4)$ \\
\hline
\end{tabular}

\begin{tabular}{|c|c|}
\hline$C(23)-C(24)-C(16)$ & 119.3(4) \\
\hline $\mathrm{Cl}(4)-\mathrm{Zr}(2)-\mathrm{Cl}(3)$ & $97.38(5)$ \\
\hline $\mathrm{Cl}(4)-\mathrm{Zr}(2)-\mathrm{C}(48)$ & $121.03(11)$ \\
\hline $\mathrm{Cl}(3)-\mathrm{Zr}(2)-\mathrm{C}(48)$ & $80.68(11)$ \\
\hline $\mathrm{Cl}(4)-\operatorname{Zr}(2)-\mathrm{C}(33)$ & $121.96(10)$ \\
\hline $\mathrm{Cl}(3)-\mathrm{Zr}(2)-\mathrm{C}(33)$ & $118.27(11)$ \\
\hline $\mathrm{C}(48)-\operatorname{Zr}(2)-\mathrm{C}(33)$ & $109.43(14)$ \\
\hline $\mathrm{Cl}(4)-\mathrm{Zr}(2)-\mathrm{C}(32)$ & $128.26(10)$ \\
\hline $\mathrm{Cl}(3)-\mathrm{Zr}(2)-\mathrm{C}(32)$ & $85.69(10)$ \\
\hline $\mathrm{C}(48)-\operatorname{Zr}(2)-\mathrm{C}(32)$ & $110.48(14)$ \\
\hline $\mathrm{C}(33)-\operatorname{Zr}(2)-\mathrm{C}(32)$ & $32.85(14)$ \\
\hline $\mathrm{Cl}(4)-\operatorname{Zr}(2)-\mathrm{C}(49)$ & $88.36(11)$ \\
\hline $\mathrm{Cl}(3)-\mathrm{Zr}(2)-\mathrm{C}(49)$ & $83.25(10)$ \\
\hline $\mathrm{C}(48)-\mathrm{Zr}(2)-\mathrm{C}(49)$ & $32.67(14)$ \\
\hline $\mathrm{C}(33)-\mathrm{Zr}(2)-\mathrm{C}(49)$ & $136.56(14)$ \\
\hline$C(32)-\operatorname{Zr}(2)-C(49)$ & $142.87(14)$ \\
\hline $\mathrm{Cl}(4)-\operatorname{Zr}(2)-\mathrm{C}(47)$ & $130.29(11)$ \\
\hline $\mathrm{Cl}(3)-\mathrm{Zr}(2)-\mathrm{C}(47)$ & $108.71(10)$ \\
\hline $\mathrm{C}(48)-\operatorname{Zr}(2)-\mathrm{C}(47)$ & $32.52(14)$ \\
\hline $\mathrm{C}(33)-\mathrm{Zr}(2)-\mathrm{C}(47)$ & $81.61(15)$ \\
\hline$C(32)-Z r(2)-C(47)$ & $95.98(15)$ \\
\hline $\mathrm{C}(49)-\operatorname{Zr}(2)-\mathrm{C}(47)$ & $55.14(14)$ \\
\hline $\mathrm{Cl}(4)-\operatorname{Zr}(2)-\mathrm{C}(34)$ & $89.47(11)$ \\
\hline $\mathrm{Cl}(3)-\operatorname{Zr}(2)-\mathrm{C}(34)$ & $133.03(11)$ \\
\hline$C(48)-\operatorname{Zr}(2)-C(34)$ & $133.62(15)$ \\
\hline $\mathrm{C}(33)-\operatorname{Zr}(2)-\mathrm{C}(34)$ & $32.64(14)$ \\
\hline $\mathrm{C}(32)-\mathrm{Zr}(2)-\mathrm{C}(34)$ & $55.39(14)$ \\
\hline $\mathrm{C}(49)-\operatorname{Zr}(2)-\mathrm{C}(34)$ & $143.59(14)$ \\
\hline $\mathrm{C}(47)-\mathrm{Zr}(2)-\mathrm{C}(34)$ & $101.25(15)$ \\
\hline $\mathrm{Cl}(4)-\operatorname{Zr}(2)-\mathrm{C}(31)$ & $96.78(11)$ \\
\hline $\mathrm{Cl}(3)-\mathrm{Zr}(2)-\mathrm{C}(31)$ & $79.49(11)$ \\
\hline $\mathrm{C}(48)-\mathrm{Zr}(2)-\mathrm{C}(31)$ & $139.12(14)$ \\
\hline $\mathrm{C}(33)-\operatorname{Zr}(2)-\mathrm{C}(31)$ & $52.72(15)$ \\
\hline$C(32)-\operatorname{Zr}(2)-C(31)$ & $32.78(14)$ \\
\hline $\mathrm{C}(49)-\operatorname{Zr}(2)-\mathrm{C}(31)$ & $162.47(14)$ \\
\hline $\mathrm{C}(47)-\operatorname{Zr}(2)-\mathrm{C}(31)$ & $128.58(15)$ \\
\hline
\end{tabular}




\begin{tabular}{|c|c|}
\hline$C(34)-\operatorname{Zr}(2)-C(31)$ & $53.54(14)$ \\
\hline $\mathrm{Cl}(4)-\mathrm{Zr}(2)-\mathrm{C}(46)$ & $99.88(10)$ \\
\hline $\mathrm{Cl}(3)-\mathrm{Zr}(2)-\mathrm{C}(46)$ & $132.50(10)$ \\
\hline$C(48)-\operatorname{Zr}(2)-C(46)$ & $52.57(14)$ \\
\hline$C(33)-\operatorname{Zr}(2)-C(46)$ & $88.45(14)$ \\
\hline$C(32)-\operatorname{Zr}(2)-C(46)$ & $115.61(14)$ \\
\hline$C(49)-\operatorname{Zr}(2)-C(46)$ & $53.60(14)$ \\
\hline$C(47)-\operatorname{Zr}(2)-C(46)$ & $32.62(13)$ \\
\hline$C(34)-\operatorname{Zr}(2)-C(46)$ & $91.13(14)$ \\
\hline$C(31)-\operatorname{Zr}(2)-C(46)$ & $140.66(14)$ \\
\hline $\mathrm{Cl}(4)-\mathrm{Zr}(2)-\mathrm{C}(50)$ & $77.23(10)$ \\
\hline $\mathrm{Cl}(3)-\mathrm{Zr}(2)-\mathrm{C}(50)$ & $114.55(10)$ \\
\hline$C(48)-\operatorname{Zr}(2)-C(50)$ & $52.46(14)$ \\
\hline$C(33)-\operatorname{Zr}(2)-C(50)$ & $118.95(14)$ \\
\hline$C(32)-\operatorname{Zr}(2)-C(50)$ & $146.81(14)$ \\
\hline$C(49)-\operatorname{Zr}(2)-C(50)$ & $32.31(13)$ \\
\hline$C(47)-\operatorname{Zr}(2)-C(50)$ & $53.67(14)$ \\
\hline$C(34)-\operatorname{Zr}(2)-C(50)$ & $112.29(14)$ \\
\hline$C(31)-\operatorname{Zr}(2)-C(50)$ & $165.13(14)$ \\
\hline$C(46)-\operatorname{Zr}(2)-C(50)$ & $31.44(14)$ \\
\hline $\mathrm{Cl}(4)-\mathrm{Zr}(2)-\mathrm{C}(35)$ & $76.49(10)$ \\
\hline $\mathrm{Cl}(3)-\mathrm{Zr}(2)-\mathrm{C}(35)$ & $105.14(11)$ \\
\hline $\mathrm{C}(48)-\mathrm{Zr}(2)-\mathrm{C}(35)$ & $161.20(14)$ \\
\hline$C(33)-\operatorname{Zr}(2)-C(35)$ & $51.93(14)$ \\
\hline$C(32)-\operatorname{Zr}(2)-C(35)$ & $53.30(14)$ \\
\hline$C(49)-\operatorname{Zr}(2)-C(35)$ & $163.40(14)$ \\
\hline$C(47)-\operatorname{Zr}(2)-C(35)$ & $131.68(14)$ \\
\hline$C(34)-\operatorname{Zr}(2)-C(35)$ & $31.97(14)$ \\
\hline$C(31)-\operatorname{Zr}(2)-C(35)$ & $31.03(14)$ \\
\hline$C(46)-\operatorname{Zr}(2)-C(35)$ & $121.82(14)$ \\
\hline$C(50)-\operatorname{Zr}(2)-C(35)$ & $134.51(13)$ \\
\hline $\mathrm{C}(43)-\mathrm{Si}(5)-\mathrm{C}(44)$ & $109.8(3)$ \\
\hline C(43)-Si(5)-C(45) & $107.8(2)$ \\
\hline C(44)-Si(5)-C(45) & 109.6(3) \\
\hline $\mathrm{C}(43)-\mathrm{Si}(5)-\mathrm{C}(32)$ & $104.4(2)$ \\
\hline $\mathrm{C}(44)-\mathrm{Si}(5)-\mathrm{C}(32)$ & $114.2(2)$ \\
\hline
\end{tabular}

\begin{tabular}{|c|c|}
\hline $\mathrm{C}(45)-\mathrm{Si}(5)-\mathrm{C}(32)$ & $110.8(2)$ \\
\hline$C(41)-\operatorname{Si}(6)-C(40)$ & $112.2(2)$ \\
\hline$C(41)-\operatorname{Si}(6)-C(34)$ & $111.7(2)$ \\
\hline$C(40)-S i(6)-C(34)$ & $112.6(2)$ \\
\hline $\mathrm{C}(41)-\mathrm{Si}(6)-\mathrm{C}(42)$ & $108.2(3)$ \\
\hline $\mathrm{C}(40)-\mathrm{Si}(6)-\mathrm{C}(42)$ & $107.9(3)$ \\
\hline$C(34)-\operatorname{Si}(6)-C(42)$ & $103.8(2)$ \\
\hline$C(55)-\operatorname{Si}(7)-C(56)$ & $111.8(2)$ \\
\hline $\mathrm{C}(55)-\mathrm{Si}(7)-\mathrm{C}(57)$ & $107.2(2)$ \\
\hline$C(56)-\operatorname{Si}(7)-C(57)$ & $109.6(2)$ \\
\hline $\mathrm{C}(55)-\mathrm{Si}(7)-\mathrm{C}(47)$ & $116.6(2)$ \\
\hline $\mathrm{C}(56)-\mathrm{Si}(7)-\mathrm{C}(47)$ & $107.5(2)$ \\
\hline $\mathrm{C}(57)-\mathrm{Si}(7)-\mathrm{C}(47)$ & $103.7(2)$ \\
\hline $\mathrm{C}(58)-\mathrm{Si}(8)-\mathrm{C}(59)$ & $109.4(2)$ \\
\hline $\mathrm{C}(58)-\mathrm{Si}(8)-\mathrm{C}(60)$ & $110.0(2)$ \\
\hline C(59)-Si(8)-C(60) & $108.5(3)$ \\
\hline C(58)-Si(8)-C(49) & $108.1(2)$ \\
\hline $\mathrm{C}(59)-\mathrm{Si}(8)-\mathrm{C}(49)$ & $105.9(2)$ \\
\hline $\mathrm{C}(60)-\mathrm{Si}(8)-\mathrm{C}(49)$ & $114.9(2)$ \\
\hline$C(39)-C(31)-C(35)$ & $118.9(4)$ \\
\hline $\mathrm{C}(39)-\mathrm{C}(31)-\mathrm{C}(32)$ & $132.4(4)$ \\
\hline $\mathrm{C}(35)-\mathrm{C}(31)-\mathrm{C}(32)$ & $108.7(4)$ \\
\hline $\mathrm{C}(39)-\mathrm{C}(31)-\mathrm{Zr}(2)$ & $119.6(3)$ \\
\hline $\mathrm{C}(35)-\mathrm{C}(31)-\mathrm{Zr}(2)$ & $76.8(3)$ \\
\hline $\mathrm{C}(32)-\mathrm{C}(31)-\mathrm{Zr}(2)$ & $69.4(3)$ \\
\hline $\mathrm{C}(33)-\mathrm{C}(32)-\mathrm{C}(31)$ & $105.1(4)$ \\
\hline$C(33)-C(32)-\operatorname{Si}(5)$ & $128.0(3)$ \\
\hline$C(31)-C(32)-\operatorname{Si}(5)$ & $121.5(3)$ \\
\hline$C(33)-C(32)-\operatorname{Zr}(2)$ & $73.3(3)$ \\
\hline$C(31)-C(32)-\operatorname{Zr}(2)$ & $77.8(3)$ \\
\hline $\operatorname{Si}(5)-C(32)-\operatorname{Zr}(2)$ & $134.6(2)$ \\
\hline $\mathrm{C}(32)-\mathrm{C}(33)-\mathrm{C}(34)$ & $112.4(4)$ \\
\hline $\mathrm{C}(32)-\mathrm{C}(33)-\operatorname{Zr}(2)$ & $73.8(3)$ \\
\hline $\mathrm{C}(34)-\mathrm{C}(33)-\operatorname{Zr}(2)$ & $76.6(3)$ \\
\hline $\mathrm{C}(33)-\mathrm{C}(34)-\mathrm{C}(35)$ & $104.2(4)$ \\
\hline$C(33)-C(34)-\operatorname{Si}(6)$ & $127.5(4)$ \\
\hline
\end{tabular}




\begin{tabular}{|c|c|c|c|}
\hline$C(35)-C(34)-\operatorname{Si}(6)$ & $122.6(3)$ & $\mathrm{C}(48)-\mathrm{C}(47)-\operatorname{Zr}(2)$ & $71.4(2)$ \\
\hline$C(33)-C(34)-\operatorname{Zr}(2)$ & $70.7(2)$ & $\mathrm{C}(46)-\mathrm{C}(47)-\mathrm{Zr}(2)$ & $76.6(2)$ \\
\hline$C(35)-C(34)-\operatorname{Zr}(2)$ & $77.8(3)$ & $\operatorname{Si}(7)-C(47)-Z r(2)$ & $143.2(2)$ \\
\hline $\operatorname{Si}(6)-C(34)-\operatorname{Zr}(2)$ & $136.9(2)$ & $\mathrm{C}(49)-\mathrm{C}(48)-\mathrm{C}(47)$ & $112.9(4)$ \\
\hline$C(31)-C(35)-C(36)$ & $119.8(4)$ & $\mathrm{C}(49)-\mathrm{C}(48)-\operatorname{Zr}(2)$ & $75.7(2)$ \\
\hline $\mathrm{C}(31)-\mathrm{C}(35)-\mathrm{C}(34)$ & $109.4(4)$ & $\mathrm{C}(47)-\mathrm{C}(48)-\operatorname{Zr}(2)$ & $76.1(3)$ \\
\hline$C(36)-C(35)-C(34)$ & $130.8(4)$ & $C(48)-C(49)-C(50)$ & $105.1(4)$ \\
\hline$C(31)-C(35)-\operatorname{Zr}(2)$ & $72.2(3)$ & $\mathrm{C}(48)-\mathrm{C}(49)-\mathrm{Si}(8)$ & $125.0(3)$ \\
\hline$C(36)-C(35)-\operatorname{Zr}(2)$ & $124.3(3)$ & $C(50)-C(49)-\operatorname{Si}(8)$ & $128.6(3)$ \\
\hline$C(34)-C(35)-\operatorname{Zr}(2)$ & $70.2(3)$ & $C(48)-C(49)-\operatorname{Zr}(2)$ & $71.6(2)$ \\
\hline$C(37)-C(36)-C(35)$ & $118.3(5)$ & $C(50)-C(49)-Z r(2)$ & $77.6(2)$ \\
\hline $\mathrm{C}(36)-\mathrm{C}(37)-\mathrm{C}(38)$ & $121.8(5)$ & $\operatorname{Si}(8)-C(49)-\operatorname{Zr}(2)$ & $125.8(2)$ \\
\hline $\mathrm{C}(39)-\mathrm{C}(38)-\mathrm{C}(37)$ & $121.1(5)$ & $\mathrm{C}(51)-\mathrm{C}(50)-\mathrm{C}(46)$ & $119.5(4)$ \\
\hline $\mathrm{C}(38)-\mathrm{C}(39)-\mathrm{C}(31)$ & $119.9(5)$ & $\mathrm{C}(51)-\mathrm{C}(50)-\mathrm{C}(49)$ & $132.0(4)$ \\
\hline$C(54)-C(46)-C(50)$ & $119.8(4)$ & $C(46)-C(50)-C(49)$ & $108.6(4)$ \\
\hline $\mathrm{C}(54)-\mathrm{C}(46)-\mathrm{C}(47)$ & $131.3(4)$ & $\mathrm{C}(51)-\mathrm{C}(50)-\operatorname{Zr}(2)$ & $123.5(3)$ \\
\hline$C(50)-C(46)-C(47)$ & $108.8(4)$ & $C(46)-C(50)-\operatorname{Zr}(2)$ & $73.7(2)$ \\
\hline$C(54)-C(46)-\operatorname{Zr}(2)$ & $123.9(3)$ & $C(49)-C(50)-Z r(2)$ & $70.1(2)$ \\
\hline$C(50)-C(46)-\operatorname{Zr}(2)$ & $74.8(2)$ & $\mathrm{C}(52)-\mathrm{C}(51)-\mathrm{C}(50)$ & $119.2(5)$ \\
\hline$C(47)-C(46)-\operatorname{Zr}(2)$ & $70.8(2)$ & $\mathrm{C}(51)-\mathrm{C}(52)-\mathrm{C}(53)$ & $121.4(5)$ \\
\hline$C(48)-C(47)-C(46)$ & $104.6(4)$ & $\mathrm{C}(54)-\mathrm{C}(53)-\mathrm{C}(52)$ & $120.8(5)$ \\
\hline $\mathrm{C}(48)-\mathrm{C}(47)-\mathrm{Si}(7)$ & $121.7(3)$ & $\mathrm{C}(53)-\mathrm{C}(54)-\mathrm{C}(46)$ & $119.3(5)$ \\
\hline$C(46)-C(47)-S i(7)$ & $124.6(3)$ & & \\
\hline
\end{tabular}




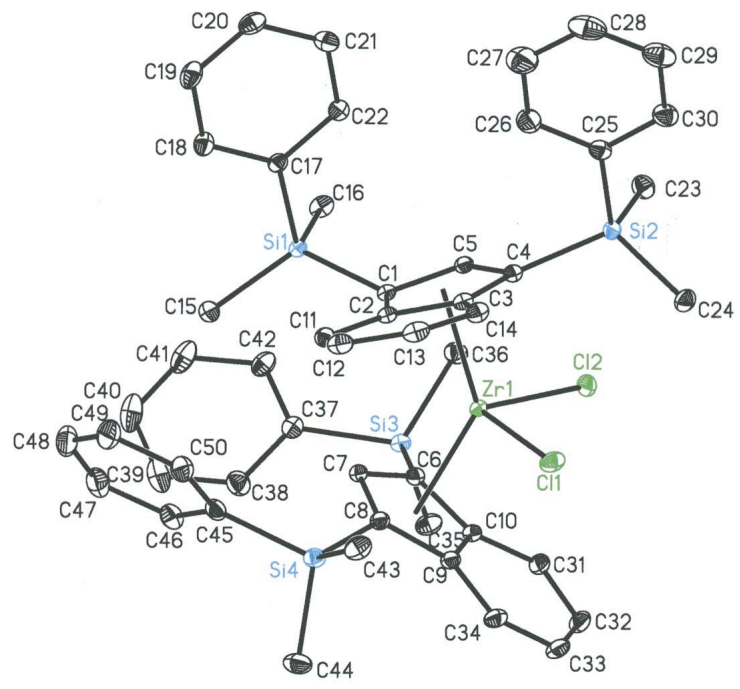

Figure S2. Atom labeling scheme for the solid state structure of $\mathbf{2}-\mathbf{C l}_{\mathbf{2}}$. 
Table S3. Crystal data and structure refinement for $\mathbf{2}-\mathbf{C l}_{\mathbf{2}}$.

Identification code

Empirical formula

Formula weight

Temperature

Wavelength

Crystal system

Space group

Unit cell dimensions

Volume

Z

Density (calculated)

Absorption coefficient

$\mathrm{F}(000)$

Crystal size

Theta range for data collection

Index ranges

Reflections collected

Independent reflections

Completeness to theta $=33.14^{\circ}$

Absorption correction

Max. and min. transmission

Refinement method

Data / restraints / parameters

Goodness-of-fit on $\mathrm{F}^{2}$

Final $\mathrm{R}$ indices $[\mathrm{I}>2 \operatorname{sigma}(\mathrm{I})]$

$\mathrm{R}$ indices (all data)

Largest diff. peak and hole
2-Cl

$\mathrm{C} 50 \mathrm{H} 54 \mathrm{Cl} 2 \mathrm{Si} 4 \mathrm{Zr} * \mathrm{O}(\mathrm{C} 2 \mathrm{H} 5) 2$

1003.53

173(2) K

$0.71073 \AA$

Triclinic

P-1

$\begin{array}{ll}\mathrm{a}=11.5765(6) \AA & \square=91.8080(10)^{\circ} . \\ \mathrm{b}=14.5860(8) \AA & \square=95.3770(10)^{\circ} . \\ \mathrm{c}=15.4520(8) \AA & \square=96.6950(10)^{\circ} .\end{array}$

2577.6(2) $\AA^{3}$

2

$1.293 \mathrm{Mg} / \mathrm{m}^{3}$

$0.447 \mathrm{~mm}^{-1}$

1052

$0.50 \times 0.30 \times 0.20 \mathrm{~mm}^{3}$

2.57 to $33.14^{\circ}$.

$-17<=\mathrm{h}<=16,-22<=\mathrm{k}<=22,-22<=1<=23$

54112

$18602[\mathrm{R}(\mathrm{int})=0.0353]$

$94.6 \%$

\section{SADABS}

0.9159 and 0.8074

Full-matrix least-squares on $\mathrm{F}^{2}$

18602 / 0 / 783

1.022

$\mathrm{R} 1=0.0393, \mathrm{wR} 2=0.0930$

$\mathrm{R} 1=0.0606, \mathrm{wR} 2=0.1016$

1.368 and -0.623 e. $\AA^{-3}$ 
Table S4. Bond lengths $[\AA]$ and angles $\left[{ }^{\circ}\right]$ for $\mathbf{2}-\mathbf{C l}_{\mathbf{2}}$.

\begin{tabular}{|c|c|c|c|}
\hline $\mathrm{Zr}(1)-\mathrm{Cl}(1)$ & $2.4130(4)$ & $C(4)-C(5)$ & $1.420(2)$ \\
\hline $\operatorname{Zr}(1)-\mathrm{Cl}(2)$ & $2.4330(4)$ & $\mathrm{C}(6)-\mathrm{C}(7)$ & $1.432(2)$ \\
\hline $\mathrm{Zr}(1)-\mathrm{C}(7)$ & $2.5029(15)$ & $C(6)-C(10)$ & $1.456(2)$ \\
\hline $\operatorname{Zr}(1)-C(5)$ & $2.5057(14)$ & $C(7)-C(8)$ & $1.423(2)$ \\
\hline $\operatorname{Zr}(1)-C(6)$ & $2.5126(16)$ & $\mathrm{C}(8)-\mathrm{C}(9)$ & $1.452(2)$ \\
\hline $\operatorname{Zr}(1)-C(4)$ & $2.5358(15)$ & $\mathrm{C}(9)-\mathrm{C}(34)$ & $1.425(2)$ \\
\hline $\mathrm{Zr}(1)-\mathrm{C}(1)$ & $2.5475(14)$ & $\mathrm{C}(9)-\mathrm{C}(10)$ & $1.426(2)$ \\
\hline $\mathrm{Zr}(1)-\mathrm{C}(8)$ & $2.5734(15)$ & $\mathrm{C}(10)-\mathrm{C}(31)$ & $1.426(2)$ \\
\hline $\mathrm{Zr}(1)-\mathrm{C}(2)$ & $2.6156(15)$ & $\mathrm{C}(11)-\mathrm{C}(12)$ & $1.368(2)$ \\
\hline $\operatorname{Zr}(1)-C(10)$ & $2.6217(15)$ & $C(12)-C(13)$ & $1.418(3)$ \\
\hline $\mathrm{Zr}(1)-\mathrm{C}(3)$ & $2.6399(15)$ & $C(13)-C(14)$ & $1.369(2)$ \\
\hline $\mathrm{Zr}(1)-\mathrm{C}(9)$ & $2.6621(15)$ & $C(17)-C(18)$ & $1.397(2)$ \\
\hline $\operatorname{Si}(1)-C(15)$ & $1.8603(17)$ & $\mathrm{C}(17)-\mathrm{C}(22)$ & $1.405(2)$ \\
\hline $\mathrm{Si}(1)-\mathrm{C}(16)$ & $1.8614(17)$ & $\mathrm{C}(18)-\mathrm{C}(19)$ & $1.394(3)$ \\
\hline $\mathrm{Si}(1)-\mathrm{C}(1)$ & $1.8759(15)$ & $C(19)-C(20)$ & $1.383(3)$ \\
\hline $\operatorname{Si}(1)-C(17)$ & $1.8837(16)$ & $C(20)-C(21)$ & $1.386(3)$ \\
\hline $\operatorname{Si}(2)-C(24)$ & $1.8583(18)$ & $C(21)-C(22)$ & $1.385(2)$ \\
\hline $\operatorname{Si}(2)-C(23)$ & $1.8587(18)$ & $C(25)-C(26)$ & $1.390(3)$ \\
\hline $\operatorname{Si}(2)-C(4)$ & $1.8756(16)$ & $C(25)-C(30)$ & $1.402(3)$ \\
\hline $\operatorname{Si}(2)-C(25)$ & $1.8875(18)$ & $C(26)-C(27)$ & $1.389(3)$ \\
\hline $\operatorname{Si}(3)-C(35)$ & $1.8642(18)$ & $\mathrm{C}(27)-\mathrm{C}(28)$ & $1.382(4)$ \\
\hline $\operatorname{Si}(3)-C(36)$ & $1.8700(17)$ & $C(28)-C(29)$ & $1.382(4)$ \\
\hline $\operatorname{Si}(3)-C(6)$ & $1.8803(16)$ & $C(29)-C(30)$ & $1.387(3)$ \\
\hline $\operatorname{Si}(3)-C(37)$ & $1.8830(17)$ & $\mathrm{C}(31)-\mathrm{C}(32)$ & $1.370(2)$ \\
\hline $\operatorname{Si}(4)-C(43)$ & $1.859(2)$ & $\mathrm{C}(32)-\mathrm{C}(33)$ & $1.413(3)$ \\
\hline $\operatorname{Si}(4)-C(44)$ & $1.869(2)$ & $\mathrm{C}(33)-\mathrm{C}(34)$ & $1.368(3)$ \\
\hline $\mathrm{Si}(4)-\mathrm{C}(8)$ & $1.8813(15)$ & $\mathrm{C}(37)-\mathrm{C}(42)$ & $1.397(2)$ \\
\hline $\operatorname{Si}(4)-C(45)$ & $1.8826(18)$ & $\mathrm{C}(37)-\mathrm{C}(38)$ & $1.401(2)$ \\
\hline$C(1)-C(5)$ & $1.430(2)$ & $\mathrm{C}(38)-\mathrm{C}(39)$ & $1.391(3)$ \\
\hline$C(1)-C(2)$ & $1.451(2)$ & $\mathrm{C}(39)-\mathrm{C}(40)$ & $1.379(4)$ \\
\hline $\mathrm{C}(2)-\mathrm{C}(11)$ & $1.424(2)$ & $\mathrm{C}(40)-\mathrm{C}(41)$ & $1.378(4)$ \\
\hline$C(2)-C(3)$ & $1.428(2)$ & $\mathrm{C}(41)-\mathrm{C}(42)$ & $1.397(3)$ \\
\hline $\mathrm{C}(3)-\mathrm{C}(14)$ & $1.424(2)$ & $\mathrm{C}(45)-\mathrm{C}(50)$ & $1.397(3)$ \\
\hline$C(3)-C(4)$ & $1.443(2)$ & $\mathrm{C}(45)-\mathrm{C}(46)$ & $1.400(3)$ \\
\hline
\end{tabular}




\begin{tabular}{|c|c|c|c|}
\hline $\mathrm{C}(46)-\mathrm{C}(47)$ & $1.395(3)$ & $C(1)-\operatorname{Zr}(1)-C(8)$ & $100.57(5)$ \\
\hline $\mathrm{C}(47)-\mathrm{C}(48)$ & $1.384(3)$ & $\mathrm{Cl}(1)-\mathrm{Zr}(1)-\mathrm{C}(2)$ & $99.62(4)$ \\
\hline C(48)-C(49) & $1.375(4)$ & $\mathrm{Cl}(2)-\mathrm{Zr}(1)-\mathrm{C}(2)$ & $132.77(3)$ \\
\hline $\mathrm{C}(49)-\mathrm{C}(50)$ & $1.391(3)$ & $C(7)-Z r(1)-C(2)$ & $87.93(5)$ \\
\hline $\mathrm{O}(1 \mathrm{~S})-\mathrm{C}(1 \mathrm{~S})$ & $1.384(5)$ & $C(5)-Z r(1)-C(2)$ & $52.73(5)$ \\
\hline $\mathrm{O}(1 \mathrm{~S})-\mathrm{C}(3 \mathrm{~S})$ & $1.411(4)$ & $C(6)-Z r(1)-C(2)$ & $114.99(5)$ \\
\hline $\mathrm{C}(1 \mathrm{~S})-\mathrm{C}(2 \mathrm{~S})$ & $1.444(7)$ & $C(4)-Z r(1)-C(2)$ & $53.84(5)$ \\
\hline \multirow[t]{2}{*}{$C(3 S)-C(4 S)$} & $1.448(5)$ & $C(1)-Z r(1)-C(2)$ & $32.62(5)$ \\
\hline & & $\mathrm{C}(8)-\mathrm{Zr}(1)-\mathrm{C}(2)$ & $91.55(5)$ \\
\hline $\mathrm{Cl}(1)-\mathrm{Zr}(1)-\mathrm{Cl}(2)$ & $97.178(16)$ & $\mathrm{Cl}(1)-\mathrm{Zr}(1)-\mathrm{C}(10)$ & $98.03(4)$ \\
\hline $\mathrm{Cl}(1)-\mathrm{Zr}(1)-\mathrm{C}(7)$ & $122.25(4)$ & $\mathrm{Cl}(2)-\mathrm{Zr}(1)-\mathrm{C}(10)$ & $78.78(3)$ \\
\hline $\mathrm{Cl}(2)-\mathrm{Zr}(1)-\mathrm{C}(7)$ & $118.68(4)$ & $C(7)-\operatorname{Zr}(1)-C(10)$ & $53.04(5)$ \\
\hline $\mathrm{Cl}(1)-\mathrm{Zr}(1)-\mathrm{C}(5)$ & $121.81(4)$ & $C(5)-\operatorname{Zr}(1)-C(10)$ & $137.13(5)$ \\
\hline $\mathrm{Cl}(2)-\mathrm{Zr}(1)-\mathrm{C}(5)$ & $81.00(4)$ & $C(6)-\operatorname{Zr}(1)-C(10)$ & $32.85(5)$ \\
\hline$C(7)-\operatorname{Zr}(1)-C(5)$ & $108.13(5)$ & $C(4)-\operatorname{Zr}(1)-C(10)$ & $160.94(5)$ \\
\hline $\mathrm{Cl}(1)-\mathrm{Zr}(1)-\mathrm{C}(6)$ & $129.45(4)$ & $C(1)-\operatorname{Zr}(1)-C(10)$ & $127.09(5)$ \\
\hline $\mathrm{Cl}(2)-\mathrm{Zr}(1)-\mathrm{C}(6)$ & $85.70(4)$ & $\mathrm{C}(8)-\operatorname{Zr}(1)-\mathrm{C}(10)$ & $53.61(5)$ \\
\hline$C(7)-\operatorname{Zr}(1)-C(6)$ & $33.18(5)$ & $C(2)-\operatorname{Zr}(1)-C(10)$ & $140.65(5)$ \\
\hline$C(5)-\operatorname{Zr}(1)-C(6)$ & $108.53(5)$ & $\mathrm{Cl}(1)-\mathrm{Zr}(1)-\mathrm{C}(3)$ & $77.52(3)$ \\
\hline $\mathrm{Cl}(1)-\mathrm{Zr}(1)-\mathrm{C}(4)$ & $89.12(4)$ & $\mathrm{Cl}(2)-\mathrm{Zr}(1)-\mathrm{C}(3)$ & $113.98(3)$ \\
\hline $\mathrm{Cl}(2)-\mathrm{Zr}(1)-\mathrm{C}(4)$ & $82.82(3)$ & $C(7)-\operatorname{Zr}(1)-C(3)$ & $118.75(5)$ \\
\hline$C(7)-\operatorname{Zr}(1)-C(4)$ & $135.64(5)$ & $C(5)-Z r(1)-C(3)$ & $52.40(5)$ \\
\hline$C(5)-\operatorname{Zr}(1)-C(4)$ & $32.72(5)$ & $C(6)-\operatorname{Zr}(1)-C(3)$ & $146.03(5)$ \\
\hline$C(6)-\operatorname{Zr}(1)-C(4)$ & $140.91(5)$ & $C(4)-\operatorname{Zr}(1)-C(3)$ & $32.30(5)$ \\
\hline $\mathrm{Cl}(1)-\mathrm{Zr}(1)-\mathrm{C}(1)$ & $130.39(4)$ & $C(1)-\operatorname{Zr}(1)-C(3)$ & $53.75(5)$ \\
\hline $\mathrm{Cl}(2)-\mathrm{Zr}(1)-\mathrm{C}(1)$ & 109.63(3) & $C(8)-\operatorname{Zr}(1)-C(3)$ & $113.51(5)$ \\
\hline $\mathrm{C}(7)-\operatorname{Zr}(1)-\mathrm{C}(1)$ & $80.23(5)$ & $C(2)-\operatorname{Zr}(1)-C(3)$ & $31.53(5)$ \\
\hline $\mathrm{C}(5)-\operatorname{Zr}(1)-\mathrm{C}(1)$ & $32.87(5)$ & $C(10)-\operatorname{Zr}(1)-C(3)$ & $166.76(5)$ \\
\hline$C(6)-\operatorname{Zr}(1)-C(1)$ & $94.41(5)$ & $\mathrm{Cl}(1)-\mathrm{Zr}(1)-\mathrm{C}(9)$ & $77.05(4)$ \\
\hline $\mathrm{C}(4)-\operatorname{Zr}(1)-\mathrm{C}(1)$ & $55.52(5)$ & $\mathrm{Cl}(2)-\mathrm{Zr}(1)-\mathrm{C}(9)$ & $104.21(4)$ \\
\hline $\mathrm{Cl}(1)-\mathrm{Zr}(1)-\mathrm{C}(8)$ & $89.78(4)$ & $C(7)-Z r(1)-C(9)$ & $52.38(5)$ \\
\hline $\mathrm{Cl}(2)-\mathrm{Zr}(1)-\mathrm{C}(8)$ & $132.39(4)$ & $C(5)-Z r(1)-C(9)$ & $160.12(5)$ \\
\hline$C(7)-\operatorname{Zr}(1)-C(8)$ & $32.53(5)$ & $C(6)-Z r(1)-C(9)$ & $53.71(5)$ \\
\hline$C(5)-\operatorname{Zr}(1)-C(8)$ & $133.18(5)$ & $C(4)-Z r(1)-C(9)$ & $165.07(5)$ \\
\hline$C(6)-\operatorname{Zr}(1)-C(8)$ & $55.43(5)$ & $C(1)-Z r(1)-C(9)$ & $131.02(5)$ \\
\hline $\mathrm{C}(4)-\operatorname{Zr}(1)-\mathrm{C}(8)$ & $144.56(5)$ & $\mathrm{C}(8)-\operatorname{Zr}(1)-\mathrm{C}(9)$ & $32.14(5)$ \\
\hline
\end{tabular}




\begin{tabular}{|c|c|c|c|}
\hline$C(2)-\operatorname{Zr}(1)-C(9)$ & $122.47(5)$ & $C(11)-C(2)-\operatorname{Zr}(1)$ & $126.24(10)$ \\
\hline$C(10)-\operatorname{Zr}(1)-C(9)$ & $31.30(5)$ & $C(3)-C(2)-\operatorname{Zr}(1)$ & $75.18(8)$ \\
\hline$C(3)-\operatorname{Zr}(1)-C(9)$ & $136.12(5)$ & $C(1)-C(2)-\operatorname{Zr}(1)$ & $71.11(8)$ \\
\hline$C(15)-\operatorname{Si}(1)-C(16)$ & $110.67(9)$ & $C(14)-C(3)-C(2)$ & $120.23(14)$ \\
\hline$C(15)-\operatorname{Si}(1)-C(1)$ & $116.54(7)$ & $\mathrm{C}(14)-\mathrm{C}(3)-\mathrm{C}(4)$ & $130.96(15)$ \\
\hline$C(16)-\operatorname{Si}(1)-C(1)$ & $110.91(8)$ & $C(2)-C(3)-C(4)$ & $108.71(13)$ \\
\hline $\mathrm{C}(15)-\mathrm{Si}(1)-\mathrm{C}(17)$ & $110.09(8)$ & $C(14)-C(3)-\operatorname{Zr}(1)$ & $125.65(10)$ \\
\hline $\mathrm{C}(16)-\mathrm{Si}(1)-\mathrm{C}(17)$ & $105.49(7)$ & $C(2)-C(3)-\operatorname{Zr}(1)$ & $73.30(8)$ \\
\hline$C(1)-\operatorname{Si}(1)-C(17)$ & $102.27(7)$ & $C(4)-C(3)-\operatorname{Zr}(1)$ & $69.88(8)$ \\
\hline $\mathrm{C}(24)-\mathrm{Si}(2)-\mathrm{C}(23)$ & 109.90(9) & $C(5)-C(4)-C(3)$ & $105.22(13)$ \\
\hline $\mathrm{C}(24)-\mathrm{Si}(2)-\mathrm{C}(4)$ & $113.93(8)$ & $C(5)-C(4)-S i(2)$ & $126.28(11)$ \\
\hline $\mathrm{C}(23)-\mathrm{Si}(2)-\mathrm{C}(4)$ & $109.24(8)$ & $C(3)-C(4)-S i(2)$ & $126.43(12)$ \\
\hline$C(24)-\operatorname{Si}(2)-C(25)$ & $109.75(8)$ & $C(5)-C(4)-\operatorname{Zr}(1)$ & $72.48(8)$ \\
\hline $\mathrm{C}(23)-\mathrm{Si}(2)-\mathrm{C}(25)$ & $108.70(8)$ & $C(3)-C(4)-\operatorname{Zr}(1)$ & $77.82(8)$ \\
\hline $\mathrm{C}(4)-\mathrm{Si}(2)-\mathrm{C}(25)$ & $105.12(7)$ & $\mathrm{Si}(2)-\mathrm{C}(4)-\operatorname{Zr}(1)$ & $127.42(7)$ \\
\hline $\mathrm{C}(35)-\mathrm{Si}(3)-\mathrm{C}(36)$ & $110.36(9)$ & $C(4)-C(5)-C(1)$ & $112.31(13)$ \\
\hline$C(35)-\operatorname{Si}(3)-C(6)$ & $108.14(8)$ & $C(4)-C(5)-\operatorname{Zr}(1)$ & $74.81(8)$ \\
\hline$C(36)-\operatorname{Si}(3)-C(6)$ & $113.67(8)$ & $C(1)-C(5)-\operatorname{Zr}(1)$ & $75.17(8)$ \\
\hline$C(35)-\operatorname{Si}(3)-C(37)$ & $106.46(9)$ & $C(7)-C(6)-C(10)$ & $104.98(13)$ \\
\hline $\mathrm{C}(36)-\mathrm{Si}(3)-\mathrm{C}(37)$ & $110.23(8)$ & $C(7)-C(6)-S i(3)$ & $126.09(11)$ \\
\hline C(6)-Si(3)-C(37) & $107.68(7)$ & $C(10)-C(6)-S i(3)$ & $125.62(11)$ \\
\hline C(43)-Si(4)-C(44) & $108.55(10)$ & $C(7)-C(6)-\operatorname{Zr}(1)$ & 73.04(9) \\
\hline $\mathrm{C}(43)-\mathrm{Si}(4)-\mathrm{C}(8)$ & 112.92(9) & $C(10)-C(6)-\operatorname{Zr}(1)$ & $77.70(9)$ \\
\hline C(44)-Si(4)-C(8) & $106.90(8)$ & $\operatorname{Si}(3)-C(6)-\operatorname{Zr}(1)$ & $130.33(7)$ \\
\hline $\mathrm{C}(43)-\mathrm{Si}(4)-\mathrm{C}(45)$ & $112.37(9)$ & $C(8)-C(7)-C(6)$ & $111.90(13)$ \\
\hline $\mathrm{C}(44)-\mathrm{Si}(4)-\mathrm{C}(45)$ & $106.46(9)$ & $C(8)-C(7)-\operatorname{Zr}(1)$ & $76.46(9)$ \\
\hline $\mathrm{C}(8)-\mathrm{Si}(4)-\mathrm{C}(45)$ & $109.30(7)$ & $C(6)-C(7)-\operatorname{Zr}(1)$ & $73.78(9)$ \\
\hline$C(5)-C(1)-C(2)$ & $104.36(13)$ & $\mathrm{C}(7)-\mathrm{C}(8)-\mathrm{C}(9)$ & $105.19(13)$ \\
\hline$C(5)-C(1)-S i(1)$ & $122.98(11)$ & $C(7)-C(8)-S i(4)$ & $127.34(11)$ \\
\hline$C(2)-C(1)-S i(1)$ & $121.41(11)$ & $C(9)-C(8)-S i(4)$ & $123.08(11)$ \\
\hline$C(5)-C(1)-\operatorname{Zr}(1)$ & $71.96(8)$ & $C(7)-C(8)-\operatorname{Zr}(1)$ & $71.01(8)$ \\
\hline $\mathrm{C}(2)-\mathrm{C}(1)-\mathrm{Zr}(1)$ & $76.27(8)$ & $\mathrm{C}(9)-\mathrm{C}(8)-\operatorname{Zr}(1)$ & $77.30(9)$ \\
\hline $\operatorname{Si}(1)-C(1)-\operatorname{Zr}(1)$ & $146.16(8)$ & $\operatorname{Si}(4)-C(8)-\operatorname{Zr}(1)$ & $135.12(8)$ \\
\hline $\mathrm{C}(11)-\mathrm{C}(2)-\mathrm{C}(3)$ & $119.72(14)$ & $C(34)-C(9)-C(10)$ & $120.05(15)$ \\
\hline$C(11)-C(2)-C(1)$ & $130.66(14)$ & $C(34)-C(9)-C(8)$ & $130.90(15)$ \\
\hline $\mathrm{C}(3)-\mathrm{C}(2)-\mathrm{C}(1)$ & $109.15(13)$ & $\mathrm{C}(10)-\mathrm{C}(9)-\mathrm{C}(8)$ & $109.04(13)$ \\
\hline
\end{tabular}




$\begin{array}{lc}\mathrm{C}(34)-\mathrm{C}(9)-\mathrm{Zr}(1) & 123.98(11) \\ \mathrm{C}(10)-\mathrm{C}(9)-\mathrm{Zr}(1) & 72.78(8) \\ \mathrm{C}(8)-\mathrm{C}(9)-\mathrm{Zr}(1) & 70.56(8) \\ \mathrm{C}(31)-\mathrm{C}(10)-\mathrm{C}(9) & 119.49(14) \\ \mathrm{C}(31)-\mathrm{C}(10)-\mathrm{C}(6) & 131.79(15) \\ \mathrm{C}(9)-\mathrm{C}(10)-\mathrm{C}(6) & 108.71(13) \\ \mathrm{C}(31)-\mathrm{C}(10)-\mathrm{Zr}(1) & 121.99(11) \\ \mathrm{C}(9)-\mathrm{C}(10)-\mathrm{Zr}(1) & 75.92(9) \\ \mathrm{C}(6)-\mathrm{C}(10)-\mathrm{Zr}(1) & 69.45(8) \\ \mathrm{C}(12)-\mathrm{C}(11)-\mathrm{C}(2) & 118.38(16) \\ \mathrm{C}(11)-\mathrm{C}(12)-\mathrm{C}(13) & 121.95(15) \\ \mathrm{C}(14)-\mathrm{C}(13)-\mathrm{C}(12) & 121.18(16) \\ \mathrm{C}(13)-\mathrm{C}(14)-\mathrm{C}(3) & 118.43(16) \\ \mathrm{C}(18)-\mathrm{C}(17)-\mathrm{C}(22) & 116.87(15) \\ \mathrm{C}(18)-\mathrm{C}(17)-\mathrm{Si}(1) & 124.02(13) \\ \mathrm{C}(22)-\mathrm{C}(17)-\mathrm{Si}(1) & 118.86(12) \\ \mathrm{C}(19)-\mathrm{C}(18)-\mathrm{C}(17) & 121.29(17) \\ \mathrm{C}(20)-\mathrm{C}(19)-\mathrm{C}(18) & 120.36(17) \\ \mathrm{C}(19)-\mathrm{C}(20)-\mathrm{C}(21) & 119.55(17) \\ \mathrm{C}(22)-\mathrm{C}(21)-\mathrm{C}(20) & 119.87(17) \\ \mathrm{C}(21)-\mathrm{C}(22)-\mathrm{C}(17) & 122.00(16) \\ \mathrm{C}(26)-\mathrm{C}(25)-\mathrm{C}(30) & 117.03(18) \\ \mathrm{C}(26)-\mathrm{C}(25)-\mathrm{Si}(2) & 123.04(14) \\ \mathrm{C}(30)-\mathrm{C}(25)-\mathrm{Si}(2) & 119.90(14) \\ \mathrm{C}(27)-\mathrm{C}(26)-\mathrm{C}(25) & 121.7(2) \\ \mathrm{C}(28)-\mathrm{C}(27)-\mathrm{C}(26) & 120.3(2) \\ \mathrm{C}(27)-\mathrm{C}(28)-\mathrm{C}(29) & 119.3(2) \\ \mathrm{C}(28)-\mathrm{C}(29)-\mathrm{C}(30) & 120.3(2)\end{array}$

$\begin{array}{ll}\mathrm{C}(29)-\mathrm{C}(30)-\mathrm{C}(25) & 121.4(2) \\ \mathrm{C}(32)-\mathrm{C}(31)-\mathrm{C}(10) & 118.95(16) \\ \mathrm{C}(31)-\mathrm{C}(32)-\mathrm{C}(33) & 121.22(16) \\ \mathrm{C}(34)-\mathrm{C}(33)-\mathrm{C}(32) & 121.66(16) \\ \mathrm{C}(33)-\mathrm{C}(34)-\mathrm{C}(9) & 118.55(16) \\ \mathrm{C}(42)-\mathrm{C}(37)-\mathrm{C}(38) & 117.13(16) \\ \mathrm{C}(42)-\mathrm{C}(37)-\mathrm{Si}(3) & 122.25(13) \\ \mathrm{C}(38)-\mathrm{C}(37)-\mathrm{Si}(3) & 120.34(13) \\ \mathrm{C}(39)-\mathrm{C}(38)-\mathrm{C}(37) & 121.38(19) \\ \mathrm{C}(40)-\mathrm{C}(39)-\mathrm{C}(38) & 120.2(2) \\ \mathrm{C}(41)-\mathrm{C}(40)-\mathrm{C}(39) & 119.8(2) \\ \mathrm{C}(40)-\mathrm{C}(41)-\mathrm{C}(42) & 120.0(2) \\ \mathrm{C}(37)-\mathrm{C}(42)-\mathrm{C}(41) & 121.43(19) \\ \mathrm{C}(50)-\mathrm{C}(45)-\mathrm{C}(46) & 117.29(17) \\ \mathrm{C}(50)-\mathrm{C}(45)-\mathrm{Si}(4) & 122.62(14) \\ \mathrm{C}(46)-\mathrm{C}(45)-\mathrm{Si}(4) & 120.03(14) \\ \mathrm{C}(47)-\mathrm{C}(46)-\mathrm{C}(45) & 121.39(19) \\ \mathrm{C}(48)-\mathrm{C}(47)-\mathrm{C}(46) & 119.8(2) \\ \mathrm{C}(49)-\mathrm{C}(48)-\mathrm{C}(47) & 119.8(2) \\ \mathrm{C}(48)-\mathrm{C}(49)-\mathrm{C}(50) & 120.4(2) \\ \mathrm{C}(49)-\mathrm{C}(50)-\mathrm{C}(45) & 121.3(2) \\ \mathrm{C}(1 \mathrm{~S})-\mathrm{O}(1 \mathrm{~S})-\mathrm{C}(3 \mathrm{~S}) & 115.7(3) \\ \mathrm{O}(1 \mathrm{~S})-\mathrm{C}(1 \mathrm{~S})-\mathrm{C}(2 \mathrm{~S}) & 111.0(4) \\ \mathrm{O}(1 \mathrm{~S})-\mathrm{C}(3 \mathrm{~S})-\mathrm{C}(4 \mathrm{~S}) & 108.7(3) \\ & \end{array}$

Symmetry transformations used to generate equivalent atoms: 


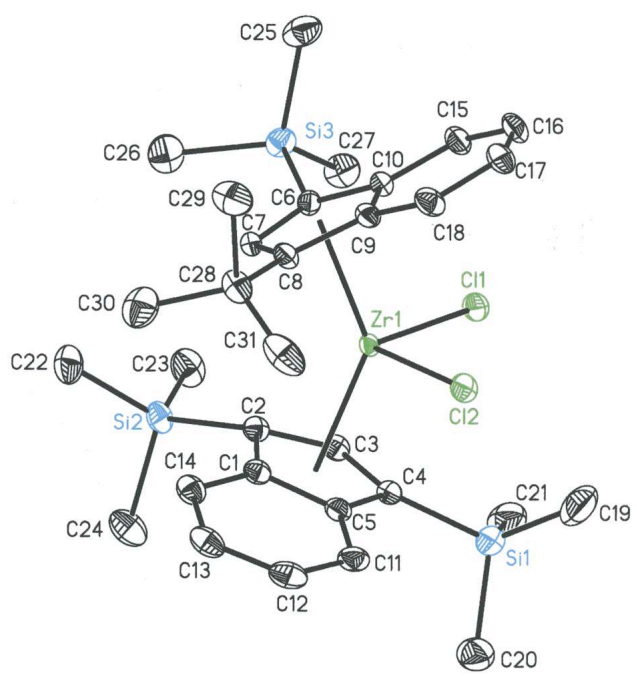

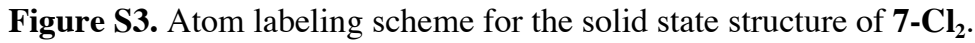




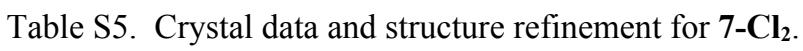

Identification code

Empirical formula

Formula weight

Temperature

Wavelength

Crystal system

Space group

Unit cell dimensions

Volume

Z

Density (calculated)

Absorption coefficient

$\mathrm{F}(000)$

Crystal size

Theta range for data collection

Index ranges

Reflections collected

Independent reflections

Completeness to theta $=30.51^{\circ}$

Absorption correction

Max. and min. transmission

Refinement method

Data / restraints / parameters

Goodness-of-fit on $\mathrm{F}^{2}$

Final $\mathrm{R}$ indices $[\mathrm{I}>2 \operatorname{sigma}(\mathrm{I})]$

$\mathrm{R}$ indices (all data)

Largest diff. peak and hole
7-Cl

C31 H46 C12 Si3 Zr

665.07

173(2) K

$0.71073 \AA$

Monoclinic

P2(1)/n

$\mathrm{a}=10.4644(2) \AA$

$\square=90^{\circ}$.

$\mathrm{b}=18.2115(4) \AA$

$\square=99.2770(10)^{\circ}$.

$\mathrm{c}=18.2169(3) \AA$

$\square=90^{\circ}$.

3426.23(11) $\AA^{3}$

4

$1.289 \mathrm{Mg} / \mathrm{m}^{3}$

$0.600 \mathrm{~mm}^{-1}$

1392

$0.50 \times 0.25 \times 0.10 \mathrm{~mm}^{3}$

1.59 to $30.51^{\circ}$.

$-14<=\mathrm{h}<=14,-26<=\mathrm{k}<=24,-26<=\mathrm{l}<=26$

52834

$10429[\mathrm{R}(\mathrm{int})=0.0597]$

$100.0 \%$

SADABS

0.9424 and 0.7534

Full-matrix least-squares on $\mathrm{F}^{2}$

10429 / 0 / 411

1.122

$\mathrm{R} 1=0.0584, \mathrm{wR} 2=0.1199$

$\mathrm{R} 1=0.0777, \mathrm{wR} 2=0.1278$

0.790 and -0.903 e. $\AA^{-3}$ 
Table S6. Bond lengths $[\AA]$ and angles $\left[{ }^{\circ}\right]$ for $\mathbf{7}-\mathbf{C l}_{\mathbf{2}}$.

\begin{tabular}{|c|c|c|c|}
\hline $\mathrm{Zr}(1)-\mathrm{Cl}(2)$ & $2.4176(7)$ & $\mathrm{C}(8)-\mathrm{C}(9)$ & $1.449(4)$ \\
\hline $\operatorname{Zr}(1)-\mathrm{Cl}(1)$ & $2.4275(8)$ & $\mathrm{C}(8)-\mathrm{C}(28)$ & $1.671(3)$ \\
\hline $\operatorname{Zr}(1)-C(3)$ & $2.488(3)$ & $C(9)-C(10)$ & $1.415(4)$ \\
\hline $\mathrm{Zr}(1)-\mathrm{C}(6)$ & $2.491(3)$ & $\mathrm{C}(9)-\mathrm{C}(18)$ & $1.425(4)$ \\
\hline $\mathrm{Zr}(1)-\mathrm{C}(7)$ & $2.512(3)$ & $C(10)-C(15)$ & $1.426(4)$ \\
\hline $\mathrm{Zr}(1)-\mathrm{C}(4)$ & $2.534(3)$ & $C(11)-C(12)$ & $1.361(5)$ \\
\hline $\mathrm{Zr}(1)-\mathrm{C}(2)$ & $2.548(3)$ & $C(12)-C(13)$ & $1.396(6)$ \\
\hline $\operatorname{Zr}(1)-C(10)$ & $2.600(3)$ & $C(13)-C(14)$ & $1.359(5)$ \\
\hline $\mathrm{Zr}(1)-\mathrm{C}(8)$ & $2.615(3)$ & $C(15)-C(16)$ & $1.363(5)$ \\
\hline $\operatorname{Zr}(1)-C(5)$ & $2.665(3)$ & $C(16)-C(17)$ & $1.406(6)$ \\
\hline $\operatorname{Zr}(1)-C(1)$ & $2.680(3)$ & $C(17)-C(18)$ & $1.364(5)$ \\
\hline $\operatorname{Zr}(1)-C(9)$ & $2.689(3)$ & $C(28)-C(31)$ & $1.504(7)$ \\
\hline $\operatorname{Si}(1)-C(19)$ & $1.800(6)$ & $C(28)-C(30)$ & $1.597(6)$ \\
\hline $\operatorname{Si}(1)-C(21)$ & $1.803(5)$ & $C(28)-C(29)$ & $1.664(5)$ \\
\hline $\operatorname{Si}(1)-C(20)$ & $1.829(4)$ & & \\
\hline $\operatorname{Si}(1)-C(4)$ & $1.842(3)$ & $\mathrm{Cl}(2)-\mathrm{Zr}(1)-\mathrm{Cl}(1)$ & $97.26(3)$ \\
\hline $\mathrm{Si}(2)-\mathrm{C}(24)$ & $1.850(4)$ & $\mathrm{Cl}(2)-\mathrm{Zr}(1)-\mathrm{C}(3)$ & $118.46(7)$ \\
\hline $\mathrm{Si}(2)-\mathrm{C}(23)$ & $1.855(4)$ & $\mathrm{Cl}(1)-\mathrm{Zr}(1)-\mathrm{C}(3)$ & $79.55(7)$ \\
\hline $\operatorname{Si}(2)-C(22)$ & $1.851(4)$ & $\mathrm{Cl}(2)-\mathrm{Zr}(1)-\mathrm{C}(6)$ & $128.13(7)$ \\
\hline $\mathrm{Si}(2)-\mathrm{C}(2)$ & $1.861(3)$ & $\mathrm{Cl}(1)-\mathrm{Zr}(1)-\mathrm{C}(6)$ & $86.09(7)$ \\
\hline $\operatorname{Si}(3)-C(25)$ & $1.842(4)$ & $C(3)-\operatorname{Zr}(1)-C(6)$ & $113.07(10)$ \\
\hline $\operatorname{Si}(3)-C(27)$ & $1.836(4)$ & $\mathrm{Cl}(2)-\mathrm{Zr}(1)-\mathrm{C}(7)$ & $120.70(7)$ \\
\hline $\mathrm{Si}(3)-\mathrm{C}(26)$ & $1.840(4)$ & $\mathrm{Cl}(1)-\mathrm{Zr}(1)-\mathrm{C}(7)$ & $119.12(7)$ \\
\hline $\operatorname{Si}(3)-C(6)$ & $1.851(3)$ & $C(3)-\operatorname{Zr}(1)-C(7)$ & $113.32(10)$ \\
\hline$C(1)-C(14)$ & $1.424(4)$ & $C(6)-\operatorname{Zr}(1)-C(7)$ & $33.19(9)$ \\
\hline $\mathrm{C}(1)-\mathrm{C}(5)$ & $1.426(4)$ & $\mathrm{Cl}(2)-\mathrm{Zr}(1)-\mathrm{C}(4)$ & $85.76(7)$ \\
\hline$C(1)-C(2)$ & $1.453(4)$ & $\mathrm{Cl}(1)-\mathrm{Zr}(1)-\mathrm{C}(4)$ & $83.98(7)$ \\
\hline $\mathrm{C}(2)-\mathrm{C}(3)$ & $1.424(4)$ & $C(3)-\operatorname{Zr}(1)-C(4)$ & $32.73(9)$ \\
\hline$C(3)-C(4)$ & $1.415(4)$ & $C(6)-\operatorname{Zr}(1)-C(4)$ & $145.69(10)$ \\
\hline $\mathrm{C}(4)-\mathrm{C}(5)$ & $1.438(4)$ & $C(7)-\operatorname{Zr}(1)-C(4)$ & $138.75(9)$ \\
\hline$C(5)-C(11)$ & $1.423(4)$ & $\mathrm{Cl}(2)-\mathrm{Zr}(1)-\mathrm{C}(2)$ & $130.01(7)$ \\
\hline$C(6)-C(7)$ & $1.429(4)$ & $\mathrm{Cl}(1)-\mathrm{Zr}(1)-\mathrm{C}(2)$ & $107.05(7)$ \\
\hline$C(6)-C(10)$ & $1.455(4)$ & $C(3)-\operatorname{Zr}(1)-C(2)$ & $32.81(10)$ \\
\hline$C(7)-C(8)$ & $1.415(4)$ & $C(6)-\operatorname{Zr}(1)-C(2)$ & $97.09(9)$ \\
\hline
\end{tabular}




\begin{tabular}{|c|c|c|c|}
\hline$C(7)-\operatorname{Zr}(1)-C(2)$ & $84.22(9)$ & $\mathrm{Cl}(2)-\mathrm{Zr}(1)-\mathrm{C}(9)$ & $75.76(7)$ \\
\hline $\mathrm{C}(4)-\mathrm{Zr}(1)-\mathrm{C}(2)$ & $55.31(9)$ & $\mathrm{Cl}(1)-\mathrm{Zr}(1)-\mathrm{C}(9)$ & $104.40(7)$ \\
\hline $\mathrm{Cl}(2)-\mathrm{Zr}(1)-\mathrm{C}(10)$ & $96.49(7)$ & $C(3)-\operatorname{Zr}(1)-C(9)$ & $165.02(9)$ \\
\hline $\mathrm{Cl}(1)-\mathrm{Zr}(1)-\mathrm{C}(10)$ & $79.20(7)$ & $C(6)-\operatorname{Zr}(1)-C(9)$ & $53.64(9)$ \\
\hline$C(3)-\operatorname{Zr}(1)-C(10)$ & $140.93(10)$ & $C(7)-\operatorname{Zr}(1)-C(9)$ & $51.99(9)$ \\
\hline$C(6)-\operatorname{Zr}(1)-C(10)$ & $33.12(9)$ & $C(4)-\operatorname{Zr}(1)-C(9)$ & $160.44(10)$ \\
\hline$C(7)-\operatorname{Zr}(1)-C(10)$ & $52.94(9)$ & $C(2)-\operatorname{Zr}(1)-C(9)$ & $134.79(9)$ \\
\hline$C(4)-\operatorname{Zr}(1)-C(10)$ & $163.18(9)$ & $C(10)-\operatorname{Zr}(1)-C(9)$ & $30.98(9)$ \\
\hline$C(2)-\operatorname{Zr}(1)-C(10)$ & $130.16(9)$ & $C(8)-\operatorname{Zr}(1)-C(9)$ & $31.67(9)$ \\
\hline $\mathrm{Cl}(2)-\mathrm{Zr}(1)-\mathrm{C}(8)$ & $88.79(7)$ & $C(5)-\operatorname{Zr}(1)-C(9)$ & $133.84(9)$ \\
\hline $\mathrm{Cl}(1)-\mathrm{Zr}(1)-\mathrm{C}(8)$ & $132.06(6)$ & $C(1)-\operatorname{Zr}(1)-C(9)$ & $123.81(9)$ \\
\hline $\mathrm{C}(3)-\operatorname{Zr}(1)-\mathrm{C}(8)$ & 137.41(9) & $C(19)-\operatorname{Si}(1)-C(21)$ & 110.1(3) \\
\hline $\mathrm{C}(6)-\mathrm{Zr}(1)-\mathrm{C}(8)$ & $54.87(9)$ & $C(19)-S i(1)-C(20)$ & $110.0(3)$ \\
\hline$C(7)-\operatorname{Zr}(1)-C(8)$ & $31.97(9)$ & $C(21)-\operatorname{Si}(1)-C(20)$ & $108.7(2)$ \\
\hline$C(4)-\operatorname{Zr}(1)-C(8)$ & $143.96(9)$ & $C(19)-S i(1)-C(4)$ & $115.14(19)$ \\
\hline$C(2)-\operatorname{Zr}(1)-C(8)$ & $104.63(9)$ & $C(21)-S i(1)-C(4)$ & 107.11(19) \\
\hline$C(10)-\operatorname{Zr}(1)-C(8)$ & $52.86(9)$ & $C(20)-S i(1)-C(4)$ & $105.58(18)$ \\
\hline $\mathrm{Cl}(2)-\mathrm{Zr}(1)-\mathrm{C}(5)$ & $76.96(7)$ & $C(24)-S i(2)-C(23)$ & $107.8(2)$ \\
\hline $\mathrm{Cl}(1)-\mathrm{Zr}(1)-\mathrm{C}(5)$ & $115.44(7)$ & $C(24)-S i(2)-C(22)$ & $107.8(2)$ \\
\hline $\mathrm{C}(3)-\operatorname{Zr}(1)-\mathrm{C}(5)$ & $52.07(10)$ & $C(23)-S i(2)-C(22)$ & $112.1(2)$ \\
\hline$C(6)-\operatorname{Zr}(1)-C(5)$ & $146.35(9)$ & $C(24)-S i(2)-C(2)$ & $103.26(17)$ \\
\hline$C(7)-\operatorname{Zr}(1)-C(5)$ & $118.17(9)$ & $C(23)-\mathrm{Si}(2)-\mathrm{C}(2)$ & $109.81(16)$ \\
\hline$C(4)-\operatorname{Zr}(1)-C(5)$ & $31.99(10)$ & $C(22)-S i(2)-C(2)$ & $115.40(16)$ \\
\hline$C(2)-\operatorname{Zr}(1)-C(5)$ & $53.25(9)$ & $C(25)-\operatorname{Si}(3)-C(27)$ & $108.10(19)$ \\
\hline$C(10)-\operatorname{Zr}(1)-C(5)$ & $164.38(10)$ & $C(25)-\operatorname{Si}(3)-C(26)$ & $107.9(2)$ \\
\hline$C(8)-\operatorname{Zr}(1)-C(5)$ & $112.26(9)$ & $C(27)-\operatorname{Si}(3)-C(26)$ & $112.0(2)$ \\
\hline $\mathrm{Cl}(2)-\mathrm{Zr}(1)-\mathrm{C}(1)$ & $100.73(6)$ & $C(25)-S i(3)-C(6)$ & $105.71(17)$ \\
\hline $\mathrm{Cl}(1)-\mathrm{Zr}(1)-\mathrm{C}(1)$ & $131.27(7)$ & $C(27)-S i(3)-C(6)$ & $114.40(16)$ \\
\hline$C(3)-\operatorname{Zr}(1)-C(1)$ & $52.07(9)$ & $C(26)-S i(3)-C(6)$ & $108.41(15)$ \\
\hline$C(6)-\operatorname{Zr}(1)-C(1)$ & $115.44(9)$ & $C(14)-C(1)-C(5)$ & $119.5(3)$ \\
\hline$C(7)-\operatorname{Zr}(1)-C(1)$ & $88.98(9)$ & $\mathrm{C}(14)-\mathrm{C}(1)-\mathrm{C}(2)$ & 131.3(3) \\
\hline$C(4)-\operatorname{Zr}(1)-C(1)$ & $53.05(9)$ & $C(5)-C(1)-C(2)$ & $108.6(3)$ \\
\hline$C(2)-\operatorname{Zr}(1)-C(1)$ & $32.15(9)$ & $C(14)-C(1)-\operatorname{Zr}(1)$ & $130.3(2)$ \\
\hline$C(10)-\operatorname{Zr}(1)-C(1)$ & $141.68(9)$ & $C(5)-C(1)-\operatorname{Zr}(1)$ & $73.94(16)$ \\
\hline$C(8)-\operatorname{Zr}(1)-C(1)$ & $93.39(9)$ & $C(2)-C(1)-\operatorname{Zr}(1)$ & $68.94(15)$ \\
\hline$C(5)-\operatorname{Zr}(1)-C(1)$ & $30.95(9)$ & $\mathrm{C}(3)-\mathrm{C}(2)-\mathrm{C}(1)$ & $104.5(2)$ \\
\hline
\end{tabular}




\begin{tabular}{|c|c|c|c|}
\hline $\mathrm{C}(3)-\mathrm{C}(2)-\mathrm{Si}(2)$ & $121.3(2)$ & $C(9)-C(8)-C(28)$ & $124.1(2)$ \\
\hline$C(1)-C(2)-S i(2)$ & $123.8(2)$ & $C(7)-C(8)-\operatorname{Zr}(1)$ & $70.00(17)$ \\
\hline$C(3)-C(2)-Z r(1)$ & $71.25(16)$ & $C(9)-C(8)-\operatorname{Zr}(1)$ & $76.99(17)$ \\
\hline$C(1)-C(2)-\operatorname{Zr}(1)$ & $78.91(16)$ & $C(28)-C(8)-\operatorname{Zr}(1)$ & $129.58(16)$ \\
\hline $\operatorname{Si}(2)-C(2)-\operatorname{Zr}(1)$ & $142.89(15)$ & $\mathrm{C}(10)-\mathrm{C}(9)-\mathrm{C}(18)$ & $119.5(3)$ \\
\hline $\mathrm{C}(4)-\mathrm{C}(3)-\mathrm{C}(2)$ & $112.4(3)$ & $C(10)-C(9)-C(8)$ & $108.3(3)$ \\
\hline$C(4)-C(3)-\operatorname{Zr}(1)$ & $75.42(17)$ & $C(18)-C(9)-C(8)$ & $132.2(3)$ \\
\hline$C(2)-C(3)-Z r(1)$ & $75.94(16)$ & $C(10)-C(9)-Z r(1)$ & $71.05(16)$ \\
\hline$C(3)-C(4)-C(5)$ & $105.2(3)$ & $C(18)-C(9)-\operatorname{Zr}(1)$ & $124.1(2)$ \\
\hline $\mathrm{C}(3)-\mathrm{C}(4)-\mathrm{Si}(1)$ & $125.0(2)$ & $C(8)-C(9)-\operatorname{Zr}(1)$ & $71.34(17)$ \\
\hline $\mathrm{C}(5)-\mathrm{C}(4)-\mathrm{Si}(1)$ & $128.0(2)$ & $C(9)-C(10)-C(15)$ & $119.9(3)$ \\
\hline$C(3)-C(4)-\operatorname{Zr}(1)$ & $71.85(16)$ & $C(9)-C(10)-C(6)$ & $109.5(2)$ \\
\hline$C(5)-C(4)-Z r(1)$ & $79.04(17)$ & $C(15)-C(10)-C(6)$ & $130.6(3)$ \\
\hline $\operatorname{Si}(1)-C(4)-\operatorname{Zr}(1)$ & $125.98(14)$ & $C(9)-C(10)-Z r(1)$ & $77.98(17)$ \\
\hline$C(11)-C(5)-C(1)$ & 119.3(3) & $C(15)-C(10)-\operatorname{Zr}(1)$ & $120.3(2)$ \\
\hline$C(11)-C(5)-C(4)$ & $131.6(3)$ & $C(6)-C(10)-Z r(1)$ & $69.31(15)$ \\
\hline$C(1)-C(5)-C(4)$ & $109.0(3)$ & $C(12)-C(11)-C(5)$ & $118.7(3)$ \\
\hline$C(11)-C(5)-\operatorname{Zr}(1)$ & $125.4(2)$ & $\mathrm{C}(11)-\mathrm{C}(12)-\mathrm{C}(13)$ & $121.9(3)$ \\
\hline$C(1)-C(5)-Z r(1)$ & $75.10(16)$ & $\mathrm{C}(14)-\mathrm{C}(13)-\mathrm{C}(12)$ & $121.5(3)$ \\
\hline$C(4)-C(5)-Z r(1)$ & $68.97(15)$ & $\mathrm{C}(13)-\mathrm{C}(14)-\mathrm{C}(1)$ & $118.9(3)$ \\
\hline$C(7)-C(6)-C(10)$ & $104.5(2)$ & $C(16)-C(15)-C(10)$ & $118.9(3)$ \\
\hline$C(7)-C(6)-S i(3)$ & $125.8(2)$ & $C(15)-C(16)-C(17)$ & $121.2(3)$ \\
\hline$C(10)-C(6)-\operatorname{Si}(3)$ & $124.0(2)$ & $\mathrm{C}(18)-\mathrm{C}(17)-\mathrm{C}(16)$ & $121.6(3)$ \\
\hline$C(7)-C(6)-Z r(1)$ & $74.20(16)$ & $C(17)-C(18)-C(9)$ & $118.9(3)$ \\
\hline$C(10)-C(6)-\operatorname{Zr}(1)$ & $77.56(16)$ & $\mathrm{C}(31)-\mathrm{C}(28)-\mathrm{C}(30)$ & $115.7(3)$ \\
\hline $\operatorname{Si}(3)-C(6)-\operatorname{Zr}(1)$ & $134.29(14)$ & $\mathrm{C}(31)-\mathrm{C}(28)-\mathrm{C}(29)$ & $111.6(3)$ \\
\hline $\mathrm{C}(8)-\mathrm{C}(7)-\mathrm{C}(6)$ & $111.8(2)$ & $\mathrm{C}(30)-\mathrm{C}(28)-\mathrm{C}(29)$ & $104.8(3)$ \\
\hline$C(8)-C(7)-\operatorname{Zr}(1)$ & $78.03(17)$ & $\mathrm{C}(31)-\mathrm{C}(28)-\mathrm{C}(8)$ & $112.7(2)$ \\
\hline$C(6)-C(7)-Z r(1)$ & $72.61(16)$ & $\mathrm{C}(30)-\mathrm{C}(28)-\mathrm{C}(8)$ & $108.1(3)$ \\
\hline $\mathrm{C}(7)-\mathrm{C}(8)-\mathrm{C}(9)$ & $105.8(2)$ & $\mathrm{C}(29)-\mathrm{C}(28)-\mathrm{C}(8)$ & $103.0(2)$ \\
\hline$C(7)-C(8)-C(28)$ & $128.3(2)$ & & \\
\hline
\end{tabular}




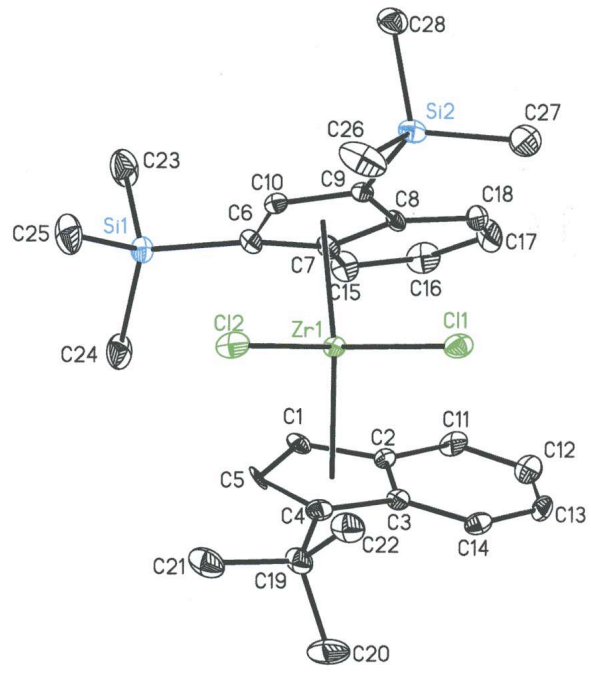

Figure S4. Atom labeling scheme for the solid state structure of $\mathbf{9}-\mathbf{C l}_{\mathbf{2}}$. 
Table S7. Crystal data and structure refinement for $\mathbf{9 -} \mathbf{C l}_{\mathbf{2}}$.

Identification code

Empirical formula

Formula weight

Temperature

Wavelength

Crystal system

Space group

Unit cell dimensions

Volume

Z

Density (calculated)

Absorption coefficient

$\mathrm{F}(000)$

Crystal size

Theta range for data collection

Index ranges

Reflections collected

Independent reflections

Completeness to theta $=23.25^{\circ}$

Absorption correction

Max. and min. transmission

Refinement method

Data / restraints / parameters

Goodness-of-fit on $\mathrm{F}^{2}$

Final $\mathrm{R}$ indices $[\mathrm{I}>2 \operatorname{sigma}(\mathrm{I})]$

$\mathrm{R}$ indices (all data)

Largest diff. peak and hole
9-Cl

C28 H38 C12 Si2 Zr

592.88

173(2) K

$0.71073 \AA$

Monoclinic

$\mathrm{P} 2(1) / \mathrm{c}$

$\mathrm{a}=9.4665(11) \AA$

$\square=90^{\circ}$.

$\mathrm{b}=16.131(2) \AA$

$\square=95.829(4)^{\circ}$.

$\mathrm{c}=19.225(3) \AA$

$\square=90^{\circ}$.
2920.6(7) $\AA^{3}$

4

$1.348 \mathrm{Mg} / \mathrm{m}^{3}$

$0.656 \mathrm{~mm}^{-1}$

1232

$0.4 \times 0.3 \times 0.2 \mathrm{~mm}^{3}$

1.65 to $23.25^{\circ}$.

$-9<=\mathrm{h}<=7,-17<=\mathrm{k}<=11,-21<=\mathrm{l}<=18$

9213

$3452[\mathrm{R}(\mathrm{int})=0.0740]$

$82.1 \%$

SADABS

0.921367 and 0.690517

Full-matrix least-squares on $\mathrm{F}^{2}$

3452 / 0 / 298

1.001

$\mathrm{R} 1=0.0501, \mathrm{wR} 2=0.1068$

$\mathrm{R} 1=0.0868, \mathrm{wR} 2=0.1212$

0.870 and -0.711 e. $\AA^{-3}$ 
Table S8. Bond lengths $[\AA]$ and angles $\left[{ }^{\circ}\right]$ for $\mathbf{9}-\mathbf{C l}_{\mathbf{2}}$.

\begin{tabular}{|c|c|c|c|}
\hline $\mathrm{Zr}(1)-\mathrm{Cl}(1)$ & $2.4111(18)$ & $\mathrm{C}(9)-\mathrm{C}(10)$ & $1.405(8)$ \\
\hline $\operatorname{Zr}(1)-C(1)$ & $2.427(5)$ & $C(11)-C(12)$ & $1.341(9)$ \\
\hline $\mathrm{Zr}(1)-\mathrm{Cl}(2)$ & $2.4312(17)$ & $C(12)-C(13)$ & $1.407(10)$ \\
\hline $\operatorname{Zr}(1)-C(10)$ & $2.500(6)$ & $C(13)-C(14)$ & $1.368(10)$ \\
\hline $\mathrm{Zr}(1)-\mathrm{C}(5)$ & $2.518(6)$ & $C(15)-C(16)$ & $1.355(9)$ \\
\hline $\operatorname{Zr}(1)-C(9)$ & $2.535(6)$ & $C(16)-C(17)$ & $1.402(9)$ \\
\hline $\mathrm{Zr}(1)-\mathrm{C}(6)$ & $2.538(6)$ & $C(17)-C(18)$ & $1.360(9)$ \\
\hline $\operatorname{Zr}(1)-C(2)$ & $2.563(5)$ & $C(19)-C(21)$ & $1.512(10)$ \\
\hline $\mathrm{Zr}(1)-\mathrm{C}(8)$ & $2.578(7)$ & $C(19)-C(22)$ & $1.551(9)$ \\
\hline $\operatorname{Zr}(1)-C(7)$ & $2.613(7)$ & $C(19)-C(20)$ & $1.562(9)$ \\
\hline $\operatorname{Zr}(1)-C(4)$ & $2.648(6)$ & & \\
\hline $\operatorname{Zr}(1)-C(3)$ & $2.694(6)$ & $\mathrm{Cl}(1)-\mathrm{Zr}(1)-\mathrm{C}(1)$ & $127.18(17)$ \\
\hline $\operatorname{Si}(1)-C(23)$ & $1.844(8)$ & $\mathrm{Cl}(1)-\mathrm{Zr}(1)-\mathrm{Cl}(2)$ & $98.27(7)$ \\
\hline $\mathrm{Si}(1)-\mathrm{C}(6)$ & $1.863(7)$ & $\mathrm{C}(1)-\mathrm{Zr}(1)-\mathrm{Cl}(2)$ & $113.24(18)$ \\
\hline $\operatorname{Si}(1)-C(24)$ & $1.863(6)$ & $\mathrm{Cl}(1)-\mathrm{Zr}(1)-\mathrm{C}(10)$ & $109.15(14)$ \\
\hline $\operatorname{Si}(1)-C(25)$ & $1.869(7)$ & $C(1)-\operatorname{Zr}(1)-C(10)$ & $118.7(2)$ \\
\hline $\operatorname{Si}(2)-C(27)$ & $1.841(8)$ & $\mathrm{Cl}(2)-\mathrm{Zr}(1)-\mathrm{C}(10)$ & $76.15(15)$ \\
\hline $\operatorname{Si}(2)-C(26)$ & $1.848(7)$ & $\mathrm{Cl}(1)-\mathrm{Zr}(1)-\mathrm{C}(5)$ & $123.22(17)$ \\
\hline $\operatorname{Si}(2)-C(28)$ & $1.856(7)$ & $C(1)-\operatorname{Zr}(1)-C(5)$ & $33.3(2)$ \\
\hline $\operatorname{Si}(2)-C(9)$ & $1.872(5)$ & $\mathrm{Cl}(2)-\mathrm{Zr}(1)-\mathrm{C}(5)$ & $82.86(16)$ \\
\hline$C(1)-C(2)$ & $1.414(9)$ & $C(10)-\operatorname{Zr}(1)-C(5)$ & $125.7(2)$ \\
\hline $\mathrm{C}(1)-\mathrm{C}(5)$ & $1.420(9)$ & $\mathrm{Cl}(1)-\operatorname{Zr}(1)-\mathrm{C}(9)$ & $80.90(15)$ \\
\hline C(2)-C(11) & $1.422(9)$ & $C(1)-\operatorname{Zr}(1)-C(9)$ & $132.9(2)$ \\
\hline$C(2)-C(3)$ & $1.432(9)$ & $\mathrm{Cl}(2)-\mathrm{Zr}(1)-\mathrm{C}(9)$ & $95.46(15)$ \\
\hline$C(3)-C(14)$ & $1.403(9)$ & $C(10)-\operatorname{Zr}(1)-C(9)$ & $32.41(19)$ \\
\hline$C(3)-C(4)$ & 1.431(9) & $C(5)-\operatorname{Zr}(1)-C(9)$ & $155.9(2)$ \\
\hline$C(4)-C(5)$ & $1.424(9)$ & $\mathrm{Cl}(1)-\mathrm{Zr}(1)-\mathrm{C}(6)$ & $136.62(14)$ \\
\hline C(4)-C(19) & $1.530(10)$ & $C(1)-\operatorname{Zr}(1)-C(6)$ & $85.5(2)$ \\
\hline$C(6)-C(10)$ & $1.442(7)$ & $\mathrm{Cl}(2)-\mathrm{Zr}(1)-\mathrm{C}(6)$ & $91.47(15)$ \\
\hline$C(6)-C(7)$ & $1.442(9)$ & $C(10)-\operatorname{Zr}(1)-C(6)$ & $33.27(17)$ \\
\hline$C(7)-C(8)$ & $1.421(8)$ & $C(5)-\operatorname{Zr}(1)-C(6)$ & $99.8(2)$ \\
\hline$C(7)-C(15)$ & $1.448(8)$ & $C(9)-\operatorname{Zr}(1)-C(6)$ & $56.05(19)$ \\
\hline$C(8)-C(18)$ & $1.418(9)$ & $\mathrm{Cl}(1)-\mathrm{Zr}(1)-\mathrm{C}(2)$ & $95.31(16)$ \\
\hline$C(8)-C(9)$ & $1.462(8)$ & $C(1)-\operatorname{Zr}(1)-C(2)$ & $32.8(2)$ \\
\hline
\end{tabular}




\begin{tabular}{|c|c|c|c|}
\hline $\mathrm{Cl}(2)-\mathrm{Zr}(1)-\mathrm{C}(2)$ & $134.09(16)$ & $C(5)-\operatorname{Zr}(1)-C(3)$ & $52.1(2)$ \\
\hline$C(10)-Z r(1)-C(2)$ & $138.4(2)$ & $C(9)-\operatorname{Zr}(1)-C(3)$ & $146.4(2)$ \\
\hline $\mathrm{C}(5)-\operatorname{Zr}(1)-\mathrm{C}(2)$ & $53.5(2)$ & $C(6)-\operatorname{Zr}(1)-C(3)$ & $137.65(17)$ \\
\hline $\mathrm{C}(9)-\operatorname{Zr}(1)-\mathrm{C}(2)$ & $130.0(2)$ & $C(2)-\operatorname{Zr}(1)-C(3)$ & $31.49(19)$ \\
\hline$C(6)-\operatorname{Zr}(1)-C(2)$ & $107.7(2)$ & $\mathrm{C}(8)-\operatorname{Zr}(1)-\mathrm{C}(3)$ & $120.3(2)$ \\
\hline $\mathrm{Cl}(1)-\mathrm{Zr}(1)-\mathrm{C}(8)$ & $86.92(15)$ & $C(7)-\operatorname{Zr}(1)-C(3)$ & $117.76(19)$ \\
\hline $\mathrm{C}(1)-\operatorname{Zr}(1)-\mathrm{C}(8)$ & $104.0(2)$ & $C(4)-\operatorname{Zr}(1)-C(3)$ & $31.0(2)$ \\
\hline $\mathrm{Cl}(2)-\mathrm{Zr}(1)-\mathrm{C}(8)$ & $127.17(14)$ & $C(23)-S i(1)-C(6)$ & 109.2(3) \\
\hline$C(10)-\operatorname{Zr}(1)-C(8)$ & $53.0(2)$ & $C(23)-S i(1)-C(24)$ & $107.4(4)$ \\
\hline $\mathrm{C}(5)-\operatorname{Zr}(1)-\mathrm{C}(8)$ & $136.2(2)$ & $C(6)-S i(1)-C(24)$ & 113.3(3) \\
\hline $\mathrm{C}(9)-\operatorname{Zr}(1)-\mathrm{C}(8)$ & $33.22(18)$ & $\mathrm{C}(23)-\mathrm{Si}(1)-\mathrm{C}(25)$ & $109.2(4)$ \\
\hline $\mathrm{C}(6)-\operatorname{Zr}(1)-\mathrm{C}(8)$ & $54.8(2)$ & $C(6)-S i(1)-C(25)$ & 108.1(3) \\
\hline $\mathrm{C}(2)-\mathrm{Zr}(1)-\mathrm{C}(8)$ & $97.1(2)$ & $\mathrm{C}(24)-\mathrm{Si}(1)-\mathrm{C}(25)$ & 109.6(3) \\
\hline $\mathrm{Cl}(1)-\mathrm{Zr}(1)-\mathrm{C}(7)$ & $117.81(15)$ & $\mathrm{C}(27)-\mathrm{Si}(2)-\mathrm{C}(26)$ & $111.4(4)$ \\
\hline$C(1)-\operatorname{Zr}(1)-C(7)$ & $79.0(2)$ & $\mathrm{C}(27)-\mathrm{Si}(2)-\mathrm{C}(28)$ & $105.5(3)$ \\
\hline $\mathrm{Cl}(2)-\mathrm{Zr}(1)-\mathrm{C}(7)$ & $123.40(15)$ & $C(26)-\operatorname{Si}(2)-C(28)$ & $110.6(4)$ \\
\hline$C(10)-Z r(1)-C(7)$ & $52.31(19)$ & $\mathrm{C}(27)-\mathrm{Si}(2)-\mathrm{C}(9)$ & $115.0(3)$ \\
\hline$C(5)-\operatorname{Zr}(1)-C(7)$ & $107.3(2)$ & $C(26)-S i(2)-C(9)$ & 109.2(3) \\
\hline $\mathrm{C}(9)-\operatorname{Zr}(1)-\mathrm{C}(7)$ & $53.91(17)$ & $\mathrm{C}(28)-\mathrm{Si}(2)-\mathrm{C}(9)$ & $105.0(3)$ \\
\hline$C(6)-\operatorname{Zr}(1)-C(7)$ & $32.47(19)$ & $C(2)-C(1)-C(5)$ & $107.5(6)$ \\
\hline$C(2)-\operatorname{Zr}(1)-C(7)$ & $86.7(2)$ & $C(2)-C(1)-\operatorname{Zr}(1)$ & $78.9(3)$ \\
\hline$C(8)-\operatorname{Zr}(1)-C(7)$ & $31.77(18)$ & $C(5)-C(1)-\operatorname{Zr}(1)$ & $76.8(3)$ \\
\hline $\mathrm{Cl}(1)-\mathrm{Zr}(1)-\mathrm{C}(4)$ & $91.52(16)$ & $C(1)-C(2)-C(11)$ & $132.3(7)$ \\
\hline $\mathrm{C}(1)-\operatorname{Zr}(1)-\mathrm{C}(4)$ & $54.1(2)$ & $\mathrm{C}(1)-\mathrm{C}(2)-\mathrm{C}(3)$ & $108.3(6)$ \\
\hline $\mathrm{Cl}(2)-\mathrm{Zr}(1)-\mathrm{C}(4)$ & $83.18(17)$ & $\mathrm{C}(11)-\mathrm{C}(2)-\mathrm{C}(3)$ & $119.3(7)$ \\
\hline$C(10)-Z r(1)-C(4)$ & $152.5(2)$ & $C(1)-C(2)-\operatorname{Zr}(1)$ & $68.3(3)$ \\
\hline$C(5)-\operatorname{Zr}(1)-C(4)$ & $31.9(2)$ & $\mathrm{C}(11)-\mathrm{C}(2)-\mathrm{Zr}(1)$ & $121.3(4)$ \\
\hline $\mathrm{C}(9)-\operatorname{Zr}(1)-\mathrm{C}(4)$ & $172.0(2)$ & $C(3)-C(2)-\operatorname{Zr}(1)$ & $79.3(3)$ \\
\hline$C(6)-\operatorname{Zr}(1)-C(4)$ & $131.7(2)$ & $\mathrm{C}(14)-\mathrm{C}(3)-\mathrm{C}(4)$ & $132.4(7)$ \\
\hline$C(2)-\operatorname{Zr}(1)-C(4)$ & $52.7(2)$ & $C(14)-C(3)-C(2)$ & $119.6(7)$ \\
\hline$C(8)-\operatorname{Zr}(1)-C(4)$ & $149.5(2)$ & $C(4)-C(3)-C(2)$ & $108.0(6)$ \\
\hline$C(7)-\operatorname{Zr}(1)-C(4)$ & $133.04(18)$ & $C(14)-C(3)-\operatorname{Zr}(1)$ & $124.9(4)$ \\
\hline $\mathrm{Cl}(1)-\mathrm{Zr}(1)-\mathrm{C}(3)$ & $76.38(14)$ & $C(4)-C(3)-\operatorname{Zr}(1)$ & $72.7(4)$ \\
\hline $\mathrm{C}(1)-\operatorname{Zr}(1)-\mathrm{C}(3)$ & $53.3(2)$ & $C(2)-C(3)-\operatorname{Zr}(1)$ & $69.2(3)$ \\
\hline $\mathrm{Cl}(2)-\mathrm{Zr}(1)-\mathrm{C}(3)$ & $111.93(16)$ & $\mathrm{C}(3)-\mathrm{C}(4)-\mathrm{C}(5)$ & $106.9(6)$ \\
\hline$C(10)-\operatorname{Zr}(1)-C(3)$ & $169.90(19)$ & $\mathrm{C}(3)-\mathrm{C}(4)-\mathrm{C}(19)$ & $127.7(6)$ \\
\hline
\end{tabular}




\begin{tabular}{|c|c|c|c|}
\hline$C(5)-C(4)-C(19)$ & $124.4(7)$ & $\mathrm{C}(10)-\mathrm{C}(9)-\mathrm{C}(8)$ & $104.5(5)$ \\
\hline$C(3)-C(4)-\operatorname{Zr}(1)$ & $76.2(4)$ & $C(10)-C(9)-S i(2)$ & $123.7(4)$ \\
\hline$C(5)-C(4)-\operatorname{Zr}(1)$ & $69.0(4)$ & $\mathrm{C}(8)-\mathrm{C}(9)-\mathrm{Si}(2)$ & $130.4(5)$ \\
\hline$C(19)-C(4)-\operatorname{Zr}(1)$ & $128.9(4)$ & $C(10)-C(9)-Z r(1)$ & $72.4(3)$ \\
\hline$C(1)-C(5)-C(4)$ & 109.2(6) & $C(8)-C(9)-\operatorname{Zr}(1)$ & $75.0(3)$ \\
\hline$C(1)-C(5)-\operatorname{Zr}(1)$ & $69.9(3)$ & $\mathrm{Si}(2)-\mathrm{C}(9)-\operatorname{Zr}(1)$ & $126.8(3)$ \\
\hline$C(4)-C(5)-\operatorname{Zr}(1)$ & 79.1(4) & $C(9)-C(10)-C(6)$ & $113.6(5)$ \\
\hline$C(10)-C(6)-C(7)$ & $102.9(5)$ & $C(9)-C(10)-Z r(1)$ & $75.2(4)$ \\
\hline$C(10)-C(6)-\operatorname{Si}(1)$ & $125.9(5)$ & $C(6)-C(10)-Z r(1)$ & $74.8(3)$ \\
\hline$C(7)-C(6)-S i(1)$ & $128.8(4)$ & $C(12)-C(11)-C(2)$ & $118.9(7)$ \\
\hline$C(10)-C(6)-\operatorname{Zr}(1)$ & $71.9(3)$ & $C(11)-C(12)-C(13)$ & $122.2(8)$ \\
\hline$C(7)-C(6)-Z r(1)$ & $76.6(4)$ & $\mathrm{C}(14)-\mathrm{C}(13)-\mathrm{C}(12)$ & $120.7(7)$ \\
\hline $\operatorname{Si}(1)-C(6)-\operatorname{Zr}(1)$ & $128.8(3)$ & $C(13)-C(14)-C(3)$ & $119.2(7)$ \\
\hline$C(8)-C(7)-C(6)$ & $110.5(5)$ & $C(16)-C(15)-C(7)$ & $118.4(6)$ \\
\hline$C(8)-C(7)-C(15)$ & $118.3(6)$ & $C(15)-C(16)-C(17)$ & $122.5(6)$ \\
\hline$C(6)-C(7)-C(15)$ & $130.9(6)$ & $C(18)-C(17)-C(16)$ & $120.9(7)$ \\
\hline$C(8)-C(7)-\operatorname{Zr}(1)$ & $72.7(4)$ & $C(17)-C(18)-C(8)$ & $119.1(6)$ \\
\hline$C(6)-C(7)-Z r(1)$ & $70.9(4)$ & $C(21)-C(19)-C(4)$ & $112.3(6)$ \\
\hline$C(15)-C(7)-\operatorname{Zr}(1)$ & $127.7(4)$ & $C(21)-C(19)-C(22)$ & $110.1(6)$ \\
\hline$C(7)-C(8)-C(18)$ & $120.5(5)$ & $C(4)-C(19)-C(22)$ & $110.9(6)$ \\
\hline $\mathrm{C}(7)-\mathrm{C}(8)-\mathrm{C}(9)$ & $108.1(6)$ & $C(21)-C(19)-C(20)$ & $108.2(6)$ \\
\hline $\mathrm{C}(18)-\mathrm{C}(8)-\mathrm{C}(9)$ & 131.1(5) & $C(4)-C(19)-C(20)$ & $107.6(6)$ \\
\hline$C(7)-C(8)-\operatorname{Zr}(1)$ & $75.5(4)$ & $\mathrm{C}(22)-\mathrm{C}(19)-\mathrm{C}(20)$ & $107.7(6)$ \\
\hline$C(18)-C(8)-\operatorname{Zr}(1)$ & $123.8(4)$ & & \\
\hline $\mathrm{C}(9)-\mathrm{C}(8)-\mathrm{Zr}(1)$ & $71.8(4)$ & & \\
\hline
\end{tabular}




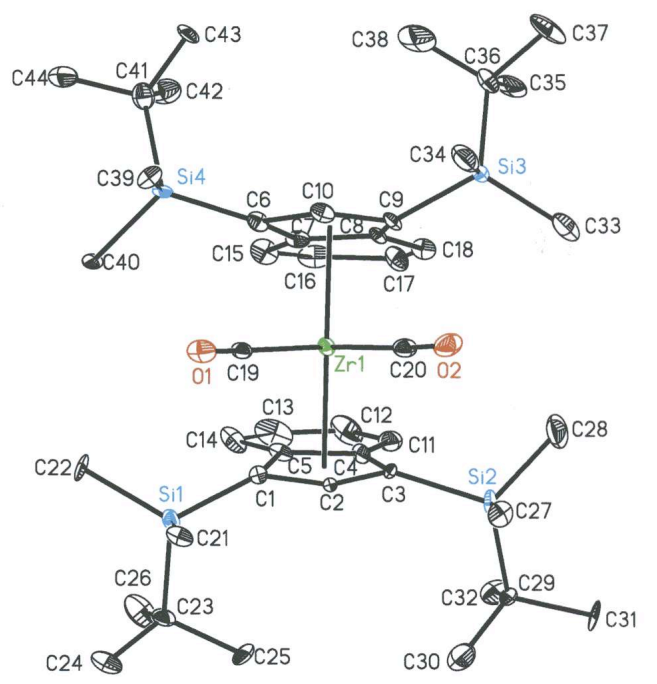

Figure S5. Atom labeling scheme for the solid state structure of 3-(CO) $)_{2}$. 
Table S9. Crystal data and structure refinement for $\mathbf{3}-(\mathbf{C O})_{2}$.

Identification code

Empirical formula

Formula weight

Temperature

Wavelength

Crystal system

Space group

Unit cell dimensions

Volume

Z

Density (calculated)

Absorption coefficient

$\mathrm{F}(000)$

Crystal size

Theta range for data collection

Index ranges

Reflections collected

Independent reflections

Completeness to theta $=30.51^{\circ}$

Absorption correction

Max. and min. transmission

Refinement method

Data / restraints / parameters

Goodness-of-fit on $\mathrm{F}^{2}$

Final $\mathrm{R}$ indices $[\mathrm{I}>2 \operatorname{sigma}(\mathrm{I})]$

$\mathrm{R}$ indices (all data)

Absolute structure parameter

Largest diff. peak and hole

\section{3-(CO)2}

C44 H70 O2 Si4 Zr

834.58

173(2) K

$0.71073 \AA$

Monoclinic

Cc
$a=20.7006(9) \AA$
$\square=90^{\circ}$.
$\mathrm{b}=9.3584(4) \AA$
$\square=96.702(1)^{\circ}$.
$\mathrm{c}=23.7365(11) \AA$
$\square=90^{\circ}$.

4566.9(3) $\AA^{3}$

4

$1.214 \mathrm{Mg} / \mathrm{m}^{3}$

$0.379 \mathrm{~mm}^{-1}$

1784

$0.35 \times 0.20 \times 0.10 \mathrm{~mm}^{3}$

2.39 to $30.51^{\circ}$.

$-27<=\mathrm{h}<=29,-13<=\mathrm{k}<=13,-33<=\mathrm{l}<=18$

16250

$9214[\mathrm{R}($ int $)=0.0402]$

$97.1 \%$

\section{SADABS}

0.9631 and 0.8788

Full-matrix least-squares on $\mathrm{F}^{2}$

9214 / 2 / 471

0.988

$\mathrm{R} 1=0.0405, \mathrm{wR} 2=0.0790$

$\mathrm{R} 1=0.0742, \mathrm{wR} 2=0.0902$

$0.67(9)$

0.402 and -0.300 e. $\AA^{-3}$ 
Table S10. Bond lengths $[\AA]$ and angles $\left[^{\circ}\right]$ for 3-(CO) $)_{2}$.

$\operatorname{Zr}(1)-C(20)$

2.133(10)

$\mathrm{Zr}(1)-\mathrm{C}(19)$

2.195(9)

$\mathrm{C}(4)-\mathrm{C}(5)$

1.435(12)

$\operatorname{Zr}(1)-\mathrm{C}(10)$

2.454(9)

C(4)-C(11)

1.434(11)

$\mathrm{Zr}(1)-\mathrm{C}(3)$

2.516(9)

C(5)-C(14)

1.374(12)

$\operatorname{Zr}(1)-\mathrm{C}(9)$

2.532(9)

$\mathrm{C}(6)-\mathrm{C}(10)$

1.447(12)

$\mathrm{Zr}(1)-\mathrm{C}(2)$

2.535(8)

$\mathrm{C}(6)-\mathrm{C}(7)$

1.502(12)

$\mathrm{Zr}(1)-\mathrm{C}(5)$

2.543(8)

$\operatorname{Zr}(1)-\mathrm{C}(4)$

2.547(8)

$\mathrm{Zr}(1)-\mathrm{C}(7)$

2.566(9)

$\mathrm{Zr}(1)-\mathrm{C}(8)$

2.584(8)

$\mathrm{C}(7)-\mathrm{C}(15)$

1.432(11)

$\mathrm{C}(7)-\mathrm{C}(8)$

1.454(12)

$\mathrm{C}(8)-\mathrm{C}(9)$

1.348(11)

C(8)-C(18)

1.483(10)

$\mathrm{C}(9)-\mathrm{C}(10)$

1.401(13)

$\mathrm{Zr}(1)-\mathrm{C}(6)$

2.570(10)

$\mathrm{C}(11)-\mathrm{C}(12)$

1.328(14)

2.592(9)

C(12)-C(13)

1.305(16)

1.847(9)

C(13)-C(14)

1.488(13)

1.878(8)

C(15)-C(16)

1.399(13)

1.867(6)

C(16)-C(17)

1.483(15)

1.946(10)

$\mathrm{C}(17)-\mathrm{C}(18)$

1.255(13)

1.863(9)

$\mathrm{C}(23)-\mathrm{C}(24)$

1.486(14)

1.889(7)

$1.550(14)$

1.903(9)

$\mathrm{C}(23)-\mathrm{C}(26)$

1.562(13)

1.927(9)

$\mathrm{C}(23)-\mathrm{C}(25)$

1.460(14)

1.859(10)

C(29)-C(32)

1.522(14)

1.864(11)

$\mathrm{C}(29)-\mathrm{C}(30)$

1.606(13)

1.843(10)

$\mathrm{C}(29)-\mathrm{C}(31)$

1.489(15)

1.910(9)

$\mathrm{C}(35)-\mathrm{C}(38)$

1.515(14)

1.815(10)

$\mathrm{C}(35)-\mathrm{C}(36)$

1.587(12)

1.837(10)

1.473(15)

$1.875(6)$

1.532(13)

1.905(11)

1.613(13)

$\mathrm{O}(1)-\mathrm{C}(19)$

1.123(10)

$\mathrm{O}(2)-\mathrm{C}(20)$

1.172(12)

1.462(11)

$\mathrm{C}(35)-\mathrm{C}(37)$

C(41)-C(44)

$\mathrm{C}(41)-\mathrm{C}(43)$

$\mathrm{C}(1)-\mathrm{C}(2)$

$1.516(10)$

$\mathrm{C}(41)-\mathrm{C}(42)$

84.59(11)

$\mathrm{C}(1)-\mathrm{C}(5)$

$\mathrm{C}(20)-\operatorname{Zr}(1)-\mathrm{C}(19)$

82.4(3)

$\mathrm{C}(20)-\operatorname{Zr}(1)-\mathrm{C}(10)$

81.5(3)

$\mathrm{C}(2)-\mathrm{C}(3)$

1.421(11)

$\mathrm{C}(19)-\operatorname{Zr}(1)-\mathrm{C}(10)$

$77.5(3)$

$\mathrm{C}(3)-\mathrm{C}(4)$

1.396(12)

$\mathrm{C}(20)-\mathrm{Zr}(1)-\mathrm{C}(3)$

112.0(3) 


\begin{tabular}{|c|c|c|c|}
\hline$C(10)-\operatorname{Zr}(1)-C(3)$ & $154.4(3)$ & $C(2)-\operatorname{Zr}(1)-C(8)$ & $149.5(3)$ \\
\hline$C(20)-\operatorname{Zr}(1)-C(9)$ & $74.2(3)$ & $C(5)-\operatorname{Zr}(1)-C(8)$ & 111.61(11) \\
\hline$C(19)-\operatorname{Zr}(1)-C(9)$ & 111.7(3) & $C(4)-\operatorname{Zr}(1)-C(8)$ & $99.4(3)$ \\
\hline$C(10)-\operatorname{Zr}(1)-C(9)$ & $32.6(3)$ & $C(7)-\operatorname{Zr}(1)-C(8)$ & $32.8(3)$ \\
\hline $\mathrm{C}(3)-\operatorname{Zr}(1)-\mathrm{C}(9)$ & $124.5(3)$ & $C(20)-\operatorname{Zr}(1)-C(6)$ & $114.9(3)$ \\
\hline$C(20)-Z r(1)-C(2)$ & $78.3(3)$ & $C(19)-\operatorname{Zr}(1)-C(6)$ & $78.1(3)$ \\
\hline$C(19)-\operatorname{Zr}(1)-C(2)$ & $79.6(3)$ & $C(10)-\operatorname{Zr}(1)-C(6)$ & $33.4(3)$ \\
\hline$C(10)-Z r(1)-C(2)$ & $154.10(10)$ & $C(3)-\operatorname{Zr}(1)-C(6)$ & $165.41(9)$ \\
\hline$C(3)-\operatorname{Zr}(1)-C(2)$ & $32.7(3)$ & $C(9)-\operatorname{Zr}(1)-C(6)$ & $56.5(3)$ \\
\hline $\mathrm{C}(9)-\operatorname{Zr}(1)-\mathrm{C}(2)$ & $148.8(3)$ & $C(2)-\operatorname{Zr}(1)-C(6)$ & $152.7(3)$ \\
\hline$C(20)-Z r(1)-C(5)$ & $130.3(3)$ & $C(5)-\operatorname{Zr}(1)-C(6)$ & $114.5(3)$ \\
\hline$C(19)-\operatorname{Zr}(1)-C(5)$ & $100.7(3)$ & $C(4)-\operatorname{Zr}(1)-C(6)$ & $133.5(3)$ \\
\hline$C(10)-\operatorname{Zr}(1)-C(5)$ & $147.3(3)$ & $C(7)-\operatorname{Zr}(1)-C(6)$ & $34.0(3)$ \\
\hline$C(3)-\operatorname{Zr}(1)-C(5)$ & $54.5(3)$ & $C(8)-\operatorname{Zr}(1)-C(6)$ & $55.3(3)$ \\
\hline$C(9)-\operatorname{Zr}(1)-C(5)$ & $141.8(3)$ & $C(20)-\operatorname{Zr}(1)-C(1)$ & $109.0(3)$ \\
\hline$C(2)-\operatorname{Zr}(1)-C(5)$ & $54.9(2)$ & $C(19)-\operatorname{Zr}(1)-C(1)$ & $71.2(3)$ \\
\hline$C(20)-Z r(1)-C(4)$ & 107.1(3) & $C(10)-\operatorname{Zr}(1)-C(1)$ & $148.9(3)$ \\
\hline$C(19)-\operatorname{Zr}(1)-C(4)$ & $126.1(3)$ & $C(3)-\operatorname{Zr}(1)-C(1)$ & $55.1(3)$ \\
\hline$C(10)-Z r(1)-C(4)$ & $150.7(3)$ & $C(9)-\operatorname{Zr}(1)-C(1)$ & $176.09(8)$ \\
\hline$C(3)-\operatorname{Zr}(1)-C(4)$ & $32.0(3)$ & $C(2)-\operatorname{Zr}(1)-C(1)$ & $33.1(3)$ \\
\hline $\mathrm{C}(9)-\operatorname{Zr}(1)-\mathrm{C}(4)$ & $122.1(3)$ & $C(5)-\operatorname{Zr}(1)-C(1)$ & $34.3(2)$ \\
\hline$C(2)-\operatorname{Zr}(1)-C(4)$ & $53.6(3)$ & $C(4)-\operatorname{Zr}(1)-C(1)$ & $55.1(3)$ \\
\hline$C(5)-\operatorname{Zr}(1)-C(4)$ & $32.8(3)$ & $C(7)-\operatorname{Zr}(1)-C(1)$ & $122.9(3)$ \\
\hline$C(20)-\operatorname{Zr}(1)-C(7)$ & 128.1(3) & $C(8)-\operatorname{Zr}(1)-C(1)$ & $145.6(2)$ \\
\hline$C(19)-\operatorname{Zr}(1)-C(7)$ & $110.0(3)$ & $C(6)-\operatorname{Zr}(1)-C(1)$ & $122.7(3)$ \\
\hline$C(10)-Z r(1)-C(7)$ & $53.1(3)$ & $C(1)-S i(1)-C(22)$ & $115.3(5)$ \\
\hline $\mathrm{C}(3)-\mathrm{Zr}(1)-\mathrm{C}(7)$ & $132.4(3)$ & $C(1)-S i(1)-C(21)$ & 108.1(4) \\
\hline$C(9)-\operatorname{Zr}(1)-C(7)$ & $54.0(3)$ & $C(22)-S i(1)-C(21)$ & $109.6(5)$ \\
\hline$C(2)-\operatorname{Zr}(1)-C(7)$ & $151.6(3)$ & $C(1)-S i(1)-C(23)$ & $107.6(4)$ \\
\hline$C(5)-\operatorname{Zr}(1)-C(7)$ & $96.7(3)$ & $C(22)-S i(1)-C(23)$ & $107.9(5)$ \\
\hline$C(4)-\operatorname{Zr}(1)-C(7)$ & $102.85(9)$ & $C(21)-S i(1)-C(23)$ & $108.0(4)$ \\
\hline$C(20)-\operatorname{Zr}(1)-C(8)$ & $100.2(3)$ & $C(28)-\operatorname{Si}(2)-C(27)$ & $109.8(5)$ \\
\hline $\mathrm{C}(19)-\operatorname{Zr}(1)-\mathrm{C}(8)$ & $130.8(3)$ & $C(28)-S i(2)-C(29)$ & $110.2(5)$ \\
\hline$C(10)-\operatorname{Zr}(1)-C(8)$ & $51.4(3)$ & $C(27)-S i(2)-C(29)$ & $109.2(5)$ \\
\hline $\mathrm{C}(3)-\operatorname{Zr}(1)-\mathrm{C}(8)$ & $116.9(3)$ & $\mathrm{C}(28)-\mathrm{Si}(2)-\mathrm{C}(3)$ & $109.5(4)$ \\
\hline $\mathrm{C}(9)-\operatorname{Zr}(1)-\mathrm{C}(8)$ & $30.5(3)$ & $C(27)-S i(2)-C(3)$ & $108.5(4)$ \\
\hline
\end{tabular}




\begin{tabular}{|c|c|c|c|}
\hline$C(29)-\operatorname{Si}(2)-C(3)$ & $109.6(4)$ & $C(4)-C(5)-C(1)$ & $107.4(7)$ \\
\hline $\mathrm{C}(34)-\mathrm{Si}(3)-\mathrm{C}(35)$ & $109.0(5)$ & $C(14)-C(5)-Z r(1)$ & $122.3(7)$ \\
\hline $\mathrm{C}(34)-\mathrm{Si}(3)-\mathrm{C}(33)$ & $109.0(5)$ & $C(4)-C(5)-Z r(1)$ & $73.8(5)$ \\
\hline $\mathrm{C}(35)-\mathrm{Si}(3)-\mathrm{C}(33)$ & $109.9(5)$ & $C(1)-C(5)-Z r(1)$ & $74.6(4)$ \\
\hline$C(34)-\operatorname{Si}(3)-C(9)$ & $107.0(4)$ & $C(10)-C(6)-C(7)$ & $99.2(8)$ \\
\hline $\mathrm{C}(35)-\mathrm{Si}(3)-\mathrm{C}(9)$ & $107.6(5)$ & $C(10)-C(6)-S i(4)$ & $126.5(7)$ \\
\hline$C(33)-\operatorname{Si}(3)-C(9)$ & $114.3(5)$ & $C(7)-C(6)-S i(4)$ & $132.0(7)$ \\
\hline $\mathrm{C}(6)-\mathrm{Si}(4)-\mathrm{C}(39)$ & $110.3(4)$ & $C(10)-C(6)-Z r(1)$ & $68.9(5)$ \\
\hline$C(6)-\operatorname{Si}(4)-C(40)$ & $110.7(4)$ & $C(7)-C(6)-Z r(1)$ & $72.8(5)$ \\
\hline $\mathrm{C}(39)-\mathrm{Si}(4)-\mathrm{C}(40)$ & $110.8(5)$ & $\operatorname{Si}(4)-C(6)-\operatorname{Zr}(1)$ & $132.6(5)$ \\
\hline$C(6)-\operatorname{Si}(4)-C(41)$ & $110.8(4)$ & $\mathrm{C}(15)-\mathrm{C}(7)-\mathrm{C}(8)$ & $124.5(8)$ \\
\hline $\mathrm{C}(39)-\mathrm{Si}(4)-\mathrm{C}(41)$ & $104.6(5)$ & $\mathrm{C}(15)-\mathrm{C}(7)-\mathrm{C}(6)$ & $127.2(9)$ \\
\hline $\mathrm{C}(40)-\mathrm{Si}(4)-\mathrm{C}(41)$ & $109.5(4)$ & $C(8)-C(7)-C(6)$ & $108.1(7)$ \\
\hline$C(2)-C(1)-C(5)$ & $103.7(7)$ & $C(15)-C(7)-Z r(1)$ & $122.2(6)$ \\
\hline$C(2)-C(1)-S i(1)$ & $122.1(6)$ & $\mathrm{C}(8)-\mathrm{C}(7)-\operatorname{Zr}(1)$ & $74.3(5)$ \\
\hline$C(5)-C(1)-S i(1)$ & $129.4(6)$ & $C(6)-C(7)-\operatorname{Zr}(1)$ & $73.2(5)$ \\
\hline$C(2)-C(1)-Z r(1)$ & $71.3(5)$ & $\mathrm{C}(9)-\mathrm{C}(8)-\mathrm{C}(7)$ & $111.2(8)$ \\
\hline$C(5)-C(1)-\operatorname{Zr}(1)$ & 71.1(4) & $\mathrm{C}(9)-\mathrm{C}(8)-\mathrm{C}(18)$ & $135.3(8)$ \\
\hline $\operatorname{Si}(1)-C(1)-\operatorname{Zr}(1)$ & $140.3(5)$ & $\mathrm{C}(7)-\mathrm{C}(8)-\mathrm{C}(18)$ & $113.5(7)$ \\
\hline$C(3)-C(2)-C(1)$ & $110.2(7)$ & $\mathrm{C}(9)-\mathrm{C}(8)-\mathrm{Zr}(1)$ & $72.6(5)$ \\
\hline$C(3)-C(2)-\operatorname{Zr}(1)$ & $72.9(5)$ & $\mathrm{C}(7)-\mathrm{C}(8)-\operatorname{Zr}(1)$ & $72.9(5)$ \\
\hline $\mathrm{C}(1)-\mathrm{C}(2)-\operatorname{Zr}(1)$ & $75.6(5)$ & $C(18)-C(8)-\operatorname{Zr}(1)$ & $120.4(6)$ \\
\hline $\mathrm{C}(4)-\mathrm{C}(3)-\mathrm{C}(2)$ & $108.9(7)$ & $\mathrm{C}(8)-\mathrm{C}(9)-\mathrm{C}(10)$ & $105.4(8)$ \\
\hline $\mathrm{C}(4)-\mathrm{C}(3)-\mathrm{Si}(2)$ & $126.3(6)$ & $\mathrm{C}(8)-\mathrm{C}(9)-\mathrm{Si}(3)$ & $125.7(7)$ \\
\hline$C(2)-C(3)-S i(2)$ & $122.0(6)$ & $C(10)-C(9)-S i(3)$ & $122.2(7)$ \\
\hline$C(4)-C(3)-\operatorname{Zr}(1)$ & $75.2(5)$ & $\mathrm{C}(8)-\mathrm{C}(9)-\operatorname{Zr}(1)$ & $76.8(5)$ \\
\hline $\mathrm{C}(2)-\mathrm{C}(3)-\operatorname{Zr}(1)$ & $74.4(5)$ & $C(10)-C(9)-Z r(1)$ & $70.6(5)$ \\
\hline $\operatorname{Si}(2)-C(3)-\operatorname{Zr}(1)$ & $131.6(4)$ & $\operatorname{Si}(3)-C(9)-\operatorname{Zr}(1)$ & $140.1(5)$ \\
\hline$C(3)-C(4)-C(5)$ & $109.7(7)$ & $C(9)-C(10)-C(6)$ & $116.0(8)$ \\
\hline$C(3)-C(4)-C(11)$ & $136.6(8)$ & $\mathrm{C}(9)-\mathrm{C}(10)-\mathrm{Zr}(1)$ & $76.8(6)$ \\
\hline$C(5)-C(4)-C(11)$ & $113.6(8)$ & $C(6)-C(10)-Z r(1)$ & $77.7(5)$ \\
\hline $\mathrm{C}(3)-\mathrm{C}(4)-\operatorname{Zr}(1)$ & $72.8(5)$ & $\mathrm{C}(12)-\mathrm{C}(11)-\mathrm{C}(4)$ & $122.7(9)$ \\
\hline$C(5)-C(4)-Z r(1)$ & $73.4(4)$ & $\mathrm{C}(11)-\mathrm{C}(12)-\mathrm{C}(13)$ & $122.3(9)$ \\
\hline$C(11)-C(4)-\operatorname{Zr}(1)$ & $122.3(6)$ & $\mathrm{C}(12)-\mathrm{C}(13)-\mathrm{C}(14)$ & $122.1(9)$ \\
\hline$C(14)-C(5)-C(4)$ & $125.4(7)$ & $C(5)-C(14)-C(13)$ & $113.3(8)$ \\
\hline $\mathrm{C}(14)-\mathrm{C}(5)-\mathrm{C}(1)$ & $126.9(8)$ & $\mathrm{C}(16)-\mathrm{C}(15)-\mathrm{C}(7)$ & $115.3(9)$ \\
\hline
\end{tabular}




$\begin{array}{ll}\mathrm{C}(15)-\mathrm{C}(16)-\mathrm{C}(17) & 120.9(8) \\ \mathrm{C}(18)-\mathrm{C}(17)-\mathrm{C}(16) & 121.5(9) \\ \mathrm{C}(17)-\mathrm{C}(18)-\mathrm{C}(8) & 123.9(8) \\ \mathrm{O}(1)-\mathrm{C}(19)-\mathrm{Zr}(1) & 175.4(8) \\ \mathrm{O}(2)-\mathrm{C}(20)-\mathrm{Zr}(1) & 176.9(9) \\ \mathrm{C}(24)-\mathrm{C}(23)-\mathrm{C}(26) & 109.3(8) \\ \mathrm{C}(24)-\mathrm{C}(23)-\mathrm{C}(25) & 110.7(9) \\ \mathrm{C}(26)-\mathrm{C}(23)-\mathrm{C}(25) & 109.2(9) \\ \mathrm{C}(24)-\mathrm{C}(23)-\mathrm{Si}(1) & 107.9(7) \\ \mathrm{C}(26)-\mathrm{C}(23)-\mathrm{Si}(1) & 112.7(8) \\ \mathrm{C}(25)-\mathrm{C}(23)-\mathrm{Si}(1) & 107.1(6) \\ \mathrm{C}(32)-\mathrm{C}(29)-\mathrm{C}(30) & 112.2(9) \\ \mathrm{C}(32)-\mathrm{C}(29)-\mathrm{C}(31) & 107.7(9) \\ \mathrm{C}(30)-\mathrm{C}(29)-\mathrm{C}(31) & 108.8(8) \\ \mathrm{C}(32)-\mathrm{C}(29)-\mathrm{Si}(2) & 111.2(7) \\ \mathrm{C}(30)-\mathrm{C}(29)-\mathrm{Si}(2) & 109.7(7) \\ \mathrm{C}(31)-\mathrm{C}(29)-\mathrm{Si}(2) & 107.0(7) \\ \mathrm{C}(38)-\mathrm{C}(35)-\mathrm{C}(36) & 107.6(9) \\ \mathrm{C}(38)-\mathrm{C}(35)-\mathrm{C}(37) & 105.9(9) \\ \mathrm{C}(36)-\mathrm{C}(35)-\mathrm{C}(37) & 106.6(8) \\ \mathrm{C}(38)-\mathrm{C}(35)-\mathrm{Si}(3) & 111.7(7) \\ \mathrm{C}(36)-\mathrm{C}(35)-\mathrm{Si}(3) & 113.2(7) \\ \mathrm{C}(37)-\mathrm{C}(35)-\mathrm{Si}(3) & 111.3(7) \\ \mathrm{C}(44)-\mathrm{C}(41)-\mathrm{C}(43) & 107.4(9) \\ \mathrm{C}(44)-\mathrm{C}(41)-\mathrm{C}(42) & 106.7(9) \\ \mathrm{C}(43)-\mathrm{C}(41)-\mathrm{C}(42) & 105.8(8) \\ \mathrm{C}(44)-\mathrm{C}(41)-\mathrm{Si}(4) & 111.0(7) \\ \mathrm{C}(43)-\mathrm{C}(41)-\mathrm{Si}(4) & 112.1(7) \\ \mathrm{C}(42)-\mathrm{C}(41)-\mathrm{Si}(4) & 113.4(7) \\ & \end{array}$




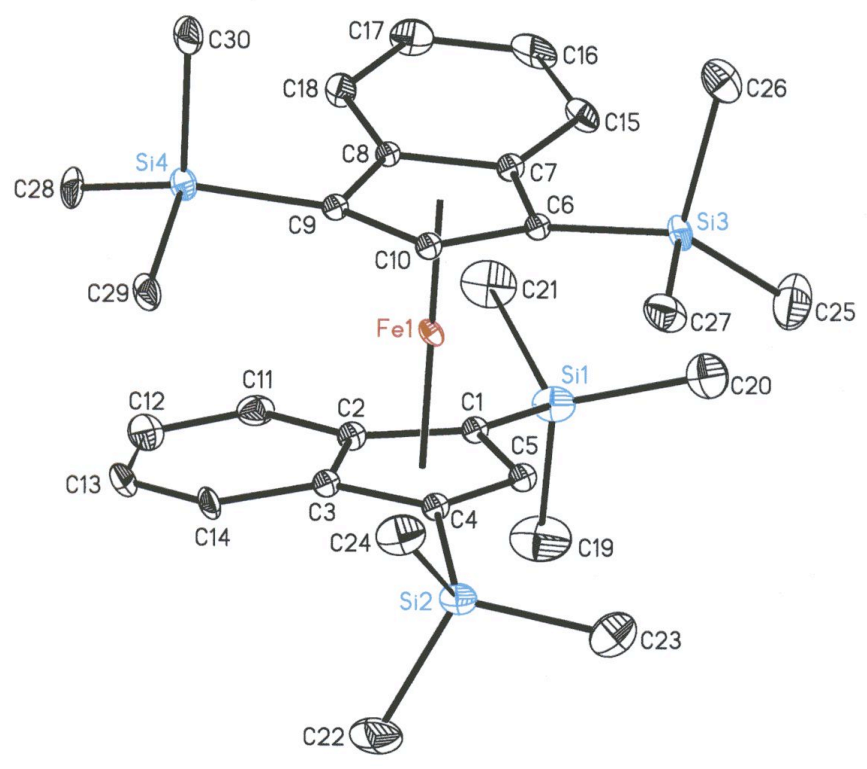

Figure S8. Fully labeled view of $\mathbf{1 3}$ with $30 \%$ probability ellipsoids. Hydrogen atoms omitted for clarity. 
Table S11. Bond lengths $[\AA]$ and angles $\left[^{\circ}\right]$ for

13.

\begin{tabular}{|c|c|}
\hline $\mathrm{Fe}(1)-\mathrm{C}(10)$ & $2.038(4)$ \\
\hline $\mathrm{Fe}(1)-\mathrm{C}(5)$ & $2.045(5)$ \\
\hline $\mathrm{Fe}(1)-\mathrm{C}(4)$ & $2.073(5)$ \\
\hline $\mathrm{Fe}(1)-\mathrm{C}(9)$ & $2.079(5)$ \\
\hline $\mathrm{Fe}(1)-\mathrm{C}(1)$ & $2.080(4)$ \\
\hline $\mathrm{Fe}(1)-\mathrm{C}(6)$ & $2.081(4)$ \\
\hline $\mathrm{Fe}(1)-\mathrm{C}(2)$ & $2.119(4)$ \\
\hline $\mathrm{Fe}(1)-\mathrm{C}(7)$ & $2.123(5)$ \\
\hline $\mathrm{Fe}(1)-\mathrm{C}(3)$ & $2.131(5)$ \\
\hline $\mathrm{Fe}(1)-\mathrm{C}(8)$ & $2.141(5)$ \\
\hline Si(1)-C(19) & $1.860(6)$ \\
\hline Si(1)-C(21) & $1.864(6)$ \\
\hline Si(1)-C(20) & $1.865(6)$ \\
\hline $\operatorname{Si}(1)-C(1)$ & $1.867(5)$ \\
\hline $\operatorname{Si}(2)-C(23)$ & $1.854(6)$ \\
\hline $\operatorname{Si}(2)-C(22)$ & $1.870(6)$ \\
\hline $\operatorname{Si}(2)-C(24)$ & $1.863(5)$ \\
\hline $\operatorname{Si}(2)-C(4)$ & $1.859(5)$ \\
\hline $\mathrm{Si}(3)-\mathrm{C}(25)$ & $1.859(6)$ \\
\hline $\mathrm{Si}(3)-\mathrm{C}(6)$ & $1.861(5)$ \\
\hline Si(3)-C(26) & $1.860(5)$ \\
\hline Si(3)-C(27) & $1.877(6)$ \\
\hline Si(4)-C(29) & $1.862(5)$ \\
\hline $\operatorname{Si}(4)-C(9)$ & $1.866(5)$ \\
\hline $\operatorname{Si}(4)-C(30)$ & $1.870(5)$ \\
\hline $\mathrm{Si}(4)-\mathrm{C}(28)$ & $1.869(5)$ \\
\hline$C(1)-C(5)$ & $1.425(6)$ \\
\hline$C(1)-C(2)$ & $1.438(7)$ \\
\hline $\mathrm{C}(2)-\mathrm{C}(11)$ & $1.435(7)$ \\
\hline $\mathrm{C}(2)-\mathrm{C}(3)$ & $1.444(7)$ \\
\hline$C(3)-C(14)$ & $1.419(7)$ \\
\hline$C(3)-C(4)$ & $1.451(7)$ \\
\hline
\end{tabular}

\begin{tabular}{|c|c|}
\hline$C(4)-C(5)$ & $1.432(7)$ \\
\hline$C(6)-C(10)$ & $1.429(7)$ \\
\hline$C(6)-C(7)$ & $1.442(7)$ \\
\hline$C(7)-C(8)$ & $1.432(7)$ \\
\hline$C(7)-C(15)$ & $1.433(7)$ \\
\hline $\mathrm{C}(8)-\mathrm{C}(18)$ & $1.409(7)$ \\
\hline$C(8)-C(9)$ & $1.453(7)$ \\
\hline $\mathrm{C}(9)-\mathrm{C}(10)$ & $1.412(7)$ \\
\hline $\mathrm{C}(11)-\mathrm{C}(12)$ & $1.345(8)$ \\
\hline$C(12)-C(13)$ & $1.423(8)$ \\
\hline$C(13)-C(14)$ & $1.343(7)$ \\
\hline$C(15)-C(16)$ & $1.343(7)$ \\
\hline$C(16)-C(17)$ & $1.431(8)$ \\
\hline $\mathrm{C}(17)-\mathrm{C}(18)$ & $1.350(7)$ \\
\hline $\mathrm{C}(10)-\mathrm{Fe}(1)-\mathrm{C}(5)$ & $128.19(18)$ \\
\hline $\mathrm{C}(10)-\mathrm{Fe}(1)-\mathrm{C}(4)$ & $106.24(18)$ \\
\hline $\mathrm{C}(5)-\mathrm{Fe}(1)-\mathrm{C}(4)$ & $40.69(19)$ \\
\hline $\mathrm{C}(10)-\mathrm{Fe}(1)-\mathrm{C}(9)$ & $40.10(18)$ \\
\hline $\mathrm{C}(5)-\mathrm{Fe}(1)-\mathrm{C}(9)$ & $164.68(18)$ \\
\hline $\mathrm{C}(4)-\mathrm{Fe}(1)-\mathrm{C}(9)$ & $125.88(19)$ \\
\hline $\mathrm{C}(10)-\mathrm{Fe}(1)-\mathrm{C}(1)$ & $166.10(19)$ \\
\hline $\mathrm{C}(5)-\mathrm{Fe}(1)-\mathrm{C}(1)$ & $40.42(18)$ \\
\hline $\mathrm{C}(4)-\mathrm{Fe}(1)-\mathrm{C}(1)$ & $69.62(18)$ \\
\hline $\mathrm{C}(9)-\mathrm{Fe}(1)-\mathrm{C}(1)$ & $152.88(18)$ \\
\hline $\mathrm{C}(10)-\mathrm{Fe}(1)-\mathrm{C}(6)$ & $40.57(18)$ \\
\hline $\mathrm{C}(5)-\mathrm{Fe}(1)-\mathrm{C}(6)$ & 107.81(18) \\
\hline $\mathrm{C}(4)-\mathrm{Fe}(1)-\mathrm{C}(6)$ & $115.50(18)$ \\
\hline $\mathrm{C}(9)-\mathrm{Fe}(1)-\mathrm{C}(6)$ & $69.17(18)$ \\
\hline $\mathrm{C}(1)-\mathrm{Fe}(1)-\mathrm{C}(6)$ & $128.13(19)$ \\
\hline $\mathrm{C}(10)-\mathrm{Fe}(1)-\mathrm{C}(2)$ & $152.0(2)$ \\
\hline $\mathrm{C}(5)-\mathrm{Fe}(1)-\mathrm{C}(2)$ & $66.11(18)$ \\
\hline $\mathrm{C}(4)-\mathrm{Fe}(1)-\mathrm{C}(2)$ & $67.87(18)$ \\
\hline $\mathrm{C}(9)-\mathrm{Fe}(1)-\mathrm{C}(2)$ & $119.73(18)$ \\
\hline $\mathrm{C}(1)-\mathrm{Fe}(1)-\mathrm{C}(2)$ & $40.04(19)$ \\
\hline
\end{tabular}




\begin{tabular}{|c|c|}
\hline $\mathrm{C}(6)-\mathrm{Fe}(1)-\mathrm{C}(2)$ & $167.31(19)$ \\
\hline $\mathrm{C}(10)-\mathrm{Fe}(1)-\mathrm{C}(7)$ & $66.09(18)$ \\
\hline $\mathrm{C}(5)-\mathrm{Fe}(1)-\mathrm{C}(7)$ & $120.58(19)$ \\
\hline $\mathrm{C}(4)-\mathrm{Fe}(1)-\mathrm{C}(7)$ & $151.03(19)$ \\
\hline $\mathrm{C}(9)-\mathrm{Fe}(1)-\mathrm{C}(7)$ & $67.43(18)$ \\
\hline $\mathrm{C}(1)-\mathrm{Fe}(1)-\mathrm{C}(7)$ & $110.73(19)$ \\
\hline $\mathrm{C}(6)-\mathrm{Fe}(1)-\mathrm{C}(7)$ & $40.09(18)$ \\
\hline $\mathrm{C}(2)-\mathrm{Fe}(1)-\mathrm{C}(7)$ & $132.04(18)$ \\
\hline $\mathrm{C}(10)-\mathrm{Fe}(1)-\mathrm{C}(3)$ & 118.01(19) \\
\hline $\mathrm{C}(5)-\mathrm{Fe}(1)-\mathrm{C}(3)$ & $66.52(19)$ \\
\hline $\mathrm{C}(4)-\mathrm{Fe}(1)-\mathrm{C}(3)$ & $40.35(18)$ \\
\hline C(9)-Fe(1)-C(3) & $108.10(19)$ \\
\hline $\mathrm{C}(1)-\mathrm{Fe}(1)-\mathrm{C}(3)$ & $67.99(18)$ \\
\hline $\mathrm{C}(6)-\mathrm{Fe}(1)-\mathrm{C}(3)$ & $149.93(18)$ \\
\hline $\mathrm{C}(2)-\mathrm{Fe}(1)-\mathrm{C}(3)$ & $39.74(18)$ \\
\hline $\mathrm{C}(7)-\mathrm{Fe}(1)-\mathrm{C}(3)$ & $168.38(18)$ \\
\hline $\mathrm{C}(10)-\mathrm{Fe}(1)-\mathrm{C}(8)$ & $66.07(18)$ \\
\hline $\mathrm{C}(5)-\mathrm{Fe}(1)-\mathrm{C}(8)$ & $153.82(19)$ \\
\hline $\mathrm{C}(4)-\mathrm{Fe}(1)-\mathrm{C}(8)$ & $165.34(19)$ \\
\hline $\mathrm{C}(9)-\mathrm{Fe}(1)-\mathrm{C}(8)$ & $40.24(18)$ \\
\hline $\mathrm{C}(1)-\mathrm{Fe}(1)-\mathrm{C}(8)$ & $120.83(18)$ \\
\hline $\mathrm{C}(6)-\mathrm{Fe}(1)-\mathrm{C}(8)$ & $67.54(17)$ \\
\hline $\mathrm{C}(2)-\mathrm{Fe}(1)-\mathrm{C}(8)$ & $112.56(18)$ \\
\hline $\mathrm{C}(7)-\mathrm{Fe}(1)-\mathrm{C}(8)$ & $39.25(18)$ \\
\hline $\mathrm{C}(3)-\mathrm{Fe}(1)-\mathrm{C}(8)$ & $130.61(18)$ \\
\hline $\mathrm{C}(19)-\mathrm{Si}(1)-\mathrm{C}(21)$ & $108.0(3)$ \\
\hline $\mathrm{C}(19)-\mathrm{Si}(1)-\mathrm{C}(20)$ & $109.5(3)$ \\
\hline$C(21)-\operatorname{Si}(1)-C(20)$ & $111.6(3)$ \\
\hline C(19)-Si(1)-C(1) & 107.2(3) \\
\hline $\mathrm{C}(21)-\mathrm{Si}(1)-\mathrm{C}(1)$ & $111.5(2)$ \\
\hline$C(20)-\operatorname{Si}(1)-C(1)$ & $108.9(2)$ \\
\hline$C(23)-\operatorname{Si}(2)-C(22)$ & $108.4(3)$ \\
\hline $\mathrm{C}(23)-\mathrm{Si}(2)-\mathrm{C}(24)$ & $111.8(3)$ \\
\hline $\mathrm{C}(22)-\mathrm{Si}(2)-\mathrm{C}(24)$ & 107.2(3) \\
\hline $\mathrm{C}(23)-\mathrm{Si}(2)-\mathrm{C}(4)$ & 109.1(3) \\
\hline$C(22)-\operatorname{Si}(2)-C(4)$ & $106.5(2)$ \\
\hline
\end{tabular}

\begin{tabular}{|c|c|}
\hline$C(24)-\operatorname{Si}(2)-C(4)$ & $113.6(2)$ \\
\hline$C(25)-\operatorname{Si}(3)-C(6)$ & $114.0(2)$ \\
\hline$C(25)-\operatorname{Si}(3)-C(26)$ & $108.9(3)$ \\
\hline $\mathrm{C}(6)-\mathrm{Si}(3)-\mathrm{C}(26)$ & $106.2(2)$ \\
\hline $\mathrm{C}(25)-\mathrm{Si}(3)-\mathrm{C}(27)$ & $109.6(3)$ \\
\hline$C(6)-\operatorname{Si}(3)-C(27)$ & $108.8(3)$ \\
\hline $\mathrm{C}(26)-\mathrm{Si}(3)-\mathrm{C}(27)$ & $109.3(2)$ \\
\hline $\mathrm{C}(29)-\mathrm{Si}(4)-\mathrm{C}(9)$ & $109.9(2)$ \\
\hline $\mathrm{C}(29)-\mathrm{Si}(4)-\mathrm{C}(30)$ & $110.8(2)$ \\
\hline$C(9)-S i(4)-C(30)$ & $103.0(2)$ \\
\hline $\mathrm{C}(29)-\mathrm{Si}(4)-\mathrm{C}(28)$ & $110.5(2)$ \\
\hline $\mathrm{C}(9)-\mathrm{Si}(4)-\mathrm{C}(28)$ & $114.0(2)$ \\
\hline $\mathrm{C}(30)-\mathrm{Si}(4)-\mathrm{C}(28)$ & $108.4(2)$ \\
\hline$C(5)-C(1)-C(2)$ & $105.0(4)$ \\
\hline$C(5)-C(1)-S i(1)$ & $126.0(4)$ \\
\hline$C(2)-C(1)-S i(1)$ & $127.1(3)$ \\
\hline $\mathrm{C}(5)-\mathrm{C}(1)-\mathrm{Fe}(1)$ & $68.5(2)$ \\
\hline $\mathrm{C}(2)-\mathrm{C}(1)-\mathrm{Fe}(1)$ & $71.4(2)$ \\
\hline $\operatorname{Si}(1)-\mathrm{C}(1)-\mathrm{Fe}(1)$ & $136.3(3)$ \\
\hline $\mathrm{C}(11)-\mathrm{C}(2)-\mathrm{C}(3)$ & $117.7(5)$ \\
\hline $\mathrm{C}(11)-\mathrm{C}(2)-\mathrm{C}(1)$ & $132.7(5)$ \\
\hline $\mathrm{C}(3)-\mathrm{C}(2)-\mathrm{C}(1)$ & $109.5(4)$ \\
\hline $\mathrm{C}(11)-\mathrm{C}(2)-\mathrm{Fe}(1)$ & $129.7(3)$ \\
\hline $\mathrm{C}(3)-\mathrm{C}(2)-\mathrm{Fe}(1)$ & $70.6(2)$ \\
\hline $\mathrm{C}(1)-\mathrm{C}(2)-\mathrm{Fe}(1)$ & $68.5(2)$ \\
\hline $\mathrm{C}(14)-\mathrm{C}(3)-\mathrm{C}(2)$ & $119.9(4)$ \\
\hline$C(14)-C(3)-C(4)$ & $132.2(4)$ \\
\hline$C(2)-C(3)-C(4)$ & $107.9(4)$ \\
\hline $\mathrm{C}(14)-\mathrm{C}(3)-\mathrm{Fe}(1)$ & $130.3(3)$ \\
\hline $\mathrm{C}(2)-\mathrm{C}(3)-\mathrm{Fe}(1)$ & $69.7(3)$ \\
\hline $\mathrm{C}(4)-\mathrm{C}(3)-\mathrm{Fe}(1)$ & $67.7(3)$ \\
\hline$C(5)-C(4)-C(3)$ & $105.3(4)$ \\
\hline $\mathrm{C}(5)-\mathrm{C}(4)-\mathrm{Si}(2)$ & $125.2(4)$ \\
\hline$C(3)-C(4)-S i(2)$ & $126.7(4)$ \\
\hline $\mathrm{C}(5)-\mathrm{C}(4)-\mathrm{Fe}(1)$ & $68.6(3)$ \\
\hline $\mathrm{C}(3)-\mathrm{C}(4)-\mathrm{Fe}(1)$ & $72.0(3)$ \\
\hline
\end{tabular}




$\begin{array}{lrlr}\mathrm{Si}(2)-\mathrm{C}(4)-\mathrm{Fe}(1) & 138.5(2) & \mathrm{C}(7)-\mathrm{C}(8)-\mathrm{Fe}(1) & 69.7(3) \\ \mathrm{C}(4)-\mathrm{C}(5)-\mathrm{C}(1) & 112.2(4) & \mathrm{C}(9)-\mathrm{C}(8)-\mathrm{Fe}(1) & 67.6(3) \\ \mathrm{C}(4)-\mathrm{C}(5)-\mathrm{Fe}(1) & 70.7(3) & \mathrm{C}(10)-\mathrm{C}(9)-\mathrm{C}(8) & 105.4(4) \\ \mathrm{C}(1)-\mathrm{C}(5)-\mathrm{Fe}(1) & 71.1(3) & \mathrm{C}(10)-\mathrm{C}(9)-\mathrm{Si}(4) & 125.7(4) \\ \mathrm{C}(10)-\mathrm{C}(6)-\mathrm{C}(7) & 104.5(4) & \mathrm{C}(8)-\mathrm{C}(9)-\mathrm{Si}(4) & 125.9(4) \\ \mathrm{C}(10)-\mathrm{C}(6)-\mathrm{Si}(3) & 126.2(3) & \mathrm{C}(10)-\mathrm{C}(9)-\mathrm{Fe}(1) & 68.4(3) \\ \mathrm{C}(7)-\mathrm{C}(6)-\mathrm{Si}(3) & 126.5(4) & \mathrm{C}(8)-\mathrm{C}(9)-\mathrm{Fe}(1) & 72.2(3) \\ \mathrm{C}(10)-\mathrm{C}(6)-\mathrm{Fe}(1) & 68.1(2) & \mathrm{Si}(4)-\mathrm{C}(9)-\mathrm{Fe}(1) & 138.6(2) \\ \mathrm{C}(7)-\mathrm{C}(6)-\mathrm{Fe}(1) & 71.5(2) & \mathrm{C}(9)-\mathrm{C}(10)-\mathrm{C}(6) & 112.5(4) \\ \mathrm{Si}(3)-\mathrm{C}(6)-\mathrm{Fe}(1) & 138.6(3) & \mathrm{C}(9)-\mathrm{C}(10)-\mathrm{Fe}(1) & 71.5(2) \\ \mathrm{C}(8)-\mathrm{C}(7)-\mathrm{C}(15) & 118.8(4) & \mathrm{C}(6)-\mathrm{C}(10)-\mathrm{Fe}(1) & 71.4(2) \\ \mathrm{C}(8)-\mathrm{C}(7)-\mathrm{C}(6) & 109.5(4) & \mathrm{C}(12)-\mathrm{C}(11)-\mathrm{C}(2) & 120.5(5) \\ \mathrm{C}(15)-\mathrm{C}(7)-\mathrm{C}(6) & 131.4(5) & \mathrm{C}(11)-\mathrm{C}(12)-\mathrm{C}(13) & 120.7(5) \\ \mathrm{C}(8)-\mathrm{C}(7)-\mathrm{Fe}(1) & 71.0(3) & \mathrm{C}(14)-\mathrm{C}(13)-\mathrm{C}(12) & 121.9(5) \\ \mathrm{C}(15)-\mathrm{C}(7)-\mathrm{Fe}(1) & 131.1(3) & \mathrm{C}(13)-\mathrm{C}(14)-\mathrm{C}(3) & 119.4(5) \\ \mathrm{C}(6)-\mathrm{C}(7)-\mathrm{Fe}(1) & 68.4(3) & \mathrm{C}(16)-\mathrm{C}(15)-\mathrm{C}(7) & 119.0(5) \\ \mathrm{C}(18)-\mathrm{C}(8)-\mathrm{C}(7) & 119.9(4) & \mathrm{C}(15)-\mathrm{C}(16)-\mathrm{C}(17) & 121.9(5) \\ \mathrm{C}(18)-\mathrm{C}(8)-\mathrm{C}(9) & 132.0(5) & \mathrm{C}(18)-\mathrm{C}(17)-\mathrm{C}(16) & 120.4(5) \\ \mathrm{C}(7)-\mathrm{C}(8)-\mathrm{C}(9) & 107.9(4) & \mathrm{C}(17)-\mathrm{C}(18)-\mathrm{C}(8) & 119.8(5) \\ \mathrm{C}(18)-\mathrm{C}(8)-\mathrm{Fe}(1) & 131.8(3) & & \end{array}$




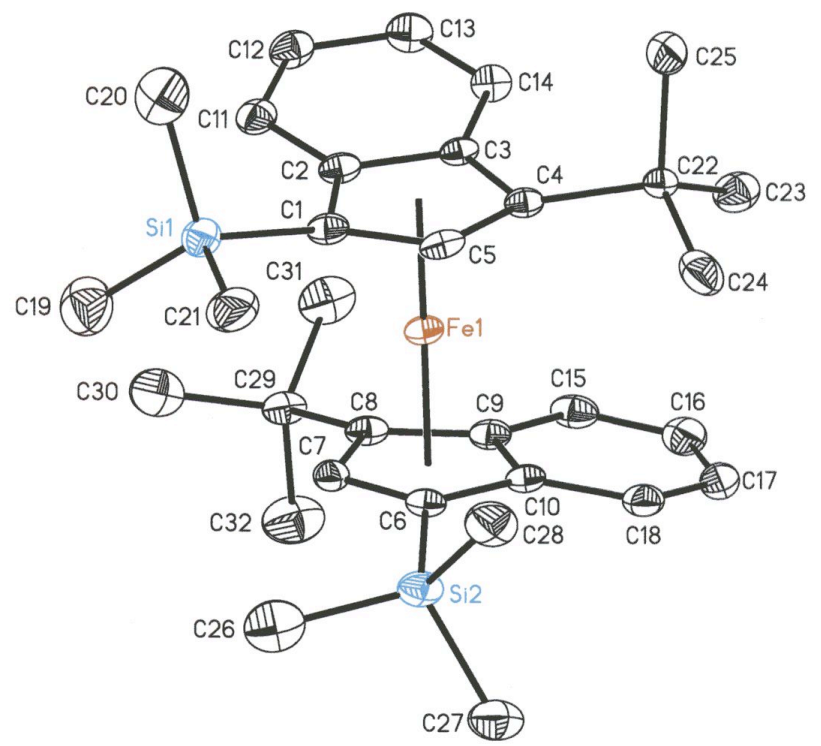

Figure S7. Fully labeled view of the molecular structure of $\mathbf{r a c}-\mathbf{1 7}$ at $30 \%$ probability ellipsoids. Hydrogen atoms omitted for clarity. 


\begin{tabular}{|c|c|c|c|}
\hline Table S12. & Bond lengths $[\AA]$ and angles $\left[{ }^{\circ}\right]$ for & $C(9)-C(10)$ & $1.435(5)$ \\
\hline rac-17. & & $C(10)-C(18)$ & $1.432(5)$ \\
\hline & & $C(11)-C(12)$ & $1.354(5)$ \\
\hline $\mathrm{Fe}(1)-\mathrm{C}(5)$ & $2.048(4)$ & $C(12)-C(13)$ & $1.415(5)$ \\
\hline $\mathrm{Fe}(1)-\mathrm{C}(7)$ & $2.053(4)$ & $C(13)-C(14)$ & $1.358(5)$ \\
\hline $\mathrm{Fe}(1)-\mathrm{C}(6)$ & $2.083(3)$ & $C(15)-C(16)$ & $1.358(6)$ \\
\hline $\mathrm{Fe}(1)-\mathrm{C}(4)$ & $2.084(3)$ & $C(16)-C(17)$ & $1.419(6)$ \\
\hline $\mathrm{Fe}(1)-\mathrm{C}(1)$ & $2.090(3)$ & $\mathrm{C}(17)-\mathrm{C}(18)$ & $1.362(5)$ \\
\hline $\mathrm{Fe}(1)-\mathrm{C}(8)$ & $2.092(3)$ & $C(22)-C(24)$ & $1.592(5)$ \\
\hline $\mathrm{Fe}(1)-\mathrm{C}(10)$ & $2.135(3)$ & $C(22)-C(25)$ & $1.626(4)$ \\
\hline $\mathrm{Fe}(1)-\mathrm{C}(9)$ & $2.147(3)$ & $C(22)-C(23)$ & $1.641(4)$ \\
\hline $\mathrm{Fe}(1)-\mathrm{C}(2)$ & $2.148(3)$ & $C(29)-C(30)$ & $1.572(5)$ \\
\hline $\mathrm{Fe}(1)-\mathrm{C}(3)$ & $2.155(3)$ & $C(29)-C(32)$ & $1.600(5)$ \\
\hline $\mathrm{Si}(1)-\mathrm{C}(21)$ & $1.750(6)$ & $C(29)-C(31)$ & $1.594(5)$ \\
\hline $\operatorname{Si}(1)-C(20)$ & $1.762(5)$ & & \\
\hline $\operatorname{Si}(1)-C(19)$ & $1.782(5)$ & $\mathrm{C}(5)-\mathrm{Fe}(1)-\mathrm{C}(7)$ & $127.97(15)$ \\
\hline $\mathrm{Si}(1)-\mathrm{C}(1)$ & $1.791(4)$ & $\mathrm{C}(5)-\mathrm{Fe}(1)-\mathrm{C}(6)$ & $106.10(14)$ \\
\hline $\operatorname{Si}(2)-C(26)$ & $1.792(5)$ & $\mathrm{C}(7)-\mathrm{Fe}(1)-\mathrm{C}(6)$ & $40.34(14)$ \\
\hline $\mathrm{Si}(2)-\mathrm{C}(28)$ & $1.811(4)$ & $\mathrm{C}(5)-\mathrm{Fe}(1)-\mathrm{C}(4)$ & $40.22(14)$ \\
\hline $\operatorname{Si}(2)-C(6)$ & $1.820(3)$ & $\mathrm{C}(7)-\mathrm{Fe}(1)-\mathrm{C}(4)$ & $165.12(14)$ \\
\hline $\operatorname{Si}(2)-C(27)$ & $1.818(4)$ & $\mathrm{C}(6)-\mathrm{Fe}(1)-\mathrm{C}(4)$ & $126.85(13)$ \\
\hline$C(1)-C(5)$ & $1.434(5)$ & $\mathrm{C}(5)-\mathrm{Fe}(1)-\mathrm{C}(1)$ & $40.53(14)$ \\
\hline$C(1)-C(2)$ & $1.444(5)$ & $\mathrm{C}(7)-\mathrm{Fe}(1)-\mathrm{C}(1)$ & $107.33(15)$ \\
\hline$C(2)-C(11)$ & $1.432(5)$ & $\mathrm{C}(6)-\mathrm{Fe}(1)-\mathrm{C}(1)$ & $114.65(13)$ \\
\hline$C(2)-C(3)$ & $1.440(5)$ & $\mathrm{C}(4)-\mathrm{Fe}(1)-\mathrm{C}(1)$ & $69.02(14)$ \\
\hline$C(3)-C(14)$ & $1.422(5)$ & $\mathrm{C}(5)-\mathrm{Fe}(1)-\mathrm{C}(8)$ & $165.74(15)$ \\
\hline$C(3)-C(4)$ & $1.445(5)$ & $\mathrm{C}(7)-\mathrm{Fe}(1)-\mathrm{C}(8)$ & $39.69(14)$ \\
\hline$C(4)-C(5)$ & $1.421(5)$ & $\mathrm{C}(6)-\mathrm{Fe}(1)-\mathrm{C}(8)$ & $68.48(13)$ \\
\hline$C(4)-C(22)$ & $1.666(4)$ & $\mathrm{C}(4)-\mathrm{Fe}(1)-\mathrm{C}(8)$ & $153.40(14)$ \\
\hline$C(6)-C(7)$ & $1.426(5)$ & $\mathrm{C}(1)-\mathrm{Fe}(1)-\mathrm{C}(8)$ & $128.39(14)$ \\
\hline$C(6)-C(10)$ & $1.445(5)$ & $\mathrm{C}(5)-\mathrm{Fe}(1)-\mathrm{C}(10)$ & $118.02(14)$ \\
\hline$C(7)-C(8)$ & $1.407(5)$ & $\mathrm{C}(7)-\mathrm{Fe}(1)-\mathrm{C}(10)$ & $65.91(14)$ \\
\hline $\mathrm{C}(8)-\mathrm{C}(9)$ & $1.449(5)$ & $C(6)-\mathrm{Fe}(1)-\mathrm{C}(10)$ & $40.04(13)$ \\
\hline$C(8)-C(29)$ & $1.617(4)$ & $\mathrm{C}(4)-\mathrm{Fe}(1)-\mathrm{C}(10)$ & $109.33(13)$ \\
\hline$C(9)-C(15)$ & $1.426(5)$ & $\mathrm{C}(1)-\mathrm{Fe}(1)-\mathrm{C}(10)$ & $149.07(13)$ \\
\hline
\end{tabular}




\begin{tabular}{|c|c|c|c|}
\hline $\mathrm{C}(8)-\mathrm{Fe}(1)-\mathrm{C}(10)$ & $66.95(13)$ & $C(6)-S i(2)-C(27)$ & $105.74(19)$ \\
\hline $\mathrm{C}(5)-\mathrm{Fe}(1)-\mathrm{C}(9)$ & $151.62(15)$ & $\mathrm{C}(5)-\mathrm{C}(1)-\mathrm{C}(2)$ & $105.0(3)$ \\
\hline $\mathrm{C}(7)-\mathrm{Fe}(1)-\mathrm{C}(9)$ & $65.79(14)$ & $C(5)-C(1)-S i(1)$ & $125.3(3)$ \\
\hline $\mathrm{C}(6)-\mathrm{Fe}(1)-\mathrm{C}(9)$ & $67.29(13)$ & $C(2)-C(1)-S i(1)$ & $126.4(3)$ \\
\hline $\mathrm{C}(4)-\mathrm{Fe}(1)-\mathrm{C}(9)$ & $120.26(14)$ & $\mathrm{C}(5)-\mathrm{C}(1)-\mathrm{Fe}(1)$ & $68.2(2)$ \\
\hline $\mathrm{C}(1)-\mathrm{Fe}(1)-\mathrm{C}(9)$ & $167.83(14)$ & $\mathrm{C}(2)-\mathrm{C}(1)-\mathrm{Fe}(1)$ & $72.25(19)$ \\
\hline $\mathrm{C}(8)-\mathrm{Fe}(1)-\mathrm{C}(9)$ & $39.95(14)$ & $\mathrm{Si}(1)-\mathrm{C}(1)-\mathrm{Fe}(1)$ & $139.4(2)$ \\
\hline $\mathrm{C}(10)-\mathrm{Fe}(1)-\mathrm{C}(9)$ & $39.17(13)$ & $\mathrm{C}(11)-\mathrm{C}(2)-\mathrm{C}(1)$ & $131.4(3)$ \\
\hline $\mathrm{C}(5)-\mathrm{Fe}(1)-\mathrm{C}(2)$ & $65.90(14)$ & $\mathrm{C}(11)-\mathrm{C}(2)-\mathrm{C}(3)$ & 119.3(3) \\
\hline $\mathrm{C}(7)-\mathrm{Fe}(1)-\mathrm{C}(2)$ & $119.93(14)$ & $\mathrm{C}(1)-\mathrm{C}(2)-\mathrm{C}(3)$ & $108.9(3)$ \\
\hline $\mathrm{C}(6)-\mathrm{Fe}(1)-\mathrm{C}(2)$ & $149.69(14)$ & $\mathrm{C}(11)-\mathrm{C}(2)-\mathrm{Fe}(1)$ & $132.5(2)$ \\
\hline $\mathrm{C}(4)-\mathrm{Fe}(1)-\mathrm{C}(2)$ & $67.14(13)$ & $\mathrm{C}(1)-\mathrm{C}(2)-\mathrm{Fe}(1)$ & $67.94(19)$ \\
\hline $\mathrm{C}(1)-\mathrm{Fe}(1)-\mathrm{C}(2)$ & $39.82(13)$ & $\mathrm{C}(3)-\mathrm{C}(2)-\mathrm{Fe}(1)$ & 70.71(19) \\
\hline $\mathrm{C}(8)-\mathrm{Fe}(1)-\mathrm{C}(2)$ & $111.67(13)$ & $\mathrm{C}(14)-\mathrm{C}(3)-\mathrm{C}(4)$ & $132.4(3)$ \\
\hline $\mathrm{C}(10)-\mathrm{Fe}(1)-\mathrm{C}(2)$ & $169.97(13)$ & $\mathrm{C}(14)-\mathrm{C}(3)-\mathrm{C}(2)$ & $118.9(3)$ \\
\hline $\mathrm{C}(9)-\mathrm{Fe}(1)-\mathrm{C}(2)$ & $133.50(13)$ & $\mathrm{C}(4)-\mathrm{C}(3)-\mathrm{C}(2)$ & $108.4(3)$ \\
\hline $\mathrm{C}(5)-\mathrm{Fe}(1)-\mathrm{C}(3)$ & $65.77(13)$ & $\mathrm{C}(14)-\mathrm{C}(3)-\mathrm{Fe}(1)$ & $133.0(2)$ \\
\hline $\mathrm{C}(7)-\mathrm{Fe}(1)-\mathrm{C}(3)$ & $153.39(14)$ & $\mathrm{C}(4)-\mathrm{C}(3)-\mathrm{Fe}(1)$ & $67.45(18)$ \\
\hline $\mathrm{C}(6)-\mathrm{Fe}(1)-\mathrm{C}(3)$ & $166.25(13)$ & $\mathrm{C}(2)-\mathrm{C}(3)-\mathrm{Fe}(1)$ & $70.18(18)$ \\
\hline $\mathrm{C}(4)-\mathrm{Fe}(1)-\mathrm{C}(3)$ & $39.83(13)$ & $\mathrm{C}(5)-\mathrm{C}(4)-\mathrm{C}(3)$ & 105.6(3) \\
\hline $\mathrm{C}(1)-\mathrm{Fe}(1)-\mathrm{C}(3)$ & $67.12(13)$ & $\mathrm{C}(5)-\mathrm{C}(4)-\mathrm{C}(22)$ & $124.4(3)$ \\
\hline $\mathrm{C}(8)-\mathrm{Fe}(1)-\mathrm{C}(3)$ & $122.05(13)$ & $\mathrm{C}(3)-\mathrm{C}(4)-\mathrm{C}(22)$ & $127.5(3)$ \\
\hline $\mathrm{C}(10)-\mathrm{Fe}(1)-\mathrm{C}(3)$ & $132.34(13)$ & $\mathrm{C}(5)-\mathrm{C}(4)-\mathrm{Fe}(1)$ & $68.5(2)$ \\
\hline $\mathrm{C}(9)-\mathrm{Fe}(1)-\mathrm{C}(3)$ & $114.12(13)$ & $\mathrm{C}(3)-\mathrm{C}(4)-\mathrm{Fe}(1)$ & $72.73(19)$ \\
\hline $\mathrm{C}(2)-\mathrm{Fe}(1)-\mathrm{C}(3)$ & $39.12(13)$ & $\mathrm{C}(22)-\mathrm{C}(4)-\mathrm{Fe}(1)$ & $136.8(2)$ \\
\hline $\mathrm{C}(21)-\mathrm{Si}(1)-\mathrm{C}(20)$ & $110.1(2)$ & $C(4)-C(5)-C(1)$ & $111.9(3)$ \\
\hline $\mathrm{C}(21)-\mathrm{Si}(1)-\mathrm{C}(19)$ & $110.5(3)$ & $\mathrm{C}(4)-\mathrm{C}(5)-\mathrm{Fe}(1)$ & $71.3(2)$ \\
\hline $\mathrm{C}(20)-\mathrm{Si}(1)-\mathrm{C}(19)$ & 109.1(3) & $\mathrm{C}(1)-\mathrm{C}(5)-\mathrm{Fe}(1)$ & $71.3(2)$ \\
\hline $\mathrm{C}(21)-\mathrm{Si}(1)-\mathrm{C}(1)$ & $109.5(2)$ & $C(7)-C(6)-C(10)$ & $105.1(3)$ \\
\hline$C(20)-\operatorname{Si}(1)-C(1)$ & $105.70(19)$ & $C(7)-C(6)-S i(2)$ & $125.8(3)$ \\
\hline C(19)-Si(1)-C(1) & $111.7(2)$ & $\mathrm{C}(10)-\mathrm{C}(6)-\mathrm{Si}(2)$ & $126.6(3)$ \\
\hline $\mathrm{C}(26)-\mathrm{Si}(2)-\mathrm{C}(28)$ & $112.6(2)$ & $\mathrm{C}(7)-\mathrm{C}(6)-\mathrm{Fe}(1)$ & $68.69(19)$ \\
\hline $\mathrm{C}(26)-\mathrm{Si}(2)-\mathrm{C}(6)$ & 109.49(19) & $\mathrm{C}(10)-\mathrm{C}(6)-\mathrm{Fe}(1)$ & 71.91(18) \\
\hline $\mathrm{C}(28)-\mathrm{Si}(2)-\mathrm{C}(6)$ & $113.26(18)$ & $\mathrm{Si}(2)-\mathrm{C}(6)-\mathrm{Fe}(1)$ & 137.33(19) \\
\hline $\mathrm{C}(26)-\mathrm{Si}(2)-\mathrm{C}(27)$ & $107.6(2)$ & $\mathrm{C}(8)-\mathrm{C}(7)-\mathrm{C}(6)$ & $112.0(3)$ \\
\hline $\mathrm{C}(28)-\mathrm{Si}(2)-\mathrm{C}(27)$ & $107.7(2)$ & $\mathrm{C}(8)-\mathrm{C}(7)-\mathrm{Fe}(1)$ & $71.6(2)$ \\
\hline
\end{tabular}




$\begin{array}{lcll}\mathrm{C}(6)-\mathrm{C}(7)-\mathrm{Fe}(1) & 71.0(2) & \mathrm{C}(11)-\mathrm{C}(12)-\mathrm{C}(13) & 120.9(4) \\ \mathrm{C}(7)-\mathrm{C}(8)-\mathrm{C}(9) & 106.1(3) & \mathrm{C}(14)-\mathrm{C}(13)-\mathrm{C}(12) & 121.9(3) \\ \mathrm{C}(7)-\mathrm{C}(8)-\mathrm{C}(29) & 125.3(3) & \mathrm{C}(13)-\mathrm{C}(14)-\mathrm{C}(3) & 119.4(3) \\ \mathrm{C}(9)-\mathrm{C}(8)-\mathrm{C}(29) & 127.2(3) & \mathrm{C}(16)-\mathrm{C}(15)-\mathrm{C}(9) & 119.8(4) \\ \mathrm{C}(7)-\mathrm{C}(8)-\mathrm{Fe}(1) & 68.7(2) & \mathrm{C}(15)-\mathrm{C}(16)-\mathrm{C}(17) & 121.7(4) \\ \mathrm{C}(9)-\mathrm{C}(8)-\mathrm{Fe}(1) & 72.1(2) & \mathrm{C}(18)-\mathrm{C}(17)-\mathrm{C}(16) & 120.5(4) \\ \mathrm{C}(29)-\mathrm{C}(8)-\mathrm{Fe}(1) & 134.2(2) & \mathrm{C}(17)-\mathrm{C}(18)-\mathrm{C}(10) & 119.7(4) \\ \mathrm{C}(15)-\mathrm{C}(9)-\mathrm{C}(8) & 133.3(3) & \mathrm{C}(24)-\mathrm{C}(22)-\mathrm{C}(25) & 111.7(2) \\ \mathrm{C}(15)-\mathrm{C}(9)-\mathrm{C}(10) & 118.7(3) & \mathrm{C}(24)-\mathrm{C}(22)-\mathrm{C}(23) & 110.9(2) \\ \mathrm{C}(8)-\mathrm{C}(9)-\mathrm{C}(10) & 107.8(3) & \mathrm{C}(25)-\mathrm{C}(22)-\mathrm{C}(23) & 109.1(2) \\ \mathrm{C}(15)-\mathrm{C}(9)-\mathrm{Fe}(1) & 131.1(2) & \mathrm{C}(24)-\mathrm{C}(22)-\mathrm{C}(4) & 110.3(2) \\ \mathrm{C}(8)-\mathrm{C}(9)-\mathrm{Fe}(1) & 67.95(19) & \mathrm{C}(25)-\mathrm{C}(22)-\mathrm{C}(4) & 104.2(2) \\ \mathrm{C}(10)-\mathrm{C}(9)-\mathrm{Fe}(1) & 69.94(19) & \mathrm{C}(23)-\mathrm{C}(22)-\mathrm{C}(4) & 110.4(2) \\ \mathrm{C}(18)-\mathrm{C}(10)-\mathrm{C}(6) & 131.3(3) & \mathrm{C}(30)-\mathrm{C}(29)-\mathrm{C}(32) & 109.5(3) \\ \mathrm{C}(18)-\mathrm{C}(10)-\mathrm{C}(9) & 119.4(3) & \mathrm{C}(30)-\mathrm{C}(29)-\mathrm{C}(31) & 111.4(3) \\ \mathrm{C}(6)-\mathrm{C}(10)-\mathrm{C}(9) & 109.0(3) & \mathrm{C}(32)-\mathrm{C}(29)-\mathrm{C}(31) & 109.5(3) \\ \mathrm{C}(18)-\mathrm{C}(10)-\mathrm{Fe}(1) & 131.8(3) & \mathrm{C}(30)-\mathrm{C}(29)-\mathrm{C}(8) & 109.6(2) \\ \mathrm{C}(6)-\mathrm{C}(10)-\mathrm{Fe}(1) & 68.05(18) & \mathrm{C}(32)-\mathrm{C}(29)-\mathrm{C}(8) & 105.8(3) \\ \mathrm{C}(9)-\mathrm{C}(10)-\mathrm{Fe}(1) & 70.89(19) & \mathrm{C}(31)-\mathrm{C}(29)-\mathrm{C}(8) & 110.8(3) \\ \mathrm{C}(12)-\mathrm{C}(11)-\mathrm{C}(2) & 119.5(4) & & \end{array}$


(a)

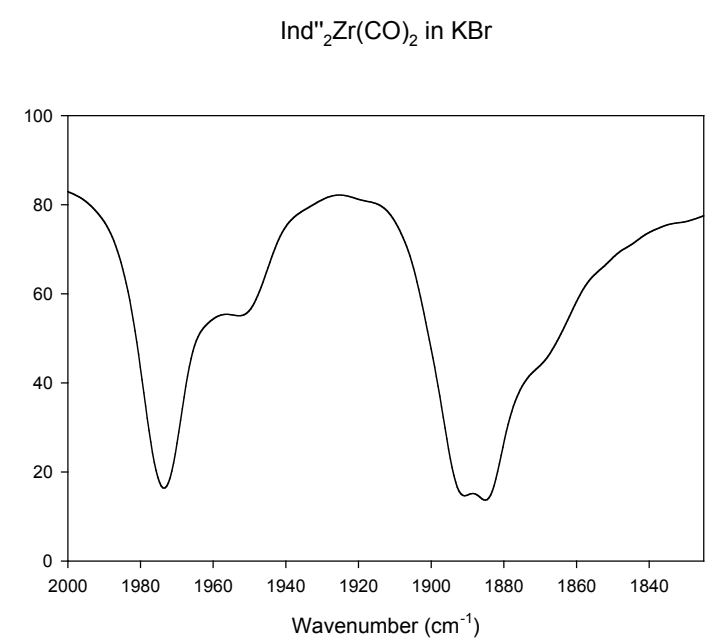

(c)

Ind"Ind" $\mathrm{Zr}(\mathrm{CO})_{2}$ in $\mathrm{KBr}$

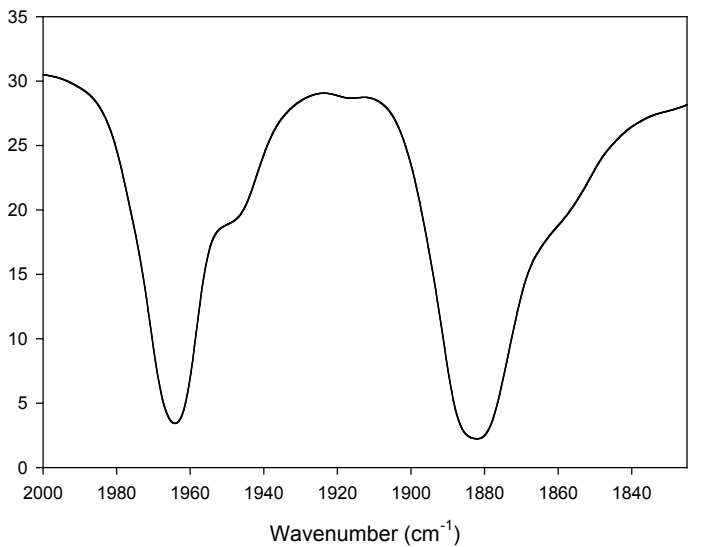

(b)

$\operatorname{lnd}^{\prime \#} \mathrm{Zr}(\mathrm{CO})_{2}$ in $\mathrm{KBr}$

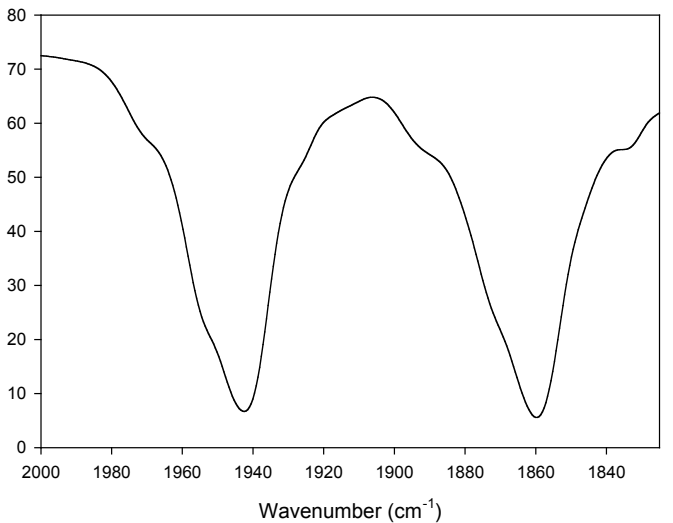

(d)

Ind $_{2} \mathrm{Zr}(\mathrm{CO})_{2}$ in $\mathrm{KBr}$

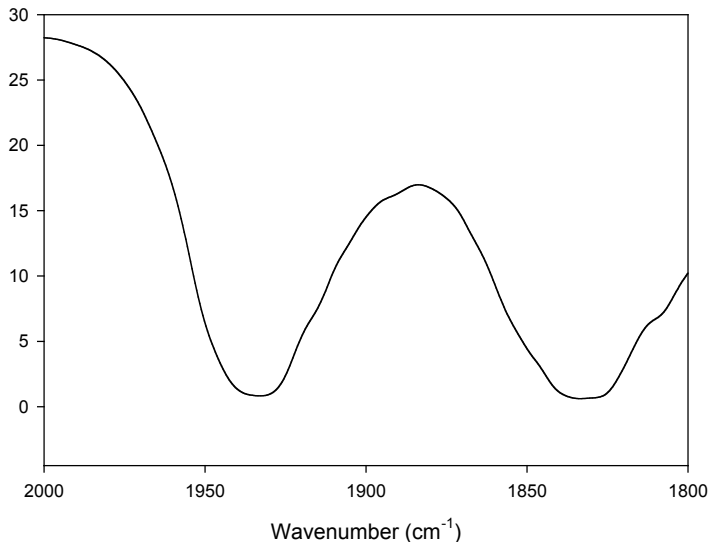

Figure S8. Carbonyl region of the infrared spectra of (a) 1-(CO) $)_{2}$ (b) 3-(CO) $)_{2}$ (c) 7-(CO) $)_{2}$ and (d) 5-(CO) recorded in $\mathrm{KBr}$. 


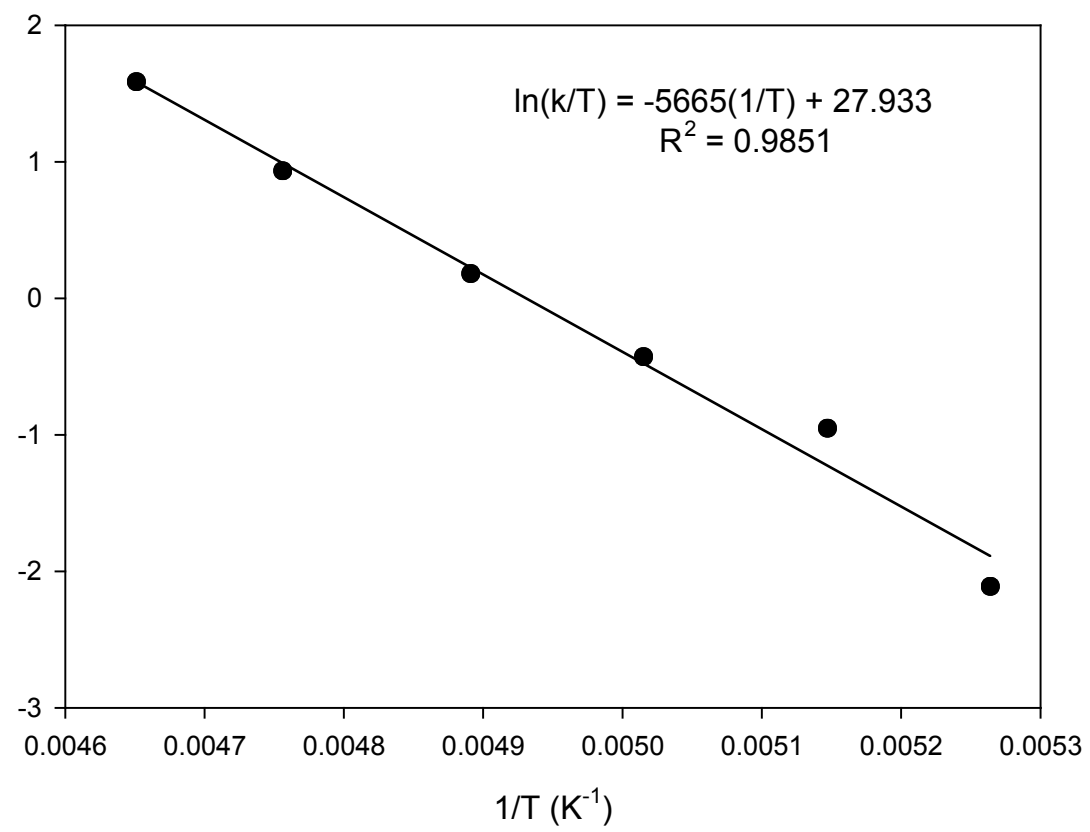

Figure S9. Eyring plot for $\mathbf{1 3}$ from variable temperature NMR data recorded in toluene- $d_{8}$. 


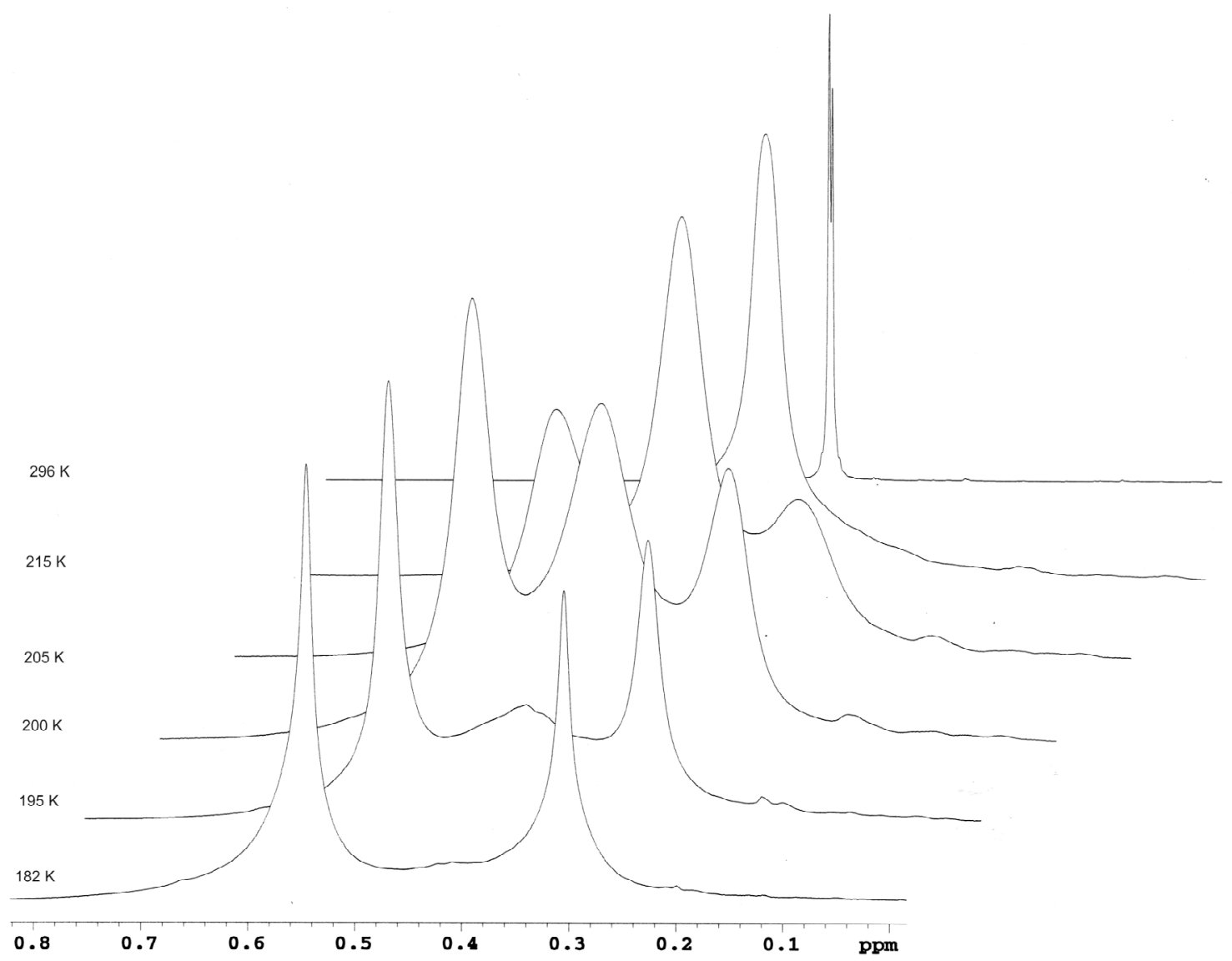

Figure S10. The trimethylsilyl region of the ${ }^{1} \mathrm{H}$ NMR spectrum of $\mathrm{rac} / \mathrm{meso}-\mathbf{1 7}$ in toluene- $d_{8}$ as a function of temperature. 


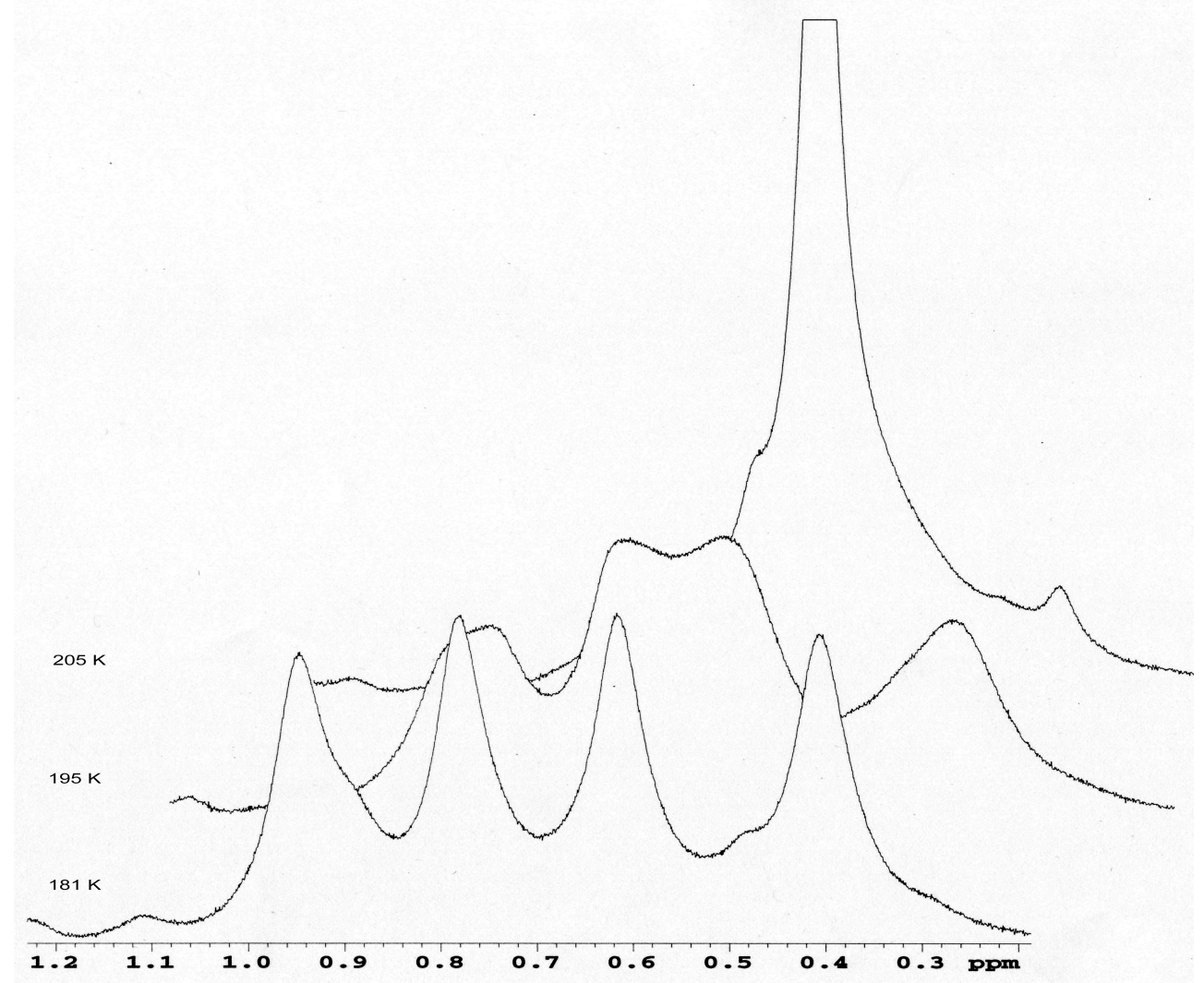

Figure S11. Silyl region of the ${ }^{1} \mathrm{H}$ NMR spectra of $\mathbf{1 4}$ in toluene- $d_{8}$ at low temperature. 